The ubiquity of mouthings in NGT

A corpus study 
Published by

LOT

phone: +31302536111

Trans 10

3512 JK Utrecht

e-mail: lot@uu.nl

The Netherlands

http://www.lotschool.nl

Cover illustration: (stemmed) word cloud of this dissertation, from tagul.com.

ISBN: 978-94-6093-158-1

NUR 616

Copyright (C) 2014: Richard Bank. All rights reserved. 


\title{
The ubiquity of mouthings in NGT A corpus study
}

\author{
Proefschrift \\ ter verkrijging van de graad van doctor \\ aan de Radboud Universiteit Nijmegen \\ op gezag van de rector magnificus prof. dr. Th. L. M. Engelen \\ volgens besluit van het college van decanen \\ in het openbaar te verdedigen op vrijdag 30 januari 2015 \\ om 12.30 uur precies \\ door
}

\section{Richard Bank}

Geboren op 1 augustus 1969

te Amsterdam 
Promotor: $\quad$ Prof. dr. Roeland van Hout

Copromotor: Dr. Onno Crasborn

Manuscriptcommissie:

Prof. dr. Pieter Muysken

Prof. dr. Jens Heßmann

(Hochschule Magdeburg-Stendal, Duitsland)

Prof. dr. Bencie Woll

(DCAL, University College London, Groot-Brittannië) 


\section{Table of contents}

Dankwoord ix

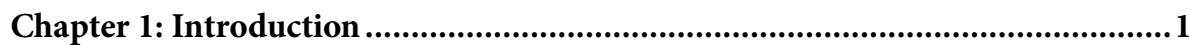

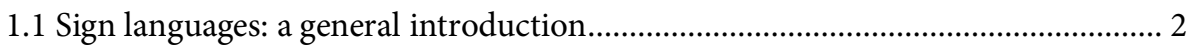

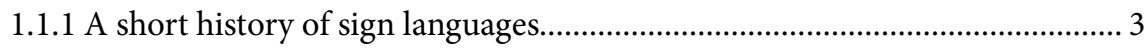

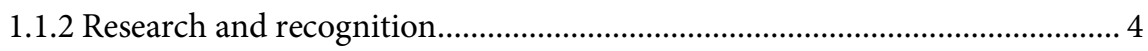

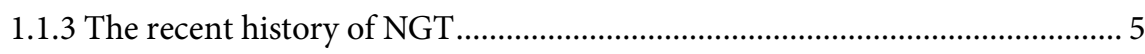

1.1.4 Deaf education in the Netherlands and influence of spoken language ........... 6

1.2. Mouth actions, Mouthings and Mouth Gestures ............................................... 8

1.2.1 Mouth actions, mouth gestures, mouthings ................................................ 9

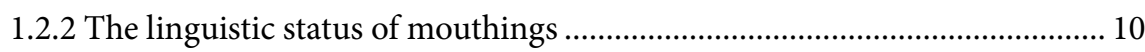

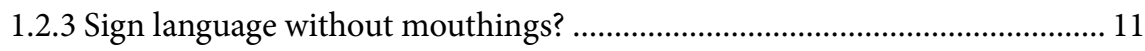

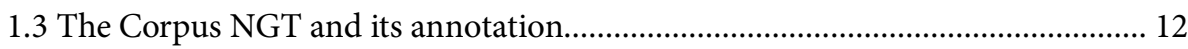

1.3.1 Written, spoken and signed language corpora ........................................... 13

1.3.2 Construction and contents of the Corpus NGT ........................................... 14

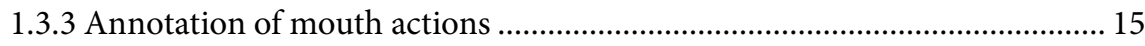

1.4 Research questions and outline of this thesis.................................................. 17

1.4.1 Variation in mouth actions with manual signs (Chapter 2) ......................... 18

1.4.2 The spreading of mouthings (Chapter 3).................................................. 18

1.4.3 The prominence of spoken language elements (Chapter 4) ........................ 19

1.4.4 Bimodal code-mixing (Chapter 5) ............................................................... 19

Chapter 2: Variation in mouth actions with manual signs ..............................21

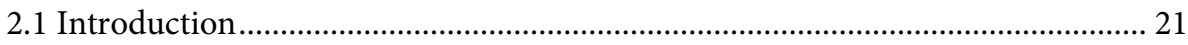

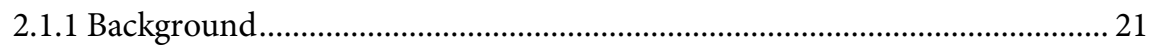

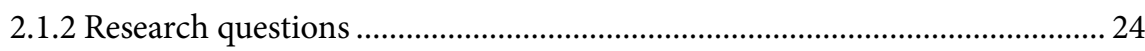




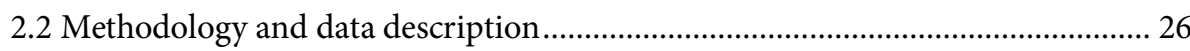

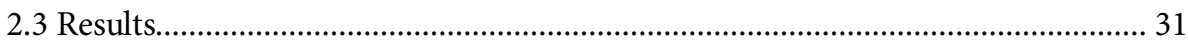

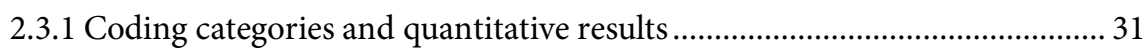

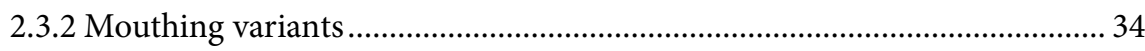

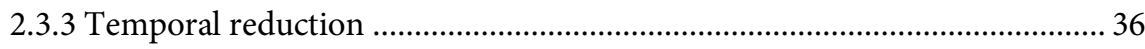

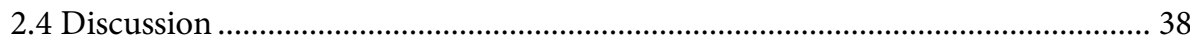

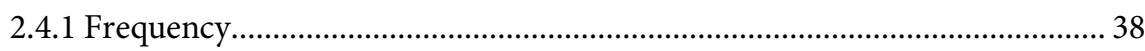

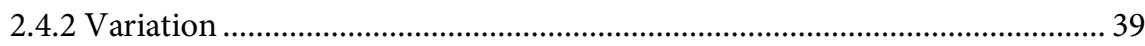

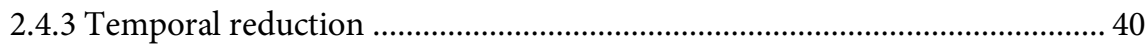

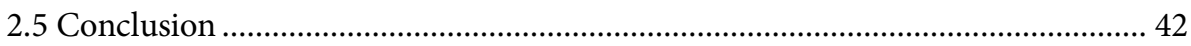

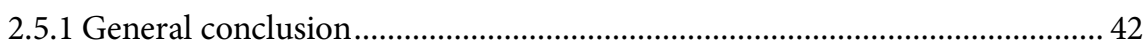

2.5.2 Concluding remarks on methodology ..................................................... 43

Chapter 3: Alignment of two languages: The spreading of mouthings ...............47

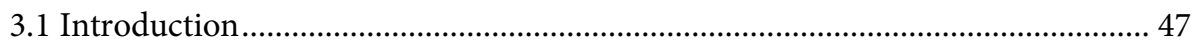

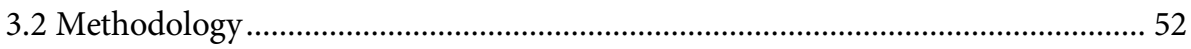

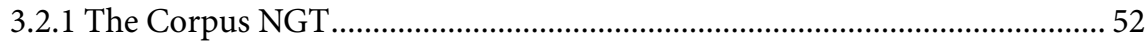

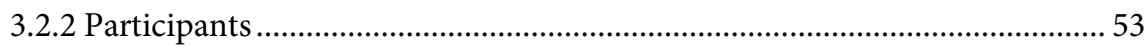

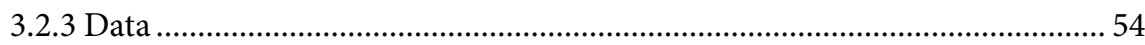

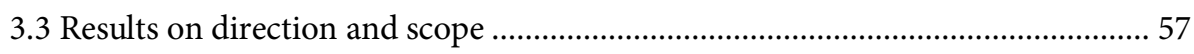

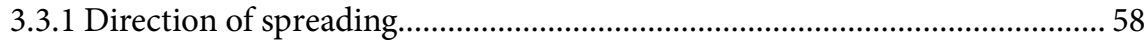

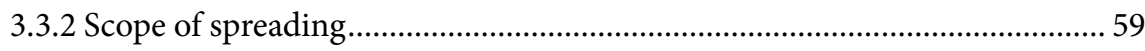

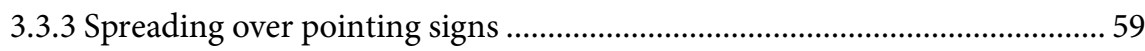

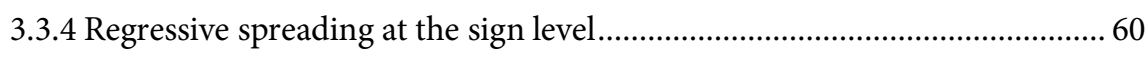

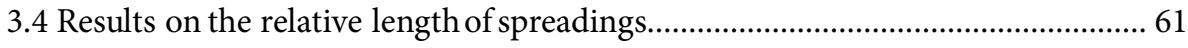

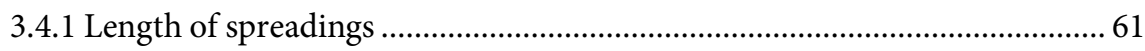

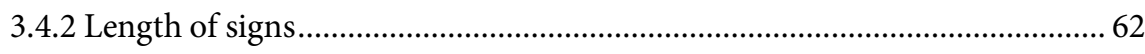

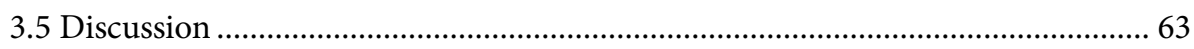

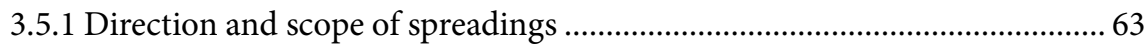

3.5.2 Spreading over PT and relative length of spreadings .................................. 64

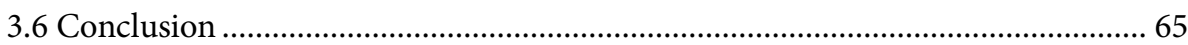


Chapter 4: The prominence of spoken language elements in a sign language .....67

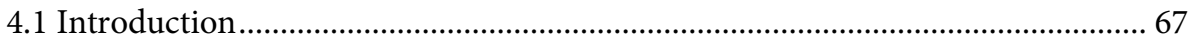

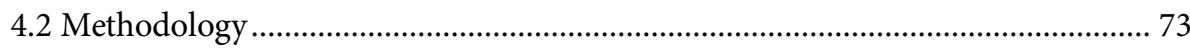

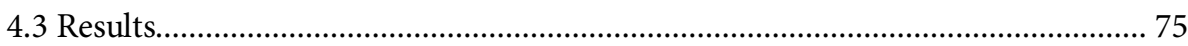

4.3.1 Results on mouth action frequency as compared to manual signs............... 75

4.3.2 Results on mouthing frequencies for various sociolinguistic variables........ 76

4.3.3 Results for combinations of mouth actions and pointings .......................... 78

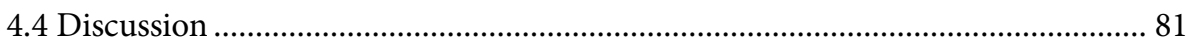

4.4.1 Proportion of mouth actions and mouthings compared to signs ................ 81

4.4.2 Mouthing frequencies related to various sociolinguistic variables............... 82

4.4.3 Combinations of mouth actions and pointing signs .................................... 83

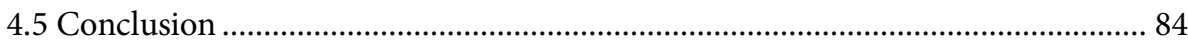

Chapter 5: Bimodal code-mixing: speech supported signing is the norm for deaf

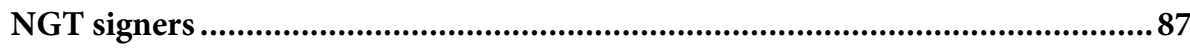

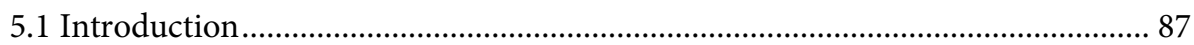

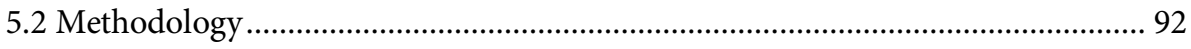

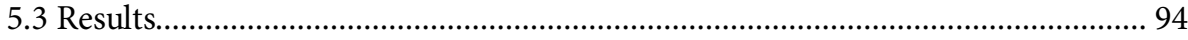

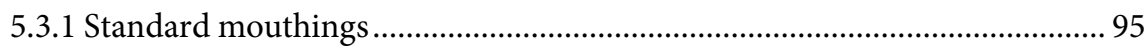

5.3.2 Solo mouthings used in backchanneling...................................................... 96

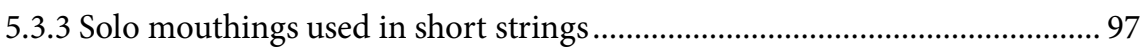

5.3.4 Specifying semantic information in content words (specifying mouthings) 99

5.3.5 Use of Dutch words in added mouthings .................................................. 101

5.3.6 Dutch word order and Dutch idiomatic expressions ................................. 107

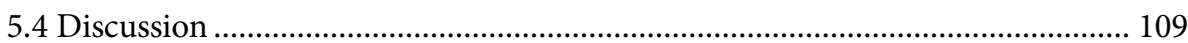

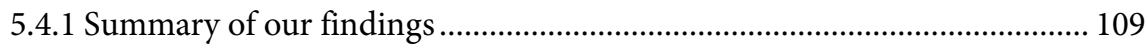

5.4.2 Implications for bimodal bilingual processing ........................................ 110

5.4.3 Implications for models of code mixing ...................................................... 111

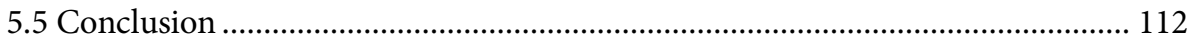

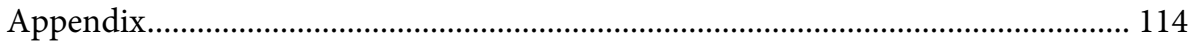


viii | Table of contents

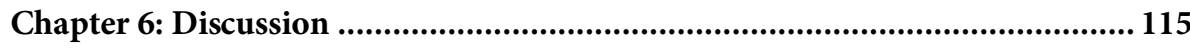

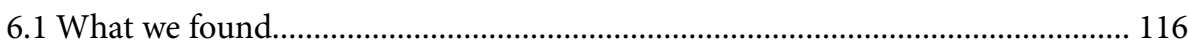

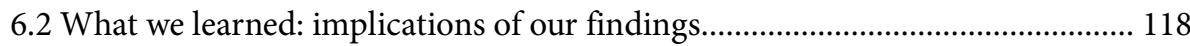

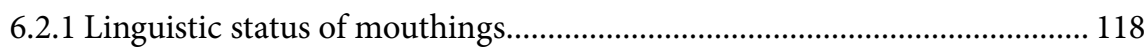

6.2.2 Code-mixing and code-blending .............................................................. 120

6.3 What we suggest for further research.......................................................... 121

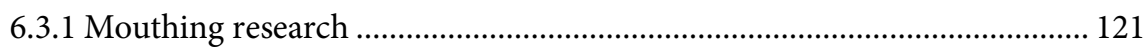

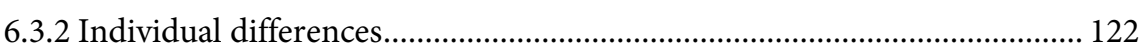

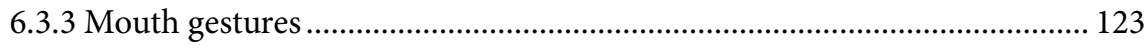

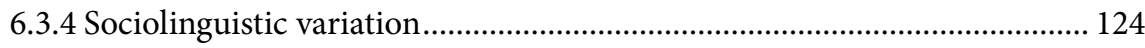

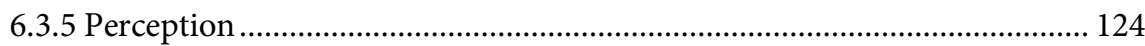

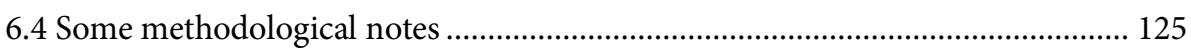

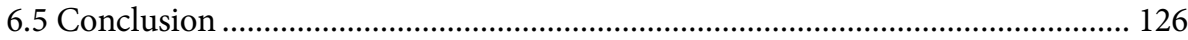

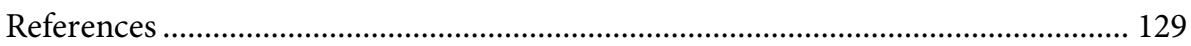

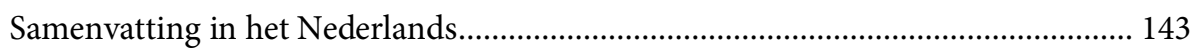

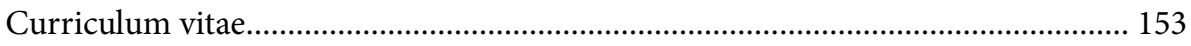




\section{Dankwoord}

(م) 20 maart 2009 kreeg ik van medestudent Adi Ben Arieh een e-mail met de vraag of het me nog gelukt was mijn dienst te ruilen in het hotel waar ik toen werkte, zodat ik meekon naar het concert van de Presidents of the United States of America, in Paradiso. En o ja, hij had nog wat gezien dat misschien wel interessant was voor mij: een promotieplaats over de rol van de mond in Nederlandse Gebarentaal. Dat was het begin van het project dat resulteerde in dit boek.

Hoewel mijn naam als enige op de kaft staat, zijn er vele mensen zonder wier betrokkenheid er helemaal geen boek was gekomen: bij discussies, besprekingen, koffiepauzes, lezingen, conferenties, afdelingsuitjes, cursussen, pizza-avonden en meer. Ik wil jullie hier graag bedanken.

In de eerste plaats mijn promotor Roeland van Hout en mijn co-promotor Onno Crasborn: jullie ondersteuning is van onschatbare waarde geweest, jullie zijn altijd positief gebleven en vol vertrouwen op een goede afloop, ook wanneer het soms wat minder ging. Roeland, je bent altijd erg betrokken geweest bij het project, iets wat ik altijd zeer gewaardeerd heb. Zelfs als je weg was was je nog bereikbaar, ik stelde me 
altijd voor dat je met de laatste versie van een of ander artikel onder je arm van de skihellingen afsuisde. Je wist ook altijd een oplossing te vinden voor alle statistiekvraagstukken - dank voor al je hulp. Onno, je bent een wandelende gebarentaalwetenschapskennisbank, en je stond altijd open voor vragen en suggesties, op wetenschappelijk vlak maar ook persoonlijk. Bovendien heb je je gerealiseerd hoe belangrijk een goede kop koffie is: je aanschaf van een Jura S7 zou voor in het handboek van iedere leidinggevende moeten staan. Ik vond (en vind) het een fijne samenwerking, bedankt daarvoor.

Ik ben blij dat ik in zo'n bijzondere en inspirerende onderzoeksomgeving heb mogen werken (en nog steeds werk). Hoewel mijn onderzoek een eenmansproject was heb ik me altijd een gewaardeerd lid van de onderzoeksgroep gevoeld. Dank daarvoor Anna (and for all the cat-sitting, too!), Anne, Ellen, Els, Inge (ook als paranimf!) en Martine; en aan mijn dove collega's: mijn gebaren waren misschien niet altijd al te best, maar het werken met jullie vond en vind ik inspirerend en leuk: dank jullie wel Johan, Merel (ook als paranimf!), Wim, Yassine en recenter ook Anique, Frouke en Max. Dank ook aan Asli, Beyza, Connie en Gerardo, mijn collega-gebarentaalonderzoekers bij het MPI. Emmy, Jacintha, Maria, Franziska en Shanley: bedankt voor het werk dat jullie voor jullie eigen projecten en scripties hebben gedaan en waar ik ook veel aan heb gehad. Han en Micha: bedankt voor alle ELAN-updates, -scripts en -bugfixes. Christel, Hella en Dirkje: dank voor alle ondersteuning. Cefas, Eric, Eva, Job, Louis, Maarten, Mario, Odette, Remy en vele anderen: dank voor alle taart- en koffieleut, en Vanja ook als kattenoppas! Stephen: thanks for being my in-house native speaker of English whenever I needed one. Thanks also to my fellow IMPRS students, and Rachel, Dirkje and Els: I may not have spent much time with you, but the meetings we had were fruitful and I learned from you.

Inge, ik had me geen fijnere kamergenoot kunnen wensen. Je voelde haarfijn aan als je me 's ochtends met rust moest laten als de NS weer eens naar deed. We spraken elkaar moed in wanneer dat nodig was, of hadden het over dans, muziek, THHGTTG of gebarentaal wanneer dat kon. Ik vind het jammer dat je zo ver weg woont.

Een bijzonder woord van dank ook aan de participanten van het Corpus NGT. Jullie hebben je laten filmen ten behoeve van wetenschappelijk onderzoek, zonder precies te weten wie er waarom op welke manier naar zou gaan kijken. Ik heb uren besteed aan het analyseren en beschrijven wat jullie nu precies zeggen, en voor mijn gevoel heb ik sommigen best goed leren kennen. Wellicht wordt dat ooit wederzijds. 
Vier dagen per week heen en weer reizen van Amsterdam naar Nijmegen is een paar jaar goed gegaan, maar ik was blij dat ik het laatste jaar voor twee dagen per week aan een bureau in het Bungehuis in Amsterdam kon aanschuiven. Daar heb ik me altijd heel welkom gevoeld; bedankt hiervoor Marijke en Joni, maar ook Roland, Vadim, Elly en Gerdien. Het was sowieso leuk mijn vroegere studiegenoten en docenten daar weer te zien en nieuwe mensen te leren kennen, zoals op de vrijdagmiddagborrels, waarbij ik wat langer kon blijven plakken dan ik in Nijmegen zou doen en toch nog op tijd thuis kon zijn - dank Jan-Willem, Sophie, Karin, Sterre, Iris, Klaas, Margot, Jan, Paul, Rob, Ingrid en alle anderen.

En over borrels gesproken: als er de maandelijkse quizzen en andere feestelijkheden er niet waren geweest, had ik helemaal geen sociaal leven meer gehad. Dank voor mijn geestelijke gezondheid Andries, Astrid, Barbara, Carolien, Ed en Margot, Hans en Nina, Henderina, Jacq, Jan, Janneke, Maarten en Ellen, Majel, Mariska en Sander, Lianne, Mettine, Michiel en ook iedereen die ik hier niet bij naam genoemd heb.

Aan mijn lieve familie en schoonfamilie: dit is waarom jullie me bijna nooit meer zagen. Ik hoop dat ik de tijd nog in kan halen. Pa en ma, Arthur en Sandra, Corrie, Sjouke en Anneke, en Dik: dank dat jullie er zijn.

Maar uiteindelijk is er maar één zonder wie er niet eens een begin was gemaakt met dit project, laat staan dat het tot een goed einde gebracht had kunnen worden: Sas, dank voor je steun, voor je liefde, voor je vertrouwen, voor alles. 
xii | 


\section{Chapter 1: Introduction}

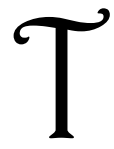

he aim of this thesis is to deepen our understanding of how Dutch spoken language combines with everyday signing in Sign Language of the Netherlands

(NGT, Nederlandse Gebarentaal). NGT is the language of the Dutch deaf community, and is used as the first and preferred language by an estimated 7,500 (Wheatley \& Pabsch, 2012) to 30,000 (Wheatley \& Pabsch, 2010) deaf signers, and by several thousands of hearing people as a second language (such as hearing children of deaf adults or sign interpreters). Sign languages are often thought of as manual languages, but it has been demonstrated for all sign languages that facial expressions and head and torso movements also form part of the grammar and use of sign languages (Baker-Shenk, 1983; see Crasborn, 2006, for an overview). Moreover, when deaf NGT signers sign among themselves, their mouths are constantly moving as well. Some of these mouth actions are sign language inherent, but one can also observe many Dutch lexical items. This thesis will explore the way Dutch lexical items are used by different cross-sections of deaf native signers, how these items vary between tokens of the same sign, and how they combine with signs and sentences. In addition to the 
studies in this thesis, the data gathered for these studies was published in the second release of the Corpus NGT annotations, in the autumn of 2014 (see www.ru.nl/corpusngtuk).

This introductory chapter starts with a short overview of the history of sign languages and sign language research, with a focus on the education of the deaf in the Netherlands (1.1). We will then describe and define mouth actions and particularly mouthings, the focus of this thesis (1.2). Next, we describe the Corpus NGT which provided the raw data analysed and reported in this thesis, and we will compare this corpus to other sign language corpora (1.3). Finally, the research questions will be formulated, followed by an outline of the chapters of this thesis (1.4).

\subsection{Sign languages: a general introduction}

In deaf communities around the world, sign languages are the principal means of communication. The number of sign languages in use today is unknown, however. Woll, Sutton-Spence, and Elton (2001) discuss the difficulties in counting the number of sign languages, and consequently do not give an estimate. For example, nomenclature is a problem: sign languages do not have distinct names other than the labels linguists have given them. Another problem is the relation between varieties: is there one common variety, or are there varieties that differ enough to justify different language labels? These issues are familiar from the spoken language domain (Makoni \& Pennycook, 2005, 2006). The index of Sign Language: An International Handbook (Pfau, Steinbach, \& Woll, 2012) lists 93 different sign languages. Some of those have become extinct (e.g. Martha's Vineyard Sign Language, see Groce, 1985), others were used by hearing communities under a vow of silence (e.g. Cistercian Sign Language) or were used to work in a noisy environment (e.g. Saw Mill Sign Language; Pfau, 2012). The ethnologue website (www.ethnologue.com) lists 137 sign languages, counting only those used as primary languages by deaf communities. Meier (2000) has suggested there may be as many as 200-300 sign languages. The actual number is likely to be much higher, given the limited research so far in large parts of the world such as in China and India.

In this section we will briefly sketch the emergence of sign languages (1.1.1) and sign language research (1.1.2), and then describe the state of affairs for the deaf in the Netherlands (1.1.3). 


\subsubsection{A short history of sign languages}

It has been suggested that the first traces of language used by early humanoids may have been signed and not spoken (e.g. Armstrong, 1999; Armstrong \& Wilcox, 2007). The use and existence of signed communication has been discussed as early as 500 BCE in Greece by Socrates (in Plato's Cratylus), it is laid down in the Mishnah (a written version of Jewish oral law) around the second century CE (Danby, 1933: 313), and it was valued and recognised at the Turkish Ottoman court from the $15^{\text {th }}$ century on (see also McBurney, 2012). One of the most important - and best documented events in the recent history of western sign languages took place in the second half of the $18^{\text {th }}$ century, when the Parisian abbé Charles-Michel de l'Épée founded the first public school for deaf children (Lane, 1984). His goal was to educate deaf people through the sign language they were already using, mixing it with invented gestures in order to express specific aspects of French grammar. By bringing groups of deaf people together, the signs that these people were already using began to transform into a viable, full-fledged sign language. The method of de l'Épée proved to be successful and schools for deaf children were founded all over France. His manual method spread rapidly through the rest of Europe and the United States of America. Around the same time as de l'Épée, other initiatives for deaf education were taken in Europe, for instance by Samuel Heinicke in Leipzig and Thomas Braidwood in Scotland (McBurney, 2012). While the exact influences of the different teaching methods on the advance of the various sign languages remain unclear, the foundation of schools for the deaf (and consequently the gathering of deaf people) certainly helped sign languages evolve. A contemporary example of this scenario can be seen in Nicaragua, where a deaf community was created with the start of deaf education in the late 1970s. (Adolescent) children from various parts of the country were brought together, bringing in their home signs - idiosyncratic gesture systems that develop in the absence of a language model (Mylander \& Goldin-Meadow, 1991). In their new school, these children were not taught sign language, but within a few years their mutual communication began to display aspects of a real language, and Nicaraguan Sign Language was starting to emerge (Kegl, Senghas, \& Coppola, 1999; Senghas, 1995).

In the second half of the $19^{\text {th }}$ century, oralist methods were gaining ground in deaf education. This culminated in 1880 in Milan at the International Congress on the Education of the Deaf, where it was decided that the method of oral teaching was to be preferred over using methods drawing on signing. Consequently, schools for the 


\section{4 | Chapter 1: Introduction}

deaf abandoned signing, complicating students' communication and education (Lane, 1984), also in the Netherlands (Schermer, 1990). This resulted in spoken language education for people whose most accessible language was a visual one, a situation that lasted until 1980, two decades after the onset of the scientific recognition of sign languages as full-fledged languages.

\subsubsection{Research and recognition}

The language of gestures, expression, impersonation, pantomime or acting is the fundamentally natural, universal and international language. We used it as babes, our parents used it to teach us and to correct us during the first years of our life. Even now we revert to this language to make the meaning of our words clearer and more emphatic. [...] Many persons consider this language to be proper only to the deaf, but they err. This language of gestures is used in many occupations of life. (Higgins, 1923[1959: 2])

Although Higgins stands up for the use of sign language, and speaks against the use of the term 'deaf-and-dumb', the quotation above makes clear that he does not separate signing from co-speech gesturing. At the time, sign language research was nonexistent. One of the first forays into the study of sign language structure was Tervoort's (1953) dissertation. From the observation that Dutch deaf children's communication with hearing people was strenuous and difficult, yet communication among themselves seemed effortless, Tervoort hypothesised that these children might be using a language with a structure different from spoken Dutch. Five girls between twelve and fourteen years old were tested, and the language skills of twenty others were investigated. Tervoort induces that the visual language and the spoken language are essentially different, but that a visual cue may be a word, a sign or a combination of the two (see section 1.2, below). One of Tervoort's main conclusions is that

The behaviour of the children is linguistic: hence we may speak of an esoteric language. Signs are used in various contexts, but they are identical in form and meaning while variously applied. There is a tendency towards morphological and syntactical categorizing: this tendency is still in a rudimentary state and is overlaid with attempts at forming categories which have no linguistic motivation. (Tervoort, 1953: 294). 
McBurney (2012), however, argues that "the signing he [=Tervoort] studied was not a complete and natural sign language. The Dutch educational system forbade the use of signs in the classroom, so most of the signs the children used were either home signs or signs developed amongst the children themselves" (2012: 925). Either way, another study, one that sparked the greater research into sign language, was Stokoe's (1960[2005]) analysis. Triggered by the observation that signs might be analysed along the lines of minimal pairs, Stokoe, with the help of two research assistants, conducted a "repeated study of some five thousand feet of film taken at normal and slow motion speeds of the signing of fourteen deaf and two hearing informants" (1960[2005: 16]). Stokoe devised the first notation system for signs (which came to be known as Stokoe notation), and showed that signs have an internal structure, that is, a phonology: every sign has a location, a movement and a handshape.

Stokoe's work may have been influential, but the book work that definitely marked the acceptance of sign language as a full-fledged language was Klima and Bellugi's The Signs of Language (1979). The book describes a series of studies and experiments that cover various topics such as a feature analysis of handshapes (organising handshapes in a way analogue to speech sounds), the grammatical use of space (describing the internal systematicity of inflection), slips of the hand (establishing the psychological reality of the various parameters of signs), and a crosslinguistic comparison of American Sign Language (ASL) and Chinese Sign Language (demonstrating that sign languages have formational constraints specific to that language), among others.

\subsubsection{The recent history of NGT}

While ASL was being explored and described, there was hardly any research on sign language in the Netherlands in the decades since 1953, and signing did not yet have the label NGT. Tervoort (1953) called the language among deaf children esoteric, as opposed to the exoteric language that deaf children used with hearing interlocutors. Development of a notation system for signs (KOMVA, 1988) was started in 1982 together with the compilation of a dictionary (KOMVA, 1989) - showing that research interest began to increase. It is telling that Schermer (1990) titled her dissertation In search of a language. In her book, she indeed assumes the existence of a full-fledged Sign Language of the Netherlands, whether or not influenced by Dutch spoken language. Her findings corroborate her assumption, as "the communication system of the deaf participants in our study can be described as a system that involves 
sign language and shows some influence of spoken Dutch" (Schermer, 1990: 142). Since then, many aspects of NGT have been studied, such as the use of signing space by children (Knoors, 1992), the occurrence of agreement markers (Bos, 1993), first language acquisition (Van den Bogaerde, 2000), and its phonology (Crasborn, 2001; Van der Kooij, 2002). This has contributed to the 'establishment' of NGT, an important factor in the emancipation of the deaf in the Netherlands.

\subsubsection{Deaf education in the Netherlands and influence of spoken language}

The first institute for education of the deaf that was established in the Netherlands was that of H.D. Guyot in 1790, in the northern Dutch city of Groningen. Guyot was a Protestant pastor with an interest in teaching deaf children. Having heard of the work of abbé de l'Épée, he went to Paris to learn de l'Épée's methods, which he incorporated into his own teachings. Thus, the H.D. Guyot School became an institute where the acquisition of both signed and spoken language was important. Fifty years later, in 1840 , a second institute was established in Sint-Michielsgestel, a town in the south of the Netherlands. The principal teacher at this Instituut voor Doven (Institute for the Deaf) was Martinus Verbeek. Verbeek based his method on de l'Épée's, but designed his own signing system, where syntax and grammar followed Dutch and lots of signs were initialised (Derks, 2004). Both the Groningen and Sint-Michielsgestel schools were boarding schools. The third institute was established in 1853 in Rotterdam, and was strictly oralist. Pupils were not housed on the school premises, but accommodated at foster homes. In 1888, the oralist Effatha was established in Leiden (and later moved to Dordrecht and then Voorburg), followed by the oralist Ammanschool in Amsterdam, in 1911. Table 1.1 provides an overview (based on Van Veen, 2012). 
Table 1.1. Overview of the schools for the deaf in the Netherlands (based on Van Veen, 2012).

\begin{tabular}{lllll}
\hline \hline School name & Location & Est. & Internal/external & Origins \\
\hline $\begin{array}{l}\text { H.D. Guyot } \\
\text { Instituut voor } \\
\quad \text { Doven }\end{array}$ & $\begin{array}{c}\text { Groningen } \\
\text { Sint-Michiels- } \\
\text { gestel }\end{array}$ & 1790 & Boarding school & Signing \\
RMI & Rotterdam & 1828 & Boarding school & Signing \\
Effatha & Leiden/Voorburg & 1888 & Foster homes & Oralist \\
Ammanschool & Amsterdam & 1911 & Foster homes & Oralist \\
\hline \hline
\end{tabular}

Prior to the 1880 Milan conference, where oralism was declared to be the standard in deaf education, the Guyot school had already abolished signing in 1864, except for the children who were unsuccessful in the oralist method (Kolen, 2009; Van Veen, 2012). The Instituut voor Doven in Sint-Michielsgestel followed in 1906. From then on until the 1980s, all deaf education in the Netherlands was oralist.

Beginning in 1980, schools have started to open their minds to the idea that mastery of sign language could not only be a means to an end, but also a goal in itself (Lane, 1984). In the Netherlands, the peak of this development was reached with the start of bilingual deaf education throughout the country in 1997.

Until about 1990, there had been five institutes in the Netherlands that educated deaf children. Since then, the number of schools has been increasing while the overarching institutes have kept merging so that there are now only two large organisations (Kentalis and Auris). At the deaf schools, there is no monolingual language input; both sign and spoken language are important. The number of deaf staff at the schools is limited, however, and the NGT skills of the hearing staff are not always optimal. Moreover, the majority of young pupils entering the schools for the deaf have been implanted with cochlear implants (CI), enabling them rudimentary sound perception and speech comprehension. Actual numbers of implanted children are hard to come by, but estimations about the percentages in the Netherlands range from 70-75\% (De Raeve et al., 2009) to over 90\% (Knoors \& Marschark, 2012). Cochlear implants are the norm nowadays, and although they seem to have been accepted by the deaf community as an acceptable type of hearing aid, there is a general concern in the deaf world about the potential impact this has on the viability of deaf 
culture and sign language. Most implanted deaf children now move on to mainstream (hearing) schools, where they will have little to no sign language input, threatening the sustainability of a deaf culture with its own language. On the other hand, the rise of easy to use online video sharing platforms, combined with cheap and omnipresent video recording possibilities through smartphones and webcams, creates unimagined opportunities for deaf signers to interact in their native language (and globally through international sign; see Hiddinga \& Crasborn, 2011).

\subsection{Mouth actions, Mouthings and Mouth Gestures}

Modern day sign language, thus, has had a lot of oralist influence in the Netherlands. Tervoort (1953) already noted in his dissertation that hand and mouth often act together:

Word and sign, mouth movement and hand movement, are parts of one token, but a complex token: this is evident from the fact that they can occur independently of each other and denominate independently in an analogue way. If both parts appear in unity then they undergo each other's influence.

Let us first consider mouth movement. Its form is influenced by the hand movement. Its realisation does not need to have the visual clarity of the independently used visual word, since the sign also denominates and its form particularly aims at providing clarity. An often carelessly realised articulation is the result. [...] As a consequence of the binding to the sign, the choice of words is limited. A fixed sign naturally has the use of one word that belongs to it as a result. (Tervoort, 1953: 109-110; translation mine)

Apart from Tervoort's observation quoted here, mouth actions have been largely neglected in sign language research until quite recent times. We will first discuss the most important studies on mouthings and mouth gestures (1.2.1), continue with discussing the linguistic status of mouthings (1.2.2) and end up with the question whether a hypothetical 'pure' sign language - without mouthings - is possible or not (1.2.3). 


\subsubsection{Mouth actions, mouth gestures, mouthings}

A 1998 workshop in Leiden, the Netherlands, was the basis for a book on mouth configuration and movement: The hands are the head of the mouth, edited by Boyes Braem and Sutton-Spence (2001). The book assembled papers on mouth action research in various sign languages from various theoretical points of view. One of the aims of the workshop was to standardise terminology for two broader types of mouth patterns. First, there are mouth patterns that are clearly derived from spoken language, articulated together with a sign. These had been termed 'spoken components', 'word pictures' and 'mouthings'. Mouth patterns that were not derived from spoken language had been termed 'oral components', 'oral adverbials', 'mouth arrangements' and 'mouth gestures' (Boyes Braem \& Sutton-Spence, 2001). Although no consensus was reached at the workshop, the terms 'mouthings' and 'mouth gestures' are used rather consequently throughout the book, resulting in a de facto standardisation of the terms in later research.

The use of mouthings is reported for all nine sign languages discussed in the book: eight European sign languages (British, Finnish, German, Italian, Norwegian, Swedish, Swiss-German Sign Languages, and NGT) and one non-European sign language (Indopakistani Sign Language); most data come from small corpora or introspection.

After Vogt-Svendsen's $(1981,1983,1984)$ pioneering studies on Norwegian Sign Language, one of the first in-depth descriptions of the co-occurring activity of the hands and the mouth is Schermer's (1990) dissertation on NGT. Schermer analysed the signing of six informants who retold a written story and a story from a picture book, and who, in addition, were engaged in a short stretch of spontaneous conversation. She describes both form and function of mouthings ('spoken components', in her words), and places them in one of three categories: signs without a mouthing (including signs with a mouth gesture), mouthings without a sign, and combinations of signs and mouthings. The latter category has three subcategories: redundant mouthings (semantically corresponding with the manual sign they accompany) that consist of full Dutch lexical items, redundant mouthings that consist of parts of Dutch lexical items, and functional mouthings. The latter category is split into three different functions. First, mouthings can disambiguate the sign they accompany. One example is the mouthing wetenschap ('science') with the sign TO-KNOW, to disambiguate from TO-KNOW with weten ('to know'). Second, mouthings can complement the sign they accompany, to narrow down a meaning. 
Examples are HAIR with the mouthing blond ('blond') for 'blond hair', or ARRIVE with the mouthing thuis ('home') for 'arrive at home'. Third, mouthings can specify the sign they accompany by indicating time and/or person. An example is TO-COME with the mouthing kwam ('came'), to specify the sign for past tense, singular (all examples from Schermer, 1990: 125-127). The majority of mouthings, however, fall in the 'redundant' categories. Schermer notes that the fact that a redundant mouthing

does not seem to fulfil a specific function in the language, does not rule out that the articulatory movements and the lip/mouth pictures are not necessary for the deaf signer. That is why the term 'redundant' should not be interpreted as 'superfluous' (Schermer, 1990: 137)

Studies on other sign languages suggested comparable functionalities (cf. Boyes Braem, 2001, for Swiss-German Sign Language; Crasborn, Van der Kooij, Waters, Woll, \& Mesch, 2008, for British and Swedish Sign Languages; Fontana, 2008, for Italian Sign Language; Hohenberger \& Happ, 2001, for German Sign Language; Mohr, 2011, for Irish Sign Language; Vogt-Svendsen, 1984, 2001, for Norwegian Sign Language). There are, however, no processing studies that investigate the hypothesis implicit in Schermer's statement above, that perceivers indeed make use of the lexical information articulated by signers on the mouth.

\subsubsection{The linguistic status of mouthings}

While it is generally accepted that mouthings originated as borrowings from spoken languages, there is an ongoing debate about their linguistic status (papers in Boyes Braem \& Sutton-Spence, 2001). Several researchers analyse mouthings as part of sign language structure (e.g. Ajello, Mazzoni, \& Nicolai, 2001; Bergman \& Wallin, 2001; Boyes Braem, 2001; Sutton-Spence \& Day, 2001; Vogt-Svendsen, 2001). In this view, mouthings form part of the sign language lexicon and are integrated into the morphosyntactic structures of sign languages. Other researchers explain the occurrence of mouthings in their data purely as a language contact phenomenon and thus coincidental to sign languages. Zeshan (2001), for instance, in a description of mouthing use in Indopakistani Sign Language (IPSL), argues that "the extremely variable amount of mouthing observed across individuals is $[\ldots]$ naturally explained by the extremely diverse linguistic and personal backgrounds of IPSL-users" (2001: 251). Likewise, Hohenberger and Happ (2001), in an account of mouthing use 
in German Sign Language, argue that the origin of mouthings is oralist education. They claim that mouthings are only a performance phenomenon and not a competence phenomenon, thus relating to language use and not the structure of language including knowledge of the lexicon. They further argue that sign languages can dispense with mouthings, and that this view is supported by the varying degrees of mouthings observed in different signers.

Contact between spoken and signed language, be it in an educational setting or in contact among signers from different spoken language backgrounds, thus leads to the use of spoken language elements in signed language, or code-mixing. As will also be explained in the following chapters, the notion of code-mixing is problematic for sign languages, as it is usually understood as a unimodal, sequential phenomenon (cf. Muysken, 2000). The term code-blending, coined by Emmorey, Borinstein, and Thompson (2005; see also Emmorey, Borinstein, Thompson, \& Gollan, 2008), has been gaining ground recently as a way of describing bimodal simultaneous use of two languages, similar to code-mixing between spoken languages.

An alternative view on the linguistic status of mouthings is put forward by Ebbinghaus and Heßmann (2001). They reject the idea that mouthings are a form of code-mixing, because that presupposes a pure, unspoilt state of the sign language without influences from surrounding spoken languages: "there is neither diachronic nor synchronic evidence for the existence of such a 'wordless' sign language. Rather, all the evidence there is points to the following conclusion: words are neither mixed in, nor switched to or borrowed, but, quite simply, used in a sign language context" (2001: 139). In their view, sign language is a form of multidimensional communication, where mouthings are just one of the three components, together with manual signs and non-manual signals. All three components are important in communication, resulting in what Enfield (2009) terms 'composite utterances'.

\subsubsection{Sign language without mouthings?}

A sign language that has long been claimed to be virtually devoid of mouthings is ASL. Of course, American deaf signers do come into contact with their surrounding spoken language, just like European signers do with their respective spoken languages, but the effects of this contact surfaces in ASL more as fingerspelling than as mouthings (Boyes Braem, 2001). As it turns out, however, the main reason for the lack-ofmouthings claim turns out to be lack of research: quite recently, Nadolske and Rosenstock (2007) showed that ASL signers frequently do use mouthings. It raises again the question whether there is a 'pure' sign language; deaf signers live in a hearing 
world, since there is no deaf country where signed language suffices for all communication. Indeed, as most deaf children are born to hearing parents, spoken language input is dominant in the early life stages of the large majority of deaf children.

As will become clear in the following chapters, all signers recorded in the Corpus NGT use mouthings, even though there were no hearing people around during the recording session (see further section 1.3.2, below). Earlier, Schermer (1985) noted that "the existence of a pure sign language, without the occurrence of any speech, among deaf adults, is more or less a theoretical construct" (1985: 288). Almost thirty years later, this still seems to hold. As far as we know, there is only one sign language where mouthings are rarely used by its signers: Kata Kolok in Indonesia, a rural sign language in which "there are virtually no mouthings" (De Vos \& Zeshan, 2012: 17). Most signers of Kata Kolok are hearing - not unlike the situation in Martha's Vineyard (Groce, 1985), where the number of deaf people was relatively high and sign language was also used by a large number of the hearing people in the community; while studies on Kata Kolok usually involve deaf signers, it is unclear whether hearing Kata Kolok signers would use more mouthings. In other communities with a high incidence of hereditary deafness, the use of mouthings with sign language is more common. Nyst (2007), for instance, reports that $15 \%$ of the signs in her Adamorobe Sign Language database come with mouthings.

\subsection{The Corpus NGT and its annotation}

Many studies on sign language involve observations of small data sets of small numbers of deaf signers, or introspection of hearing signing researchers. While these studies have been relevant and important, they may offer a non-representative view of sign language as it is actually used by its signers. To systematically study (sign) language use in settings as natural as possible, one needs to make use of an annotated corpus. In the case of signed language, this involves video recording as a first step, followed by transcription and annotation. This section will describe the Corpus NGT in the context of spoken and signed language corpora (1.3.1), give a more detailed view on how the Corpus NGT came to be and what it looks like (1.3.2) and conclude with a description of mouth action annotations (1.3.3). 


\subsubsection{Written, spoken and signed language corpora}

Sign language corpora are a recent phenomenon in corpus linguistics. The earliest corpora were written concordances of works like the Bible or Shakespeare's work not made for linguistic research but for a better understanding of the work in question. The first computer generated concordances used punch-cards for storage, and brought the time to process some 60,000 words down to 24 hours (McCarthy \& O'Keeffe, 2010). Advances in computer technology resulted in large databases that could be searched through a few clicks of the mouse, such as the CHILDES database for child language research, released in 1984 (MacWhinney, 2000). Later, the growing processing power and storage capacity of computers made it possible to construct corpora containing audio and video recordings.

Spoken language corpora can be annotated (and are easily searchable) by virtue of the existence and use of orthographies and phonetic transcription systems. While several writing systems were developed for sign languages (see Frishberg, Hoiting, \& Slobin, 2012, for an overview), none of them is generally accepted, and it would require a lot of training to master one. Annotating sign language videos using glosses (representing the signs in another language) is much easier and makes the possibilities for corpus research much more versatile, despite disadvantages such as the interference of the semantics of the spoken (written) language in the glosses (Johnston, 2008, 2010). However, there are currently no straightforward solutions that produce reliable automatic sign language notations or glosses.

Sign language corpora are therefore painstakingly and laboriously coded by hand. As a consequence, only small parts of the existing sign language corpora have been glossed and annotated. The number of sign language corpora is growing; among the most elaborate ones are the Auslan corpus (e.g. Johnston, 2008, 2013), the British Sign Language corpus (Schembri, 2008; Schembri, Fenlon, Rentelis, Reynolds, \& Cormier, 2013) and the Corpus NGT (Crasborn, Zwitserlood, \& Ros, 2008; Crasborn \& Zwitserlood, 2008b; see further section 1.3.2, below). These corpora are among the bigger ones, and they are available online. Many more corpora are currently under development, especially in Europe (such as in Belgium, Germany, Poland, Slovenia, and Sweden, to name just a few). The way corpus annotations are organised is motivated by the research questions that underlie the corpus' construction. In the Corpus NGT, for instance, information on the relation between left- and right-hand glosses was sacrificed for the benefit of research on the apparent independence of the two manual articulators (see Sáfár \& Crasborn, 2013). Although 
several corpus projects have issued guidelines for annotation (e.g. Crasborn, Mesch, Waters, Nonhebel, Van der Kooij, Woll, \& Bergman, 2007; Johnston, 2010), there is no general agreement on annotation standards. Recent arguments for standardising sign language corpus annotation have been made by Johnston (2008), and Schembri and Crasborn (2010).

\subsubsection{Construction and contents of the Corpus NGT}

In the studies reported in the next four chapters, we used parts of the Corpus NGT as our dataset. The Corpus NGT was constructed from 2006 to 2008, and contains video data of 92 prelingually deaf signers, recorded in pairs. Participants were matched in generation and regional background; data are available from all five regions in the Netherlands where deaf schools are located (Amsterdam, Groningen, Rotterdam, Voorburg and Sint-Michielsgestel). The members of each pair knew each other well and frequently interacted with each other in daily life. The participants were asked to perform tasks like retelling narratives based on cartoons, comic stories and signed fable stories, as well as engaging in (semi-)spontaneous conversation and discussion of topics regarding deafness and sign language issues (Crasborn \& Zwitserlood, 2008b). The great majority of signers in the recordings have been educated at minimally secondary school level, with Dutch as the primary language of instruction and NGT playing a secondary role at best. NGT was for none of the signers a subject language in school, but had started to make its way into education for the younger signers as a language of instruction. In most cases, the language of instruction was spoken Dutch (for the older generations) and spoken Dutch alternated with signsupported speech for children educated from 1980 onwards (people younger than 30 in the corpus). The Corpus NGT is the best effort to date to record a representative sample of NGT use by the core of the deaf community, consisting of born-deaf or early-deafened signers, most of whom have not grown up in deaf families.

The typical duration of the recording sessions was four hours including explanation. This resulted in approximately 1.5 hours of usable data per signer pair, totalling 72 hours for the whole video collection. A deaf research assistant was present to guide the session. Each signer was recorded with two cameras: one HDV camera recording an upper body view and a DV camera recording a top view. The high definition upper body view recordings allowed for an MPEG-1 cut-out view with a close-up of the face. The sessions were cut into 2375 smaller videoclips so that each videoclip contained a particular topic (like retelling a Tweety \& Sylvester cartoon or a 
discussion about personal experiences). Annotation was done with the ELAN annotation software, developed at the Max Planck Institute for Psycholinguistics, The Language Archive in Nijmegen, The Netherlands (tla.mpi.nl/tools/tla-tools/elan/; see also Wittenburg, Brugman, Russel, Klassmann, \& Sloetjes, 2006). The annotation guidelines (Crasborn \& Zwitserlood, 2008a) describe how the initial annotation was organised. Lacking a practical orthography for sign language, signs are glossed using common Dutch words as a reference (so-called 'ID-glosses'; see Johnston, 2008). These are not translations, but rather pointers to a lexicon. The early annotators used the lexicon created by the Nederlands Gebarencentrum (Dutch Sign Centre; lexicon available on DVD and online: www.gebarencentrum.nl) as much as possible, but the glosses in the first release of the Corpus NGT contain many inconsistencies. Since then, glosses have been revised and they are much more consistent now; this is reflected in the recent second release of the corpus annotations.

Glosses refer to manual activity only, not to body or facial activity, regardless whether these express meaning such as negation. A notable exception to this occurs with homonyms. The signs for 'brother' and 'sister', for instance, are manually homonymous; since there is no Dutch word for 'siblings', they are glossed BROTHER and SISTER, depending on the mouthing that co-occurs with the sign. Glosses in the corpus are made in capital letters; throughout this thesis, small capitals will be used.

Between the moment of data selection for Chapter 2 and the moment of data selection for Chapter 5, a period of approximately four years, the corpus has grown from 55,000 to 137,000 gloss annotations for the combined left- and right-hand tiers. As of April 2014, the corpus contains more than 350,000 annotations, made for different levels of transcription and analysis, ranging from sentence translations (Ormel \& Crasborn, 2012) to degree of thumb extension (Ormel, Crasborn, \& De Meijer, 2013). Over the years, gloss annotations for manual signs have been added to the video recordings for different kinds of projects, with an emphasis on the Amsterdam and Groningen regions. Consequently, for the Amsterdam and Groningen regions, the annotations for mouth actions were also mainly done for signers from these regions in order to be able to study the coincidence of the two modalities.

\subsubsection{Annotation of mouth actions}

Corpus annotation is a process of constant decision making. Where does a sign, a mouth action, a sentence begin? Where does it end? What does it mean? How should 
it be transcribed? What annotations should be added? For mouth action transcription, one option is to describe them by their form, and use a classification of the amount of lip rounding, lip opening, and visibility of the tongue, for instance. These visible properties of articulations are accessible to deaf signers, and proposals for such 'viseme' categories have been suggested in the literature (see Massaro, 1998; Cappelletta \& Harte, 2012; Nonhebel, Crasborn, \& Van der Kooij, 2004). A transcription that is faithful to form is very useful for the notation of mouth gestures, and such system has been used in a previous project on mouth actions in the Corpus NGT (Van de Sande, 2009; Van de Sande \& Crasborn, 2009). For the notation of mouthings, although true to the form, a viseme notation would be very hard to read, however.

Another option for the transcription of mouth actions, one that is also not unproblematic but much more useful for investigating mouthings, aims at reconstructing words from a spoken language, using the phonological features that are visible. For vowels, lip rounding and jaw height (and thus tongue height) may be discriminated, but not front/back distinctions. For consonants, only the place of articulation may be clearly visible in the case of labials and dentolabials, but voicing and manner of articulation are very hard if not impossible to perceive. Thus, any attempt at reading speech involves a lot of interpretation; using a phonetic or orthographic transcription based on a spoken language would mean that a lot of inferences about what the signer might be saying have to be made on the basis of relatively little phonetic evidence. It may help in deciding on the transcription of the perceived mouthing to compare its meaning with the co-occurring sign, but this may be misleading in cases where the meaning of the mouthing differs from the meaning of the sign.

For efficiency reasons (see Johnston, 2010), an orthographic representation of the spoken language was used to transcribe mouthings for this thesis. After all, filling in details of spoken language articulations that cannot be perceived visually is not all that unnatural: deaf speechreaders do it all the time, and they are highly proficient in it (Woll, 2012). There is some evidence that deaf people are better at visual speechreading than hearing people (Auer \& Bernstein, 2007, Bernstein, Auer, \& Tucker, 2001). Moreover, listeners to natural speech do the same for missing auditory cues (e.g. Ernestus, 2000; Ernestus, Baayen, \& Schreuder, 2002; Mitterer \& McQueen, 2009). 
In deciding on the start and end points of mouth movements, the guidelines for annotating manual signs in the Corpus NGT (Crasborn \& Zwitserlood, 2008a) were adapted for the mouth:

- A mouth action begins on the video frame where the mouth starts moving from the previous movement or from the neutral state towards the target articulation(s).

- A mouth action ends when the mouth starts moving from the final articulatory position towards the next movement or towards the neutral position. However, if the final articulation is the same as a neutral state (such as $/ \mathrm{m} /$ followed by relaxed lips), a mouth action ends on the frame the final articulation is reached.

With the annotation of mouth actions, the first decision to be made is whether the mouth action in question is a mouth gesture or a mouthing. Since the research focus for this thesis is on mouthings and not on mouth gestures, the latter were only labelled according to their type and not further investigated. The different studies on mouthings each required their own set of annotation tiers; these will be described in more detail in the relevant chapters.

\subsection{Research questions and outline of this thesis}

The general aim of this thesis is to gain more insight in how Dutch spoken language intertwines with NGT in the form of mouthings. All data that we will report come from the Corpus NGT, meaning that we will be using elicited (semi)spontaneous, natural language, and we will be analysing an amount of data unprecedented in mouth action research. The annotations we created for our studies have been published in the second release of the Corpus NGT annotations, in the second half of 2014.

The overarching question about mouthings concerns their linguistic status: are they a form of code-mixing where signers may freely and creatively combine signs and mouthings, or have they become an inherent part of the sign language lexicon as component of the fixed phonological specification of a sign? We approached this overarching question from different angles or research questions, which will be elaborated on in the sections below: 
1. Do mouthings vary in relation to the signs they co-occur with? How frequent are temporal reductions in mouthings, and in what form do they occur? (Section 1.4.1, Chapter 2).

2. To what extent do we find spreading of mouthings over adjacent signs? Is there a preference for spreading over prosodically light elements like pointing signs? (Section 1.4.2, Chapter 3).

3. How prominent are mouthings in terms of frequency? Are there frequency differences when comparing age, gender, region, education, or having deaf or hearing parents? Could it be that some groups more often combine mouthings with pointing signs than with content signs? (Section 1.4.3, Chapter 4).

4. To what extent can NGT and spoken Dutch be combined to convey complex messages? Which word classes play a role in this bimodal code-mixing? (Section 1.4.4, Chapter 5).

Chapter 6 concludes with a discussion of the findings, where the relevance and implications of this study will be discussed in relation to possible directions for further research.

\subsubsection{Variation in mouth actions with manual signs (Chapter 2)}

Our first explorative study of the Corpus NGT was done from the perspective of the sign. We wanted to know how much variation there is in the use of Dutch lexical items as mouthings as they co-occur with manual signs. Finding no variation may suggest a fixed combination of sign and mouthing, and thus a possible lexical specification of mouthings in the sign language lexicon. Finding a lot of variation, on the other hand, may suggest that signers creatively and individually combine mouthings and signs to arrive at co-incidental practices of code-mixing to get meaning across. We took a sample of the 20 most frequently occurring signs in the corpus, leaving out indexical (pointing) signs and palm-up gestures, and investigated what the co-occurring mouth action was: a standard mouthing, a mouthing variant, a mouthing that originated in an adjacent sign, a mouth gesture, or no mouth action at all.

\subsubsection{The spreading of mouthings (Chapter 3)}

A number of tokens from the study on variation in chapter 2, recurrent for each of the 20 sign types, were cases where a sign co-occurred with a mouthing that was not "its own" - a mouthing that originated from an adjacent sign and spread over the sign in question. Sandler (1999; Nespor \& Sandler, 1999) showed for Israeli Sign Language 
that the articulation of mouthings can be delayed or extended with respect to the manual sign, so that two or more signs are realized during one mouthing. Sandler proposed that this may mark the prosodic domains 'prosodic word' and 'phonological phrase', suggesting that mouthings interact with prosody. Crasborn, Van der Kooij, Waters, Woll, and Mesch (2008) showed that similar processes take place in NGT and in British and Swedish Sign Language, and that there may be variation in the scope and direction of spreading. Their study, however, was based on a limited amount of data from a very limited number of people. We replicated the Crasborn, Van der Kooij et al. (2008) study, using much more data and many more participants, in a more natural language setting.

\subsubsection{The prominence of spoken language elements (Chapter 4)}

Given the changes in deaf education in recent decades as described in section 1.1.4, we were interested to see whether the change from oralism towards bimodal bilingual education is reflected in the use of mouthings by different age groups. Hoyer (2004) has suggested that mouthings are more frequently used by elderly signers in Finnish Sign Language; Mohr $(2011,2012)$ found both gender differences and age differences in a study on mouthings in Irish Sign Language. Previous studies on ASL (Nadolske \& Rosenstock, 2007) and NGT (Van de Sande, 2009) found register effects, with more mouthings being used in an interactive register rather than a narrative register. Still, when browsing the Corpus NGT, one gets the strong impression that there is a lot of variation in style of articulation between individual signers, both for manual and nonmanual features. We investigated various sociolinguistic variables, where we looked at the number of mouthings as compared to mouth actions, both for lexical signs and for pointing signs. Moreover, given that the number of mouth annotations in the Corpus NGT had more than doubled since the start of this project, it was also a good time to investigate how often mouthings actually occur in natural sign language use.

\subsubsection{Bimodal code-mixing (Chapter 5)}

Mouthings are usually analysed as having a one-on-one relationship with manual signs, both semantically and temporally. We found exceptions to semantical congruence between hands and mouth in the occurrence of non-standard mouthings in Chapter 1, and exceptions to temporal alignment of hands and mouth in the spreading of mouthings over adjacent signs in Chapter 2. In this final study, we further explored semantic and temporal incongruence. We looked in greater detail at 
mouthings that do not show a semantic overlap with the manual sign, identifying them as specifying mouthings (the non-standard mouthings we will encounter in Chapter 1). Further, we looked at mouthings that are added to the signing stream and do not accompany a corresponding sign. We identified these mouthings as solo mouthings and added mouthings, and make a sentence-level analysis of their occurrences. Terpstra and Schermer (2006), in an analysis of speech-supported Dutch, argued that there is a continuum with Dutch as its matrix language, blending varying levels of signing with it. We explored the hypothesis that a mirrored continuum exists for NGT, where NGT is the matrix language and spoken Dutch is blended in in a variety of ways. 


\section{Chapter 2: Variation in mouth actions with manual signs}

Slightly adapted from: Bank, R., Crasborn, O., \& Van Hout, R. (2011). Variation in mouth actions with manual signs in Sign Language of the Netherlands (NGT). Sign Language \& Linguistics, 14(2), 248-270. doi: 10.1075/sll.14.2.02ban

\subsection{Introduction}

\subsubsection{Background}

ign languages use the visuo-spatial modality. They are perceived by the eyes and articulated predominantly by the hands. However, much linguistic information is conveyed through non-manual articulation, such as eye gaze, body lean, and mouth actions, the subject of this paper. Two classes of mouth actions can be distinguished: mouth gestures and mouthings (Crasborn, Van der Kooij, Waters, Woll, \& Mesch, 2008), but see the various contributions in Boyes Braem and Sutton-Spence (2001) for discussions on terminology). Mouth gestures are defined as features inherent in the signed language that may or may not have an independent meaning and that may or may not be lexically bound to a manual sign (Crasborn, Van der Kooij et al., 2008: 50). Mouthings, on the other hand, originate in the 
surrounding spoken language and are lexically bound to the manual part of the signs. Although usually mainly associated with European sign languages, Nadolske and Rosenstock (2007) have shown that mouthings play an important role in American Sign Language (ASL) as well. Mouthings are silently mouthed instances of (parts of) spoken words, and are assumed to have the same meaning as their voiced spoken language counterparts. In this paper, we analyse mouthings as they co-occur with frequent signs in a corpus of Sign Language of the Netherlands (Nederlandse Gebarentaal, NGT). These mouthings are expected to be borrowed, originally, from the surrounding spoken language (for studies on various sign languages, see Ajello, Mazzoni, \& Nicolai, 2001; Bergman \& Wallin, 2001; Boyes Braem, 2001; Rainò, 2001; Vogt-Svendsen, 2001; Woll, 2001).

Borrowings enter a language as instances of code-mixing. Muysken (2000) defines code-mixing as a mixture of three different types of underlying processes:

insertion of material (lexical items or entire constituents) from one language into a structure from the other language

alternation between structures from languages

congruent lexicalization of material from different lexical inventories into a shared grammatical structure (Muysken, 2000: 3)

Generally, code-mixing is understood as occurring between two spoken languages. Muysken (2000) prefers 'code-mixing' as a neutral label to avoid complex discussions on concepts like code-switching and (nonce-)borrowing. Importantly, code-mixing refers to processes of sequential mixing. For the parallel processes of mixing signed language with spoken language, the term 'code-blending' has been proposed (Emmorey, Borinstein, \& Thompson, 2005). This term refers to forms of code-mixing where spoken words co-occur with signs. Emmorey et al. (2005: 666) define codeblends as "ASL signs [...] produced simultaneously with English words". We will use the term code-blending here as well to include co-occurrences of non-voiced words (i.e. mouthings) and manual signs, as far as these will turn out to be forms of codemixing. While some signers in the Corpus NGT make voiced sounds with their mouthings, we will consider voicing irrelevant here, because of the deafness of the interlocutors in the corpus.

Code-blending occurs on the continuum between fully signed and fully spoken utterances, and includes processes described by Muysken as insertion and alternation. In a longitudinal study on code-mixing between deaf mothers and their deaf or 
hearing children, Van den Bogaerde and Baker (2005) found that the "type of codemixing process that primarily occurred is congruent lexicalization with just some lexical insertion" (2005: 172). As will be described below, we will be concerned with mouth actions as they co-occur with single signs. We will therefore not be concerned with shared grammatical structures, and hence not with congruent lexicalization but with lexical insertion, a word-level process. In spoken languages, however, lexical insertion is a process where a lexical item of the matrix language is replaced by a lexical item of the second language, which in turn is inserted into the structure of the matrix language. While this may be true and even necessary for spoken languages, in bimodal code-mixing the articulators of the signed language are free to continue articulating whenever a spoken language element is inserted. It could be argued that this process is not really lexical insertion but rather lexical addition: addition of material (lexical items or entire constituents) from a language in one modality to material of a language in another modality. Since this is a word-level (or sign-level) process, potential differences in grammatical structures do not play a role here.

It has often been reported that mouthings mainly occur on nouns and nonmodified verbs (Boyes Braem \& Sutton-Spence, 2001: 4). Vogt-Svendsen (2001), for instance, reports this for Norwegian Sign Language. Schermer (1990) observes the same for NGT, but only in cases where mouthings are (temporally) reduced or serve to disambiguate, complement, or specify the manual part of the sign (that is, when the meaning of the mouthing is different from the manual sign, usually more specific). Non-reduced and redundant mouthings turn out to occur equally often with other grammatical categories as with nouns and verbs. We will make a distinction between word classes, including the distinction between content and function signs, as will be described below.

Temporal reduction is frequent in spoken language (cf. Cutler, 1998). Ernestus (2000) lists the numerous possibilities for reduction in casual spoken Dutch, ranging from absent consonants that have no visual cues (for instance, $[\mathrm{x}]$ or $[\mathrm{k}]$ ) to deletion of multiple segments of a word, for example deletion of unstressed syllables. Since casual spoken Dutch is an important source for mouthings in NGT, it is reasonable to assume that mouthings will show similar patterns of temporal reduction as in spoken Dutch, in particular for segment deletions. Alternatively, as reduction in speech is partly driven by acoustic output and auditory perception, the type of reduction processes in mouthings might also be very dissimilar, and based primarily on visual demands. 


\section{4 | Chapter 2: Variation in mouth actions with manual signs}

What can be said about the linguistic status of mouthings? Ebbinghaus and Heßmann (2001) argue that mouthings (as well as mouth gestures, for that matter) should not be regarded as parts of lexical signs, but rather as independent meaningful units that, in an interplay with manual signs, add to the overall meaning of the signed utterance. Vinson, Thompson, Skinner, Fox, and Vigliocco (2010) showed through experiments that the two modalities are represented and accessed largely independently from each other. On the other hand, Boyes Braem (2001: 128) suggests that "firmly established mouthings should be considered part of the sign language lexicon". There is a continuum between these two extreme positions. On one end of the continuum, mouthings can be seen as the outcome of online code-blending, where the user can freely choose between the various options that both (signed and spoken) languages offer. On the other end of the continuum, mouthings can be seen as fully lexicalized in the lexicon of the sign language, thus constituting an inherent part of the linguistic structure of the sign language. This would make mouthings in principle obligatory co-articulations for the user, although this may vary between signs.

\subsubsection{Research questions}

The current paper aims to investigate these two competing interpretations. When are mouthings "firmly established"? We studied the co-occurrence and variation of mouthings on the basis of a set of frequently occurring signs in the Corpus NGT (Crasborn \& Zwitserlood, 2008b; Crasborn, Zwitserlood, \& Ros, 2008). We will be looking for two kinds of variation in mouthings: variation in meaning and variation in timing. The first kind of variation can be characterized as lexico-semantic (i.e. mouthings that differ in meaning from the manual part of the signs); the second kind of variation has to do with temporal reduction (i.e. mouthings that only show a part of the corresponding spoken words, usually the first syllable(s)). Example (2.1) shows a case of lexico-semantic variation: the expected mouthing would have been gebaar ('to sign'). Example (2.2) shows temporal reduction of the Dutch word accepteren ('to accept') with additional regressive spreading of the reduced mouthing over the 
preceding pronoun. Please note that the extended lines following the mouthings in both examples only serve to (roughly) mark alignment with the manual sign. ${ }^{1}$

(2.1)

$\begin{array}{ll}\text { Manual } & \text { SIGN } \\ \text { Mouth } & \frac{\text { tolk }}{\text { 'interpreter' }} \\ \text { Meaning } & \text { Sign Language interpreter }\end{array}$

(2.2) Manual PT-1 ACCEPT

Mouth aksp

Meaning I accept

The present study seeks to answer the following questions: (1) How frequent are mouthings for NGT signs that have been observed with mouthings? (2) Within the class of mouthings, is there variation in the selection of spoken Dutch lexical items? (3) How frequent are temporal reductions in mouthings, and in what form do they occur? Answering these questions by investigating the most frequent signs in the Corpus NGT will shed light on the lexical status of mouthings. A high consistency in specific mouthing and manual sign pairs would suggest lexical specification of mouthings as a fixed component of the manual sign, originating as a borrowing from the spoken language. Alternatively, variability in the combination of mouthing and manual sign would point towards code-blending by the user, with mouthings not being an inherent part of the sign language lexicon. In these cases, the semantic context determines the choice of the spoken word. The possibility should also be considered that there can be multiple lexical specifications, in that some signs will have more than one lexicalized mouthing. This could be the case in, for example, minimal pairs like BROTHER and SISTER, in which the manual part is the same for the two signs but the mouthings are different, thus disambiguating the manual sign.

\footnotetext{
${ }^{1}$ The videos containing these examples are available in the online version of the Corpus NGT. Example (2.1) can be found at the following location:

http://corpus1.mpi.nl/qfs1/media-archive/NGT/Public/Media/CNGT0005.mpg

(at time point 05.41:160, signer on the right).

Example (2.2) can be found at::

http://corpus1.mpi.nl/qfs1/media-archive/NGT/Public/Media/CNGT0059.mpg

(at time point $01.03: 120$, signer on the left).
} 


\subsection{Methodology and data description}

The data are gathered from the Corpus NGT (Crasborn, Zwitserlood, \& Ros, 2008). The corpus was constructed between 2006 and 2008 and consists of 72 hours of recorded (semi-)spontaneous signing by 92 deaf signers from five different regions in the Netherlands (Amsterdam, Groningen, Voorburg, Rotterdam, and SintMichielsgestel). Some signers grew up in more than one region and thus were classified as coming from mixed regions. For the recordings, different tasks were used, such as retelling a fable story or discussions about 'deaf issues' like cochlear implants or sign language, in order to elicit different registers. At the moment of data selection for the current study, about $15 \%$ of the corpus was annotated in glosses for left and right hands, covering data from 68 signers. This yielded over 55,000 gloss tokens for more than 5,000 different types (of manual signs).

The choice of subjects was based on the annotation work already done by others (e.g. Van de Sande, 2009; Van de Sande \& Crasborn, 2009). This work provided a large data set already annotated with glosses for manual signs. These glosses are comparable with Auslan Corpus ID-glosses (e.g. Johnston, 2008) in that they should not be considered a translation of the sign but rather a unique identifier. However, contrary to the Auslan Corpus glosses, the Corpus NGT glosses are not yet based on an existing lexicon. ${ }^{2}$

The majority of this data set is taken from the recordings of signers from Amsterdam $(n=19)$ and Groningen $(n=31)$. To avoid possible peculiarities from the small numbers of signers from the other three regions, only this set of 50 signers was used in the current study. Table 2.1 provides the breakdown into age groups per area and gender.

\footnotetext{
${ }^{2}$ Currently, the glosses in the corpus are being revised in order to reflect a new lexicon that is created especially for the Corpus NGT. The selection of data presented here, although compiled before this revision started, has already benefitted from these revisions, because of the standardization of glosses used for the lexicon.
} 
Table 2.1. Participants in the current study, per age group, area and gender $(M=$ male, $\mathrm{F}=$ female).

\begin{tabular}{lccc}
\hline \hline Age group & Amsterdam $(\mathrm{M} / \mathrm{F})$ & Groningen $(\mathrm{M} / \mathrm{F})$ \\
\hline $15<25$ & $3(1 / 2)$ & 7 & $(3 / 4)$ \\
$25<35$ & $2(0 / 2)$ & 7 & $(4 / 3)$ \\
$35<45$ & $4(3 / 1)$ & 8 & $(2 / 6)$ \\
$45<55$ & $1(0 / 1)$ & 0 & $(0 / 0)$ \\
$55<65$ & $3(0 / 3)$ & 5 & $(3 / 2)$ \\
$65<75$ & $4(2 / 2)$ & 3 & $(3 / 0)$ \\
$75<85$ & $2(0 / 2)$ & 1 & $(0 / 1)$ \\
total: & $19(6 / 13)$ & $31(15 / 16)$ \\
\hline \hline
\end{tabular}

Within this subset of recordings from 50 signers, the most frequently occurring signs were identified. We did not differentiate between storytelling and discussion settings: while the proportion of lexical signs may be smaller in fables than in discussions (Van de Sande \& Crasborn, 2009), we have no reason to assume that mouthings for lexical signs will be different. Table 2.2 lists the glosses of the 30 most frequent signs of the Amsterdam and Groningen signers in the corpus, regardless of register. ${ }^{3}$

\footnotetext{
${ }^{3}$ We use the gloss PT- 1 to annotate pointing signs made with an index finger that are $1^{\text {st }}$ person pronouns. PT is used for all other pointing signs made with an index finger, usually pronouns or demonstratives. PU stands for 'palms-up', a gesture made with the palms of the hands oriented upwards.
} 
Table 2.2. Glosses for the 30 most frequent signs in the annotated Corpus NGT by signers from the Amsterdam and Groningen areas (November 2009).

\begin{tabular}{llllll}
\hline \hline Gloss & No. of signs & Gloss & No. of signs & Gloss & No. of signs \\
\hline PT & 2677 & 1 & 195 & SAME & 110 \\
PT-1 & 1661 & ALSO & 194 & IN & 101 \\
PU & 1653 & SAY & 193 & THINK & 98 \\
DEAF & 522 & BUT & 163 & GO & 96 \\
SIGN & 313 & SCHOOL & 160 & REMAIN & 90 \\
NOW & 253 & NOT & 147 & NAME & 87 \\
CAN & 252 & SEE & 131 & CI & 86 \\
HEARING & 212 & HAVE & 125 & BORN & 86 \\
YES & 209 & PREVIOUS & 123 & UNDERSTAND & 86 \\
GOOD & 203 & SELF & 114 & HANDICAPPED & 84 \\
\hline \hline
\end{tabular}

The signs in Table 2.2 cannot easily be classified according to word classes. The most prominent example is the sign SIGN: looking at the isolated manual sign, there is no way to distinguish between its verbal ('to sign') and nominal ('sign' or 'signs') use. The same is true for HEARING, which (again in isolation) can be an adjective ('hearing') or a verb ('to hear'), but the only way to decide is to perform a syntactic analysis including the surrounding context.

As mentioned earlier, mouthings are said to occur more frequently with content signs than with function signs (see, for example, Boyes Braem \& Sutton-Spence, 2001: 4). Therefore, variation is best investigated for the content words that are most frequent in the corpus. A few frequent function words (like ALSO, SELF) were also included in the selection to test this hypothesis. Twenty target signs were selected, listed in Table 2.3. 
Table 2.3. Target signs to be annotated for mouth actions in the Corpus NGT.

\begin{tabular}{llll}
\hline \hline ALSO & GOOD & PREVIOUS & SIGN \\
BORN & GROUP & SAY & TALK \\
CAN & HANDICAPPED & SCHOOL & THINK \\
CI & HEARING & SEE & UNDERSTAND \\
DEAF & NAME & SELF & YEAR \\
\hline \hline
\end{tabular}

The initial intent was to select 100 tokens per sign and to annotate those for mouth actions. This was accomplished for DEAF, CAN, and SIGN; however, for some of the selected signs, there were not enough tokens. The new target was set at twenty tokens to be annotated for mouth actions. For all selected tokens, the immediately surrounding signs were also annotated, to accommodate further research on the context of mouthing occurrences. This explains the variable number of tokens per sign that we report on. In order to represent the ratios of annotations per signer, the tokens were selected so that they were evenly spread over the annotated corpus (for instance, if 100 tokens had already been annotated for the hands, we would annotate every fifth occurrence for the mouth to get 20 mouth tokens).

For each token, an annotation was made for the mouth action accompanying the manual sign. This work was done by the first author, a native speaker of Dutch with moderate signing skills. Mouth actions that were judged to be (part of) a Dutch lexical item were annotated both for what was seen on the lips and for their spoken Dutch citation form (i.e. the infinitive for verbs, singular for nouns). Mouth actions that were judged to be mouth gestures were grouped according to their type (adverbial/adjectival, empty, whole face, and mouth for mouth; see Crasborn, Van der Kooij et al. 2008), but not further described in detail.

One of the problems in interpreting mouthings is that much of the articulated information is not visible to the interlocutor (or the researcher, for that matter). Specifically, although labial and dentolabial consonants (like /f/ and /p/, for instance) and some features of vowels (e.g. lip rounding, jaw height) may be clearly visible for the interlocutor, other consonants and the front/back distinctions in vowels are 
harder to distinguish, if at all. ${ }^{4}$ This poses a challenge for the annotation of mouthings. We made an educated best guess of the perceived actual word articulated by the signer, and included a second annotation on the same timeslot for the uninflected word, if applicable, or for the non-reduced form in case of temporal reduction. This strategy provides a relatively good connection between mouthing and intended meaning. However, as large parts of the articulated speech are not visible to the interlocutor, annotations are probably not $100 \%$ accurate concerning perceived intended speaker articulation. Nonetheless, they are relatively good in determining the intended meaning. Here, it is assumed that signers, when mouthing a spoken language item, articulate mouthings in the same way a hearing speaker would do with the vocalized spoken language counterpart of the mouthing.

Annotations were made using the ELAN annotation software (tla.mpi.nl/tools/tla-tools/elan/). In deciding on the start and end of mouth movements, the following guidelines were used (adapted from the guidelines for annotating manual signs in the Corpus NGT, in Crasborn \& Zwitserlood, 2008a):

- A mouth action begins on the video frame where the mouth starts moving from the previous movement or from the neutral state towards the target articulation(s).

- A mouth action ends when the mouth starts moving from the final articulatory position towards the next movement or towards the neutral position. However, if the final articulation is the same as a neutral state (such as $/ \mathrm{m} /$ followed by relaxed lips), a mouth action ends on the frame the final articulation is reached.

When one of the hands blocked the view of the mouth, or when the head was bent in such a way that the mouth was invisible, mouth actions were marked as 'not visible' on the mouth movement and type tiers. These instances were discarded for the current project.

${ }^{4}$ Actually, tongue height is the relevant feature for spoken language, but it is invisible to the interlocutor. Jaw height gives a means to estimate tongue height. 


\subsection{Results}

\subsubsection{Coding categories and quantitative results}

We used a coding scheme to qualify all mouth actions and mouthings. Five coding categories were required to distinguish the relevant mouth actions (or absence thereof, category 5):

1. Standard mouthing: forms of the spoken lexical items that occur most frequently with a manual sign (all reduced variants are grouped here as well). De facto, the standard mouthing is the one that has the same meaning as the manual sign.

2. Mouthing variant: forms of spoken lexical items that differ from the standard mouthings (as in example 2.1).

3. Overlap: instances where a manual sign is not accompanied by its own mouth action, but instead co-occurs with mouth actions of its neighbours. In example (2.3), the (standard) mouthing that co-occurs with LEARN completely overlaps the following sign SIGN. ${ }^{5}$ The next sign (CHILD) is the first of a new sentence.

$$
\begin{aligned}
& \text { (2.3) Manual: SIGN WANT LEARN SIGN // CHILD ... } \\
& \text { Mouth: <mouth gesture }>\text { wil leren kind ... } \\
& \text { 'want' 'learn' 'child' }
\end{aligned}
$$

4. Mouth gesture: mouth actions inherent to signed language, coded according to Crasborn, Van der Kooij et al. (2008), but not investigated further here.

5. No mouth action: mouth remains in neutral position.

${ }^{5}$ This example can be found at: http://corpus1.mpi.nl/qfs1/media-archive/NGT/Public/Media/CNGT0098.mpg (at time point 03.11:820, signer on the left). 


\section{2 | Chapter 2: Variation in mouth actions with manual signs}

We did not look at the exact alignment of mouthings with the manual part of the signs. For illustration, consider the example in Figure 2.1. In this example, the start and end of the mouthing jaar ('year') do not align with the manual sign YEAR (glossed as JAAR-B in Figure 2.1). ${ }^{6}$ However, it is clear from the sign/mouthing stream that jaar co-occurs with the manual sign JAAR.

\begin{tabular}{|c|c|c|c|c|c|c|c|}
\hline & $02: 20.500$ & $00: 02: 21.000$ & $00: 02$ : & 21.500 & $00: 02: 22.000$ & $00: 02: 22.500$ & 00:02 \\
\hline GlossL S1 [65] & & & & JAAR-B & & HUIS-A & VI \\
\hline GlossR S1 [198] & 3-B & KEER & \#PER & JAAR-B & NAAR & HUIS-A & \\
\hline Mouth S1 [25] & |'drie' & |'maal' & |'per' & & |'jaar' & |'naar'||'huis' & \\
\hline Mouth_TransI.S1 [7] & 'three & times & per & & year & house & \\
\hline
\end{tabular}

Figure 2.1. Example of partial alignment (JAAR with jaar 'year') and non-alignment (NAAR with naar 'to') of manual signs and mouthings.

There are a few cases in the sample where mouthings would not overlap at all with the manual sign. However, when it was clear that such an unlinked Dutch lexical item had the same meaning as the manual sign, the mouthing was counted as co-occurring with a sign, and not as 'overlap' or 'no mouth action'. In Figure 2.1, this is illustrated by the mouthing naar ('to'), which does not overlap with, but clearly 'belongs to' the manual TO (glossed as NAAR in the example).

The quantitative results are shown in Table 2.4 and Figure 2.2. Table 2.4 specifies the types of mouth action for the twenty most frequent signs in the Corpus NGT, with the number of selected tokens. The table shows variation in the frequencies of the tokens. The results in Table 2.4 are visualized in Figure 2.2.

${ }^{6}$ Unfortunately, this example is not available in the online version of the Corpus NGT for privacy protection reasons. 
Table 2.4. Percentages of tokens of signs (with number of tokens in parenthesis) where these signs are accompanied by standard mouthing, mouthing variant, overlap from adjacent signs, mouth gesture or no mouth action. Glosses are ordered by percentage of standard mouthings.

\begin{tabular}{|c|c|c|c|c|c|c|}
\hline \multicolumn{2}{|l|}{ GLOSS (token count) } & \multirow{2}{*}{$\begin{array}{l}\begin{array}{l}\% \\
\text { standard } \\
\text { mouthing } \\
\text { (tokens) }\end{array} \\
100(87)\end{array}$} & \multirow{2}{*}{$\begin{array}{l}\text { \% } \\
\text { mouth- } \\
\text { ing } \\
\text { variant } \\
\text { (tokens) } \\
0\end{array}$} & \multirow{2}{*}{$\begin{array}{l}\% \\
\text { over- } \\
\text { lap } \\
\text { (tokens) } \\
0\end{array}$} & \multirow{2}{*}{$\begin{array}{l}\text { \% } \\
\text { mouth } \\
\text { gesture } \\
\text { (tokens) }\end{array}$} & \multirow{2}{*}{$\begin{array}{l}\% \\
\text { no } \\
\text { mouth } \\
\text { action } \\
\text { (tokens) }\end{array}$} \\
\hline SCHOOL $(\mathrm{n}=87)$ & $\mathrm{N}$ & & & & & \\
\hline UNDERSTAND $(\mathrm{n}=19)$ & $\mathrm{V}$ & $100(19)$ & 0 & 0 & 0 & 0 \\
\hline YEAR $(n=28)$ & $\mathrm{N}$ & $96(27)$ & 0 & $4(1)$ & 0 & 0 \\
\hline $\operatorname{DEAF}(\mathrm{n}=104)$ & Adj & $95(99)$ & 0 & $1(1)$ & $2(2)$ & $2(2)$ \\
\hline ALSO $(n=22)$ & Adv & $95(21)$ & $5(1)$ & 0 & 0 & 0 \\
\hline HANDICAPPED $(\mathrm{n}=34)$ & Adj & $94(32)$ & 0 & $3(1)$ & $3(1)$ & 0 \\
\hline HEARING $(\mathrm{n}=70)$ & $\operatorname{Adj} / \mathrm{V}$ & $94(66)$ & $3(2)$ & 0 & $3(2)$ & 0 \\
\hline $\operatorname{NAME}(\mathrm{n}=15)$ & $\mathrm{N}$ & $93(14)$ & 0 & 0 & 0 & $7(1)$ \\
\hline CAN $(n=108)$ & $\mathrm{V}$ & $88(95)$ & $1(1)$ & $5(5)$ & $6(6)$ & $1(1)$ \\
\hline $\operatorname{SELF}(\mathrm{n}=44)$ & Dem & $86(38)$ & $7(3)$ & 0 & $2(1)$ & $5(2)$ \\
\hline PREVIOUS $(\mathrm{n}=29$ ) & Adj & $79(23)$ & 0 & $3(1)$ & $17 \quad(5)$ & 0 \\
\hline $\operatorname{SAY}(\mathrm{n}=31)$ & $\mathrm{V}$ & $77(24)$ & $3(1)$ & $3(1)$ & $13(4)$ & $3(1)$ \\
\hline BORN $(\mathrm{n}=29)$ & Adj & $68(19)$ & 0 & $7(2)$ & $18(5)$ & $7(2)$ \\
\hline THINK $(\mathrm{n}=43)$ & $\mathrm{V}$ & $60(25)$ & $7(3)$ & $2(1)$ & 21 & $10(4)$ \\
\hline $\operatorname{TALK}(\mathrm{n}=26)$ & $\mathrm{V}$ & $58(15)$ & 0 & 0 & $38(10)$ & $4(1)$ \\
\hline $\operatorname{GOOD}(\mathrm{n}=41)$ & Adj & $44(18)$ & $10(4)$ & $2(1)$ & $44(18)$ & 0 \\
\hline $\mathrm{CI}(\mathrm{n}=24)$ & $\mathrm{N}$ & $42(10)$ & $4(1)$ & $4(1)$ & $42(10)$ & $8(2)$ \\
\hline $\operatorname{SEE}(n=37)$ & $\mathrm{V}$ & $41(15)$ & $5(2)$ & $3(1)$ & $41(15)$ & $11(4)$ \\
\hline SIGN $(\mathrm{n}=136)$ & $\mathrm{V} / \mathrm{N}$ & $27(37)$ & $2(3)$ & $3(4)$ & $63(86)$ & $4(6)$ \\
\hline GROUP $(n=29)$ & $\mathrm{N}$ & 21 & $28(8)$ & $14(4)$ & $31 \quad(9)$ & $7(2)$ \\
\hline Mean percentage: & & 72.90 & 3.75 & 2.70 & 17.20 & 3.45 \\
\hline
\end{tabular}




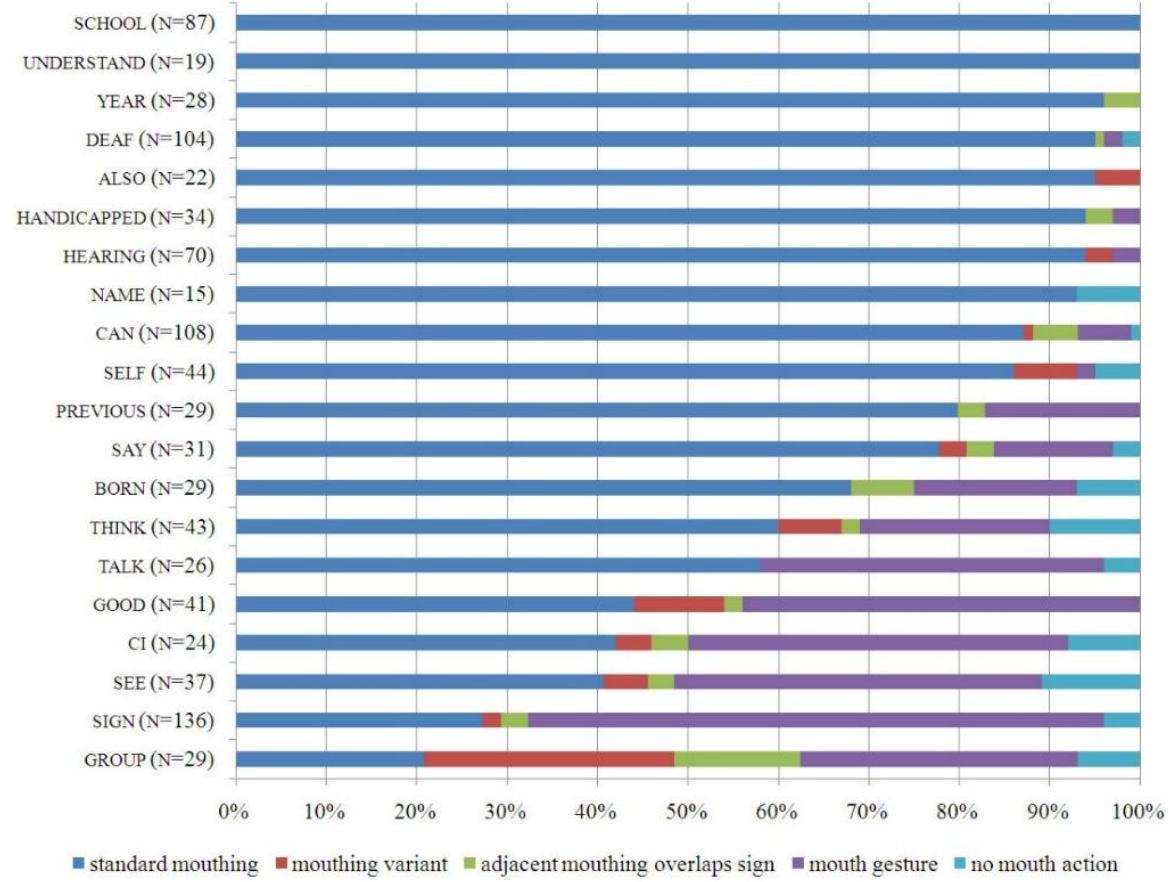

Figure 2.2. Distribution of the different types of mouth action for 20 frequent signs in the corpus NGT.

It is evident from Table 2.4 and Figure 2.2 that there is a strong consistency in the form of mouthings for many signs in the sample: $60 \%$ of the signs occur with the same mouthing of a (part of) a Dutch lexical item in over $75 \%$ of their tokens. The mean percentage of all standard mouthings is $72.9 \%$; five signs have percentages lower than $50 \%$. High scores are found for verbs, nouns, adverbs/adjectives, and the demonstrative SELF. There seems to be no word class specific pattern. The number of standard mouthings decreases with an increasing number of mouth gestures. The correlation is $-.652(p=.000, \mathrm{df}=18)$, indicating that these two categories are the most substantial ones and that they behave in a complementary way.

\subsubsection{Mouthing variants}

There are eleven signs that are accompanied by a spoken lexical item that is different from the standard mouthing (see column 'mouthing variant' in Table 2.4). The category 'no mouth action' is infrequent for all signs. 
Table 2.5. Signs having a mouthing variant, with translation, number of tokens and the percentage of the total number of mouthings (standard plus variants).

\begin{tabular}{|c|c|c|c|c|c|}
\hline \multirow{2}{*}{$\begin{array}{l}\text { Gloss } \\
\\
\text { CAN } \\
\end{array}$} & \multirow{2}{*}{$\begin{array}{l}\text { Mouthing variant } \\
\mathrm{mag} \\
\end{array}$} & \multirow{2}{*}{$\begin{array}{l}\text { Translation } \\
\text { 'may' }\end{array}$} & \multirow{2}{*}{$\begin{array}{l}\begin{array}{l}\text { No. of } \\
\text { tokens }\end{array} \\
(1) \\
\end{array}$} & \multicolumn{2}{|c|}{$\begin{array}{l}\text { Percentage of } \\
\text { total no. of } \\
\text { mouthings }\end{array}$} \\
\hline & & & & 1 & 1 \\
\hline $\mathrm{CI}$ & $o p$ & 'on' & $(1)$ & 8 & 8 \\
\hline \multirow[t]{3}{*}{ GOOD } & goeie & 'good one' & $(2)$ & 9 & \multirow{3}{*}{19} \\
\hline & belangrijk & 'important' & $(1)$ & 5 & \\
\hline & pema ('prima') & 'great' & $(1)$ & 5 & \\
\hline \multirow[t]{2}{*}{ GROUP } & gezin & 'family' & $(1)$ & 7 & \multirow{2}{*}{57} \\
\hline & klas & 'class' & $(7)$ & 50 & \\
\hline HEARING & slechtho ('slechthorend') & 'hard of hearing' & $(1)$ & 1 & 1 \\
\hline SELF & eigen & 'own' & (3) & 7 & 7 \\
\hline SAY & $\operatorname{vraaf}($ 'vraag af') & 'to wonder' & $(1)$ & 4 & 4 \\
\hline \multirow[t]{2}{*}{ SEE } & visueel & 'visual' & $(1)$ & 6 & \multirow{2}{*}{12} \\
\hline & kijk & 'to look' & $(1)$ & 6 & \\
\hline \multirow[t]{3}{*}{ SIGN } & tolk & 'interpreter' & $(1)$ & 3 & \multirow{3}{*}{9} \\
\hline & (?)hoeft niet & 'not necessary' & $(1)$ & 3 & \\
\hline & gaat & 'to go $\left(2^{\text {nd }} / 3^{\text {rd }}\right)$ ' & (1) & 3 & \\
\hline THINK & geloof & 'to believe' & (3) & 11 & 11 \\
\hline ALSO & vind & 'to find' & (1) & 5 & 5 \\
\hline
\end{tabular}

Table 2.5 lists the eleven signs with a mouthing variant, with percentages of the variants in relation to the total number of mouthings. For example, SEE occurs with two mouthings that differ from the standard zien, namely visueel ('visual') and kijk ('look'), each with one token (this makes up 12\% of the total number of mouthings occurring with this sign, and $5 \%$ of the total number of its tokens, see Table 2.4). As can be seen in Table 2.5, the number of tokens of mouthing variants is fairly small.

The list of mouthing variants in Table 2.5 shows that most variants are within the same semantic field as the standard mouthing. For example, GROUP has two variants that differ from the standard groep ('group'), namely gezin ('family') and klas ('class'). Both variants refer to a specific type of group. The signs CAN, GOOD, GROUP, HEARING, SELF, SAY, SEE, and THINK only have mouthing variants that are semantically 
related. This leaves us with three signs where the meaning of the mouthing variant is not directly related to the meaning of the standard mouthing: CI, HEARING, and SIGN. CI has one mouthing variant $o p$ ('on'), occurring once in the sample. It is used to indicate that a baby has a $\mathrm{CI}$ on its head, although the signer made the sign on her own head (which is the default location in non-locative use). For HEARING, the variant slechthorend ('hard of hearing') may be considered a specification of the manual part. Finally, for SIGN, two clear examples of mouthings unrelated to the standard are found: tolk ('interpreter'; see example 2.1) and hoeft niet ('not necessary'). These mouthings complement the sign, adding new information to the clause.

\subsubsection{Temporal reduction}

Tables 2.6 and 2.7 give information on the temporal reduction of the standard mouthings. We focus on the citation forms of the spoken Dutch mouthings that contain two or three syllables. For all two-syllable words in the sample, stress is on the first syllable in spoken Dutch; for all three-syllable words, stress is on the second syllable. There are three signs with a three-syllable citation form: begrijpen with manual UNDERSTAND, gebaren with SIGN, and geboren with BORN. ${ }^{7}$ For these signs, a clear pattern emerges. Table 2.6 lists the occurring tokens, their frequencies, and percentages.

${ }^{7}$ In the corresponding Dutch nouns (begrip, gebaar and geboorte), stress is also on the second syllable. 
Table 2.6. Temporal reduction for mouthings with three-syllable citation forms in spoken Dutch. Syllables stressed in spoken Dutch are underlined.

\begin{tabular}{|c|c|c|c|c|c|}
\hline & $\begin{array}{l}\text { No. of } \\
\text { standard } \\
\text { mouthings }\end{array}$ & $\begin{array}{l}\text { All three } \\
\text { syllables }\end{array}$ & $\begin{array}{l}\text { First two } \\
\text { syllables }\end{array}$ & $\begin{array}{l}\text { Second } \\
\text { syllable } \\
\text { only }\end{array}$ & $\begin{array}{l}\text { Last two } \\
\text { syllables }\end{array}$ \\
\hline UNDERSTAND & 19 & $\begin{array}{l}\text { begrijpen } \\
3(16 \%)\end{array}$ & $\begin{array}{l}\text { begrijp } \\
12(63 \%)\end{array}$ & $\frac{(g) \text { rijp }}{3(16 \%)}$ & $\begin{array}{l}\text { grijpen } \\
1(5 \%)\end{array}$ \\
\hline SIGN & 37 & $\begin{array}{l}\text { gebaren } \\
11(30 \%)\end{array}$ & $\frac{g(e) \underline{b a(a)(r)}}{24(65 \%)}$ & $\frac{\text { baar }}{2(5 \%)}$ & \\
\hline BORN & 19 & $\begin{array}{l}\text { geboren } \\
6(32 \%)\end{array}$ & $\frac{g e b o(o)(r)}{12(63 \%)}$ & $\frac{b o o}{1(5 \%)}$ & \\
\hline
\end{tabular}

In all three spoken Dutch lexical items, stress is on the second syllable. This second syllable is the only one that is consistently mouthed in our sample, the variation concerns the other syllables. It turns out to be fairly common for these mouthings to be fully pronounced: in 16 to 32 percent of the tokens of a sign, all syllables are mouthed. But by far the most common type of temporal reduction is pronouncing only the first two syllables, which occurs in over 60 percent of the cases. That means that the numbers of tokens where only the second syllable is articulated is small.

Table 2.7. Temporal reduction for mouthings with two-syllable citation forms in spoken Dutch. Syllables stressed in spoken Dutch are underlined.

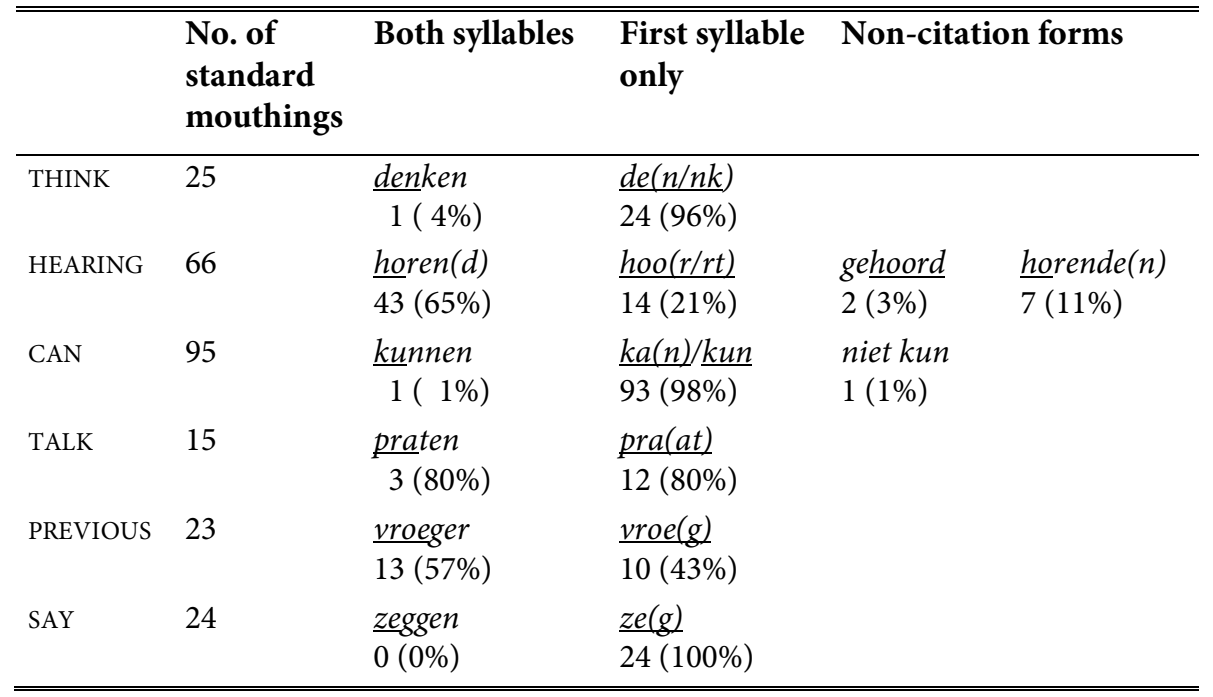


For two-syllable citation forms, the emphasis also lies on the stressed syllable. Table 2.7 lists the occurring tokens, their frequencies and percentages.

The results for the two-syllable citation forms are mixed. Some mouthings (those with THINK, CAN, and SAY) are almost exclusively reduced to a single syllable. For TALK, $80 \%$ is reduced, but PREVIOUS and the verb/adjective HEARING show a different pattern: there is a preference for the full two-syllable citation form. In more than half of the tokens of these signs, the mouthings are fully pronounced. Moreover, for HEARING, there are additional non-citation forms, one being the perfect participle gehoord ('heard'), the other an adjective or plural noun horende(n) ('hearing').

Finally, one-syllable citation forms are sometimes reduced to very short mouth movements: there is one occurrence in the sample where the standard mouthing that comes with SELF, zelf, is reduced to only $z f$ or $z(e) f$, a barely noticeable movement of the lower jaw. Another example is SEE, where the standard mouthing zien is reduced to only $z$. However, these occurrences are very infrequent and may be idiosyncratic. No examples of such extreme reductions are found in the mouthings accompanying other signs.

\subsection{Discussion}

\subsubsection{Frequency}

Our first research question concerned the frequency of mouthings with NGT signs that have been observed with mouthings. The data show that all signs in the twentysign sample often occur in combination with a mouthing, albeit in different proportions (from 29\% upwards). In the sample of the Corpus NGT that we have investigated, two signs even had $100 \%$ of their tokens occurring with mouthings, namely SCHOOL and UNDERSTAND. For both signs, the mouthings may have disambiguating functions: with $\mathrm{SCHOOL}$, the mouthing disambiguates the manual part from the otherwise homonymous WRITE and from one variant of NAME; for UNDERSTAND, the mouthing may disambiguate the sign from the manually identical STRANGE.

Further, for half of the signs in the sample, more than $85 \%$ of the tokens of those signs are accompanied by some sort of mouthing (including all lexical and temporal variation of mouthings). For the whole twenty-sign sample, the average 
percentage of mouthings co-occurring with a sign is $77 \%$ of its tokens $(72.90 \%$ standard mouthings plus 3.75\% variants). For mouth gestures, the average percentage of co-occurrence with a sign is $17 \%$ of its tokens; lower numbers of mouthings correlate with higher numbers of mouth gestures. Numbers for the 'no mouth action'category are rather small, varying between $0 \%$ and $10 \%$. For the current subset of signs, signers choose between using a mouthing and a mouth gesture. Considering the high percentages of mouthings per sign, the conclusion can be drawn that mouthings frequently accompany frequent signs. For some signs (SCHOOL and UNDERSTAND in this sample), mouthings might even turn out to be obligatory, if more data would confirm our findings on these items.

\subsubsection{Variation}

The second question concerned lexical and semantic variation in the Dutch lexical items used with mouthings. With the exception of GROUP (57\% mouthing variants, see Table 2.5), no more than $19 \%$ of the mouthed tokens of a sign differ from the mouthing that occurs most frequently, and which thus can be considered the de facto standard mouthing. There are four signs that have relatively many tokens where the mouthing differs from the standard: CI, GOOD, SEE, and THINK. All other signs have mouthing variants ranging from 0 to $10 \%$ of the standard. Within this small set of mouthings that differ from the standard, the great majority of mouthings are in the same semantic field as the standard mouthing. Hence, these variants can be seen as specifying manual signs that have a more general meaning. A special case in the sample may be GROUP, where the mouthing groep ('group') occurs slightly less often than one of its variants, klas ('class'). The reason that groep ('group') is still considered the standard mouthing is due to the fact that the concept 'group' has the most general meaning, which also motivates the gloss GROUP for the manual sign. It is likely that klas is a conventionalized mouthing to disambiguate the small difference in meaning. This is much like the case of BROTHER and SISTER: the signs are manually identical, but the mouthings provide a conventional semantic distinction, and both signs occur frequently in everyday language use. Accordingly, GROUP and CLASS have separate lemmata in the recently published Van Dale's Basic Dictionary of Sign Language of the Netherlands (Schermer, Koolhof, Muller, Geuze, \& Vink, 2009; see Zwitserlood, 2010, for a review). The findings in the corpus, reported here, support that decision, and argue for the same treatment in the corpus (i.e. introducing the gloss CLASs for the GROUP signs with the klas mouthing). 


\subsubsection{Temporal reduction}

Finally, the third question was how frequently temporal reduction of mouthings occurs, and in what form. Our findings indicate that the stressed part of the spoken language item is the most important one for the mouthing: all mouthings contain at least the stressed syllable. We observe frequent reduction of word-final schwa in full verb forms like praten ('talk') en kunnen ('can'). It is hard to generalize over the data because of the many differences in realizations, but the vast majority of tokens show word-final schwa deletion. We suggest two possible explanations for this: the fact that the last syllable contains a schwa, and inflection.

Schwa is a neutral vowel: its formant values lie in the middle of the vowel triangle, which is a consequence of the neutral position of the tongue (halfway front and back, and halfway closed and open; no lip rounding or widening of the mouth is present, the mouth is relaxed and slightly open). Therefore, in cases of word-final consonant deletion or whenever the word-final consonant articulation is invisible to the interlocutor, the annotation ends on the last frame of the second to last syllable. There is no way to decide whether the final syllable is intended to be pronounced or whether it is deleted. In this study, all two- and three-syllable mouthing citation forms end in a syllable with a schwa.

The other explanation of temporal reduction may be inflection. It could be the case that some verbs are inflected for their first, second, or third person singular form. In Dutch, these are formed by taking the stem of the verb (i.e. removing the infinitival suffix /-en/) for first person and by adding the suffix / $t$ / for second or third person. In, for example, zeg ('say') or zegt ('says'), the final consonant(s) would not be visible to the interlocutor (and neither would be the second syllable of longer words in casual speech). As another example, CAN is usually accompanied by the mouthing kan ('can', all persons singular; for $2^{\text {nd }}$ person this is the informal form), differing from plural (kunnen) or $2^{\text {nd }}$ person formal (kunt) by the absence of salient lip rounding. We did not systematically test this possibility by looking at the grammatical context of the sentence to see whether the inflected form would be appropriate. This would be an interesting next step in studying mouthings, as it could tell us whether only the Dutch lexicon is activated during sign language production or also the grammar of Dutch. At the very least, the stored inflected forms of Dutch verbs would be selected correctly, looking at morphosyntactic features in the sign lexicon.

It appears that the forms of temporal reduction that occur in mouthings have a lot in common with casual spoken language. The comparison is uneven because 
mouthings are only perceived visually - if there is any phonation, it can be considered irrelevant because of the deafness of the interlocutors in the corpus. In temporal reduction of mouthings, the part of the syllable structure that remains visible at all times is the stressed syllable, which is the most salient part of the spoken language word. However, reduction of the non-salient segments of the Dutch words is variable.

Apparently, there is no fixed form of a mouthing accompanying a sign, it can be reduced just like the corresponding spoken language forms. We consider this as support for the idea of code-blending signed and spoken language. This is in contrast with Schermer (1990), who does not consider temporally reduced mouthings as spoken language elements. She suggests that temporally reduced mouthings (reduced spoken components, in her terminology) behave like mouth gestures (oral components): "the spoken component is reduced in such a way that it is no longer identifiable by itself as a Dutch lexical item" (Schermer, 1990: 124). Although she does not provide a breakdown per sign, in her report, some signs end up in multiple classes (such as spoken or oral components) because of temporal reduction. While we found that signs indeed can be accompanied by one of multiple types of mouth action, we do not agree that temporal reductions should be classified as mouth gestures. Rather, as they alternate with full forms, it is likely that they correlate with other types of phonetic variation in the same context and that they can be recognized by the addressee as stemming from a Dutch word.

Although the aim of the present study was not to analyse the mouth gestures in any detail, it is clear that future work should focus on those as well. The substantial number of mouth gestures observed with some lexical items (e.g. up to $63 \%$ of all mouth actions for SIGN) calls for further research. The main question will be whether all mouth actions can be considered to have a modifying (adverbial, adjectival) function, or whether there are some meaningless or idiosyncratic mouth actions in these forms as well. We know that NGT has at least some items that do have a truly phonological mouth gesture which cannot be analysed as a morpheme, for example, the signs TO-BE-PRESENT (with a mouth gesture that is, in Dutch annotations, usually 
described as $\operatorname{sh}\left[\int\right]$ ) and OH-I-UNDERSTAND (with a mouth gesture usually described as van [ fan ] or fah [ fah ]). ${ }^{8}$

\subsection{Conclusion}

\subsubsection{General conclusion}

We have performed a corpus study in an annotated sign language video corpus to look for variation in occurrences of mouthings with manual signs. Our main finding is that, for the selection of highly frequent signs, there is little variation in the choice of the lexical items from the spoken language with a given manual sign. In that sense, mouthings for those signs can be said to be firmly established, as also suggested in Boyes Braem (2001: 128). However, we did find quite some variation in the type of mouth actions. With most signs, there is the possibility to use either a mouth gesture or a mouthing. While the distinction between mouthings and mouth gestures has been made by many researchers now, it is a striking new finding that some signs can be combined with either in NGT, presumably depending on the context. Verbs that can be accompanied by mouth gestures that have an adverbial function thus can also occur with a mouthing, it appears. While the use of oral adverbials has been well-described for languages like ASL (e.g. Liddell, 1980) and recently for BSL (Lewin \& Schembri, 2011), the alternate use of mouthings and mouth gestures accompanying nouns and adjectives is a new finding that demands further investigation. While some of the adjectives in our study might be analysed as adjectival predicates, at first sight, a verbal function does not appear to apply to the noun GROUP in NGT.

The reduction of mouthings and the other types of variation in mouth actions call for more research as well. While the present study clearly identified the reduction of mouthings to the stressed syllable in the Dutch word, showing that signers have access to the rhythmic structure of Dutch words even though their skills in Dutch are

\footnotetext{
${ }^{8}$ Some people have suggested that van of fah may be analyzed as a reduced mouthing, originating from vandaar ('Oh, I see'). However, we did not find any instances of the full mouthing vandaar in our corpus, and we believe that van or fah now should be analysed as a mouth gesture.
} 
highly variable, it is not clear how and to what extent further reductions (up to a mere onset consonant of the stressed syllable) take place. In addition, the use of inflected forms for verbs raises the question whether not only the Dutch lexicon is used by signers in blending Dutch words with manual signs, but also the morphosyntax of Dutch.

Even within the selection of highly frequent signs, substantial variation between signs was found in terms of the variation in mouth actions. Some signs showed much more frequent use of mouth gestures than others, for instance. This calls for a broader study of a larger repertoire of signs. Moreover, although we found little variation in the choice of Dutch lexical items for mouthings in frequent signs, the deaf assistants annotating the Corpus NGT regularly encounter unexpected mouthings, which for them creates confusion as to what the correct gloss should be. By extending our selection of signs to less frequent ones or to particular semantic domains, we might be able to corroborate this intuitive frequency of variation by empirical findings.

Earlier in this paper we suggested the term 'lexical addition' as a term for the word/sign-level processes of code-blending. While the term may be convenient for describing this level of code-blending occurrences, it may not reveal the true picture. We analysed signs with their mouth actions in isolation, not looking at their context. Consequently, any occurrences of processes of congruent lexicalization inevitably escaped our attention. Without looking at the grammatical structures, we simply cannot say whether any code-blending is the result of lexical addition or the result of congruent lexicalization.

\subsubsection{Concluding remarks on methodology}

A few remarks on the methodology of our study are in order. First, searching in an annotated corpus is not an easy task. For example, in the results section, the initial number of mouthing variants, based on a corpus search, was higher than it turned out to be after a closer look. CAN, for instance, appeared to have one occurrence of a mouthing annotated as meme [ $m \varepsilon m \varepsilon$ ], a nonsense word. The left adjacent sign had its own mouthing, the right adjacent sign was PT, a pointing sign - not a candidate for being the source of [ $m \varepsilon m \varepsilon$ ] either. Because of its bisyllabic structure it was initially classified as a mouthing, although it is semantically empty. A closer look at the sign, however, revealed that the mouthing was a false start for mee ('come along', 'with'), with the sign COME-ALONG occurring after the sequence CAN PT. Automatic 
processing of search results is excellent in most cases and gives reliable results for the most part, but peculiarities should be checked case by case.

Further, one might argue that the selection of target signs may have influenced the results. Tokens were selected evenly across the selected subset of the annotated corpus, so that signers of whom larger amounts of data had been annotated have more signs in the selection than signers for whom fewer annotations were available. This way of selecting was done in order to reflect the ratios of annotations per signer. However, things may have looked slightly different had the selection been genuinely random. Moreover, the annotated part of the corpus is continually changing and expanding. As mentioned before, glosses are currently revised in order to create a corpus-specific lexicon of ID-glosses. Although this has not influenced the selected tokens for this study, it may be that some tokens that were incorrectly glossed (for example, on the basis of a mouthing) are now corrected and have become part of the targeted signs for this study. We therefore checked whether the frequency list presented in Table 2.2 remains accurate, and this proved to be the case. Thus, we are confident that the ongoing revision of glosses will not alter the findings presented here.

Finally, in annotating the contexts of the target signs, sometimes a mouthing occurred with no corresponding sign. We did not systematically search the corpus for these instances, but it would be interesting to do so. Further, in ongoing work we are investigating the precise temporal alignment of signs and mouthings and mouth actions more generally, since we found during annotation for the present study that the start and end of mouthings do not necessarily align with the manual sign, in fact they might even not overlap at all. By investigating temporal alignment, we will gain insight into the freedom a signer has in combining spoken and signed language, and also into the prosodic structure of signed languages. This will also give us further clues as to the analysis of mouthings as part of the lexical entry of a sign vs. instances of code-blending.

To conclude, we would like to make a few remarks on the methodology of corpus studies like these. The use of a corpus for this kind of research has the great advantage that it allows us to observe what happens in spontaneous signing, rather than in signing in controlled settings. It thus provides us with quantitative data about what people actually do. Further, with the increasing functionality of annotation tools like ELAN, searching and annotating video corpora is becoming easier than ever before. However, it is important to carefully design the way a corpus will be annotated 
to allow for different search strategies and to properly document the annotation conventions to ensure consistency. Currently, there are not many sign language corpora in the world. The Corpus NGT is one of the bigger ones, and it is available online. This allows researchers throughout the world to conduct their own research on the corpus, but also to verify or falsify reported claims on NGT or sign language in general. 
46 | 


\section{Chapter 3: Alignment of two languages: The spreading of mouthings}

Slightly adapted from: Bank, R., Crasborn, O., \& Van Hout, R. (2013). Alignment of two languages: The spreading of mouthings in Sign Language of the Netherlands. International Journal of Bilingualism. Published online by Sage, May 3, 2013. doi:10.1177/1367006913484991

\subsection{Introduction}

@ ign languages are the principal means of communication in deaf communities. However, since they grow up in a hearing world, virtually all deaf signers are functionally bilingual: they have sufficient command of both their signed language and the surrounding spoken language for at least daily communication (cf. Ann, 2001; Lucas \& Valli, 1992). There are abundant examples of communities that mostly consist of bilingual speakers (Grosjean, 2010), including minority languages that only have bilingual speakers. Speakers of smaller languages are likely to also know a majority language (De Swaan, 2001). This is in fact what we see in deaf communities, where there is not a single situation in the world where sign language forms the dominant language; see Hiddinga and Crasborn (2011) for a discussion of the global situation of sign languages.

For the present generation of adult deaf signers in western countries, sign language is typically acquired informally from peers in school situations. Only a small 
minority of deaf people acquire their sign language from signing deaf parents, siblings, or deaf people in their extended family (Mitchell \& Karchmer, 2004). Spoken language, by contrast, has typically been taught formally in school programmes to all generations alive today. Deaf education throughout the $20^{\text {th }}$ century has seen a major focus on acquiring spoken language skills, which only in the last two decades has shown a slowly growing attention for sign language as a language of interaction between teachers and children, as a language of instruction, and as a subject language (Rietveld-Van Wingerden \& Tijsseling, 2010). Outside the western world, there is more variation in the impact of school settings on the acquisition of spoken language, with in certain cases no education for deaf children at all (Zeshan \& De Vos, 2012). The most pronounced examples of these situations are 'deaf villages' such as those in Bali (De Vos, 2012) where nearly all deaf people are monolingual signers, while a significant part of the hearing population is fluent in both the spoken and the sign language of the village. Martha's Vineyard (Groce, 1985) is a comparable case; see Nyst (2012) for an overview of such communities around the world.

There has recently been increasing attention for the resulting 'bimodal bilingualism', the combined knowledge of a spoken and a signed language, especially in the psycholinguistic literature (Emmorey, Borinstein, \& Thompson, 2005; Emmorey, Luk, Pyers, \& Bialystok, 2008; Hermans, Ormel, \& Knoors, 2010). While we tend to think first of all of deaf people as bimodal bilingual, there are substantial numbers of hearing people that acquire a signed and a spoken language from birth: both hearing children of deaf adults ('CODAs') and hearing siblings of deaf children in signing families with hearing parents can be considered fully bimodal bilingual, as they have full exposure to both the signed and the spoken language from an early age. Studies on language production of CODAs have shown there to be code-mixing phenomena of the same type as observed in spoken languages (Emmorey et al., 2005).

As a result of the bilingual nature of western deaf communities, spoken language items found their way into signers' communication. In spontaneous signing in Sign Language of the Netherlands (Nederlandse Gebarentaal, NGT), signs are usually accompanied by either mouthings or mouth gestures (e.g. Boyes Braem \& Sutton-Spence, 2001; Crasborn, Van der Kooij, Waters, Woll, \& Mesch, 2008; Schermer, 1990; Vogt-Svendsen, 2001). Mouthings are silently articulated words, or parts of words, originally stemming from the surrounding spoken language. They are, presumably, lexically bound to the manual part of the sign, since they are in general temporally aligned and share their meaning with the manual sign (Bank, Crasborn, \& 
Van Hout, 2011; Sutton-Spence, 2007). Mouth gestures are all other linguistically relevant mouth movements that occur with signs. Among the various functions of mouth gestures are adverbial or adjectival functions (such as puffed cheeks with the NGT sign HOUSE to indicate the big size of that house), they can enact the manual sign (such as a chewing movement with the sign CHEW), they can be part of a facial expression (such as an open mouth in a surprised look), or they can add to the phonological well-formedness of the sign (such as the pursed lips with the sign BE-PRESENT, cf. Vogt-Svendsen (2001) on Norwegian Sign Language, or Woll (2001) on BSL).

Schermer (1985) said that "the existence of a pure sign language, without the occurrence of any speech, among deaf adults, is more or less a theoretical construct" (1985: 288). The exact linguistic status of mouthings, however, is a source of ongoing debate. Various contributions to Boyes Braem and Sutton-Spence (2001), such as Ebbinghaus and Heßmann (2001), Hohenberger and Happ (2001) and Keller (2001), illustrate the different viewpoints, ranging from mouthings as parts of multichannel signs to mouthings as a totally independent channel. Indeed, Vinson, Thompson, Skinner, Fox, and Vigliocco (2010) found different error patterns for hand and mouth in a word-translation task and a picture-naming task. This suggests that, on a lexicosemantic level, the manual components and mouthings have separate representations for lexical signs. Thus, the combination of signs with mouthings can be characterised as code-blending (Emmorey et al., 2005), the simultaneous articulation of manual signs and spoken words.

Schermer (1985) predicted "that the role of speech in spontaneous [signing] will decrease in the future" (1985: 286) as a result of the decreasing influence of the oral tradition in the schools for the deaf. While we do not know whether Schermer's prediction has indeed come true, mouthings are still ubiquitous in NGT nowadays (Bank et al., 2011).

Importantly, mouth actions usually have roughly the same timing as the sign they occur with, and can thus be said to accompany manual signs. As previous research on highly frequent NGT signs has shown, these signs are not exclusively accompanied by either a mouth gesture or a mouthing (Bank et al., 2011). Signs vary in the way they co-occur with mouth actions, sometimes occurring with a mouth gesture, sometimes with a mouthing, and occasionally with no mouth action at all.

An interesting phenomenon is the loose character of the lexically boundedness' of mouthings. Bank et al. (2011) found that for most highly frequent 
signs there was at least one instance where a sign co-occurred with a mouthing related to the previous sign. In other words, the mouthing of the previous sign has spread over the next sign. In example (3.1), the signer says that she went to a doctor. The sign glossed as DOCTOR co-occurs with the onset of the mouthing dokter ('doctor'), and the following sign GO co-occurs with the rest of the mouthing dokter. There is no room for a mouth action to exclusively accompany the sign GO.

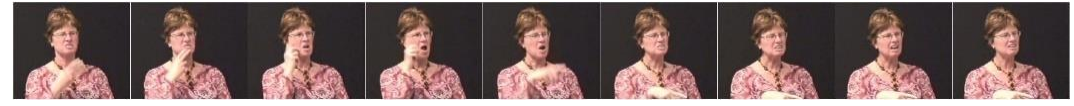

Manual: DOCTOR $\mathrm{GO}$

Mouth: d (k)t (r)

Meaning: 'I go/went to the doctor'

Spreading of mouthings has been described before by, among others, Boyes Braem (2001) for Swiss German Sign Language, Sutton-Spence (2007) for BSL, and Crasborn, Van der Kooij et al. (2008), who describe spreading in NGT, BSL and Swedish Sign Language (SSL). For NGT (as well as for BSL and SSL), they found that the majority of spreading mouth actions spread rightward (progressively) over only one additional sign. Occasionally, mouth actions would spread over more than one sign, or spread leftward (regressively), but such cases were rare. Spreading mostly occurred over a pointing sign (PT, plural: PTs), a prosodically light element that often does not have an autonomous movement unit (Crasborn, Van der Kooij et al., 2008: 62). This lack of inherent movement makes them easily absorbed by prosodically heavier signs (i.e. that have extensive specifications for location, movement and orientation), or in other words, they become clitics connected to the following or preceding sign. In her analysis of Israeli Sign Language (ISL), Sandler (1999) proposes the existence of the prosodic word as a constituent of ISL. The spreading of mouthings over adjacent signs is one of the pieces of evidence she uses to support the claim that a lexical sign and the following pronoun should be regarded as one prosodic constituent. Boyes Braem (2001) found that early learners use spreadings ('stretched mouthings' in her words, 2001: 106ff) more frequently than late learners, and distinguishes three prosodic functions of spreadings: to bind constituents of noun phrases, to bind verbs with subjects and to bind larger prosodic units. 
The data described in Crasborn, Van der Kooij et al. (2008) are based on a limited number of participants (two signers per language) signing stories to a camera. The current paper will build on their research. We will replicate the Crasborn, Van der Kooij et al. (2008) study, with a much larger corpus of 46 participants (age range: 17-82), in an everyday language use setting. Further, to make full use of the size of our corpus, we will take region, gender, and age into account in our analysis. Whether any of these sociolinguistic variables has any effect on the way NGT is used has not been thoroughly studied to date. We know that there are some regional lexical differences between the Groningen and Amsterdam dialects of NGT, but it will be interesting to investigate whether these signers also make different prosodic choices in their everyday signing. Further, with the wide range of signers age available to us in the corpus, we will be able to look at changes over age groups. If the prosodic structure of NGT has changed over the last few decades, we may expect this to turn up as differences between the age groups.

We will explore the following research questions. The first main question we will address is to what extent we find spreading of mouthings over neighbouring signs in conversational NGT. We will compare our results from a 46 signer corpus to the Crasborn, Van der Kooij et al. (2008) study that was done with two signers. Given the size of our corpus, we will address two additional questions related to the background characteristics of the learners and spreading: first, are there any region-, age- or gender-specific differences in spreading behaviour? And second, do early learners show the same spreading patterns as late(r) learners?

The second main question relates to the timing of spreading. Is there a preference for mouthings to spread over prosodically light elements like clitics, or does it occur over heavier elements as well? A preference for light elements would indicate that spreading is not merely an articulatory coincidence, where multi-syllable mouthings push themselves over the sign boundary to spread over the next sign, but a prosodic strategy to create single prosodic words composed of content words and functional elements (Sandler, 1999).

We will use the Corpus NGT (Crasborn \& Zwitserlood, 2008b; Crasborn, Zwitserlood, \& Ros, 2008) and analyse natural language use to find the answers to these questions. The next section will provide more information on this corpus and on our methodology. 


\subsection{Methodology}

\subsubsection{The Corpus NGT}

The Corpus NGT was recorded from 2006 to 2008, and contains data from all five regions in the Netherlands where deaf schools are located (Amsterdam, Groningen, Rotterdam, Voorburg and Sint-Michielsgestel). The corpus spans 72 hours of video of 92 signers, recorded in pairs. For the recording of the corpus, participants were selected on signing skill; all had followed secondary education and completed exams in spoken Dutch. All participants were prelingually deaf and started using NGT before the age of four. They were asked to perform tasks like retelling narratives based on cartoons, comic stories and signed fable stories, as well as engaging in (semi-)spontaneous conversation and discussion of topics regarding deafness and sign language issues (Crasborn \& Zwitserlood, 2008b).

Over the last few years, gloss annotations for manual signs have been added to the video recordings for different kinds of projects, with an emphasis on the Amsterdam and Groningen regions. Consequently, for the Amsterdam and Groningen regions, more manual data are available for mouth annotations to be based upon.

As for the annotation of mouth actions, a firm first step was set by Van de Sande and Crasborn (2009) with the full annotation of 16 clips, again mostly (but not exclusively) by Amsterdam and Groningen signers. Bank et al. (2011) focused on the 20 most frequent lexical signs of the Amsterdam/Groningen part of the corpus, such as SCHOOL, DEAF and HEARING. Annotations were made throughout this part of the corpus for those frequent signs, including a few adjacent signs. While this was considered the best method for data collection for that study, it resulted in annotations being scattered throughout the Amsterdam/Groningen part of the corpus. Other small projects on the mouth, such as a study on homonyms, added more isolated annotations. For the current paper, to fill up some of the gaps between any scattered annotations, additional annotations of all occurring mouth actions were made between isolated annotations, in order to create longer continuous stretches of mouth annotations.

In all, there are 219 clips containing mouth annotations for the Amsterdam/Groningen part of the corpus, some fully annotated, others containing shorter stretches of annotations or even only a few isolated ones. Initially, we made 
two sets of data for this paper. Sample 1 contained all available mouth annotations from all 219 clips, both isolated mouthings and continuously annotated stretches. The proportion of mouth annotations for highly frequent signs and for homonyms was relatively high in this sample, for reasons explained above. In order to prevent possible strange outcomes, we therefore created Sample 2, a subset of the Sample 1. Sample 2 contained only the data from the 42 fully annotated clips, and was therefore a more balanced sample. In using Sample 2, any irregularities that may have been caused by annotation choices made in previous projects would be cancelled out. Table 3.1 gives the number of mouthings for both samples.

Table 3.1. Frequencies of mouth actions and mouthings, both in Sample 1 and Sample 2.

\begin{tabular}{lll}
\hline \hline & Sample 1 frequency & Sample 2 frequency \\
\hline Mouth actions & 7979 & 4806 \\
Mouthings & 5929 & 3447 \\
\hline \hline
\end{tabular}

The differences between Sample 1 and Sample 2, however, turned out to be marginal. There were no significant differences in spreading between Sample 1 and 2, either for progressive or for regressive spreading. Calculations on scope and on spreading over PTs (see sections 3.3.2 and 3.3.3) on any of the sociolinguistic variables that we explored did not reveal any significant differences either. We therefore concluded that it is safe to only use Sample 1 for the current study and benefit from the larger numbers in that sample.

\subsubsection{Participants}

The signers from the Amsterdam and Groningen regions account for over half of the entire corpus (50 signers out of a total of 92). As explained above, the largest number of manual glosses is available for this group. Therefore, for the current study we concentrated on this subset of the Corpus NGT. With these 50 signers, we have 219 short video clips fully annotated for manual signs and (partly) annotated for mouth actions. It turned out that for four signers there were no annotated mouth actions, leaving us with 46 participants. 
When reporting on effects of language learning, usually the Age of Acquisition (AoA) is reported. In the present case, the AoA was self-reported by the signers by means of a questionnaire that the participants had to fill out when the video recordings of the Corpus NGT were made. Since fluent sign language acquisition is dependent on many factors, we felt that AoA, being self-reported, would not truly reflect a signer's sign language skills. Therefore, following Lucas, Bayley, and Valli (2001), we chose a division that was based upon the hearing status of the signer's parents. This serves as a good rough marker for AoA: if at least one of the parents was deaf, the participant would have sign language input from birth, from an experienced (not necessarily native) signer, and could hence be considered a true L1-learner. Thirteen out of 46 signers (28\%) had at least one deaf parent: 12 had two deaf parents, one had a deaf mother and a hearing father. In this paper, we will refer to these signers as native signers, vs. non-native signers who were born from two hearing parents. The signers we here consider as non-natives learned NGT from their peers and teachers at deaf schools, starting from four years and one month of age, on average.

Table 3.2 provides a breakdown into gender and three age ranges, for all participants vs. the subset of native signers.

Table 3.2. Breakdown into age groups and gender $(M=$ male, $F=$ female $)$ and number of participants with deaf parent(s), for all participants and for the subset of native signers.

\begin{tabular}{llc}
\hline \hline Age range & All participants (M/F) & Native signers (M/F) \\
\hline Younger (range 17-30, mean 23) & $13(7 / 6)$ & $3(1 / 2)$ \\
Middle (range 31-60, mean 43) & $21(7 / 14)$ & $7(5 / 2)$ \\
Older (range 61-82, mean 69) & $12(6 / 6)$ & $3(1 / 2)$ \\
All (range 17-82, mean 44) & $46(20 / 26)$ & $13(7 / 6)$ \\
\hline \hline
\end{tabular}

\subsubsection{Data}

Annotating the Corpus NGT is an ongoing process, for all aspects of the sign stream. We used the ELAN annotation software (Wittenburg, Brugman, Russel, Klassmann, \& Sloetjes, 2006) to annotate the mouth actions. Signs had been annotated following the 
guidelines in Crasborn and Zwitserlood (2008a), and the mouth annotation guidelines were adapted from those: signs and mouthings start on the first video frame where the hands or mouth start moving towards the target articulation, and they end on the frame before the movement towards the next target articulation (or neutral position) begins. In case the end of a mouthing had the same form as the start of the following mouth action (or neutral state, such as $/ \mathrm{m} /$ followed by closed lips), the mouthing would end on the frame where that following articulation (or neutral position) is started. Mouth annotations were made on four tiers for each signer, as explained in Table 3.3.

Table 3.3. Tier description for annotation of mouth actions in the Corpus NGT. Child tiers inherit temporal alignment from the main tier.

\begin{tabular}{ll}
\hline \hline Tier name & Description \\
$\begin{array}{l}\text { Mouth } \\
\text { (main tier) }\end{array}$ & $\begin{array}{l}\text { Main annotation tier for mouth actions. In case of mouthings, } \\
\text { the observed (interpreted) Dutch word that is mouthed is } \\
\text { inserted here, including any inflection and/or temporal } \\
\text { reduction. }\end{array}$ \\
$\begin{array}{l}\text { MouthType } \\
\text { (child of Mouth) }\end{array}$ & $\begin{array}{l}\text { Tier for the classification of the mouth action as mouthing (M) } \\
\text { or mouth gesture (A for adverbial/adjective, E for empty, 4 for } \\
\text { mouth-for-mouth, W for whole face; see Crasborn, }\end{array}$ \\
$\begin{array}{l}\text { Van der Kooij et al., 2008). } \\
\text { MouthLemma }\end{array}$ & $\begin{array}{l}\text { Tier for the dictionary version of a mouthing, i.e. the unreduced, } \\
\text { uninflected perceived intended meaning. }\end{array}$ \\
$\begin{array}{l}\text { MouthSpreading } \\
\text { (child of Mouth) }\end{array}$ & $\begin{array}{l}\text { Tier for the marking of spreading phenomena. Annotations list } \\
\text { the signs co-occurring with the mouthing and the direction of } \\
\text { spreading. }\end{array}$ \\
\hline \hline
\end{tabular}

A mouthing was understood to spread over a neighbouring sign if it continued to be present for at least $50 \%$ of that neighbouring sign's duration. The sign that is the source of the mouthing is the sign that has the closest semantic relation with that mouthing (usually a standard mouthing, see Bank et al., 2011). If the source sign was two-handed and both the non-dominant hand and the mouthing spread over a one-handed target sign, this would still be considered spreading of the mouth action, although the spreading of the non-dominant hand may phonetically be more prominent. When 
calculating the length of the source sign (see section 3.4.2) in such cases, the length of the annotation of the dominant hand was taken.

Annotations on the MouthSpreading tier were made in the form of [originating sign $>>$ next sign] or [previous sign $<<$ originating sign] to indicate progressive vs. regressive spreading, respectively. In cases where spreading would occur over more than one sign, the annotation would be extended with $[\ldots>>$ next sign] (or, in case of regressive spreading, [previous sign $<<\ldots]$ ).

After initial analysis of the data, we wanted to know whether there would be any differences between spreading occurring over PTs and spreading over nonpointing signs, because of the prosodic lightness of PTs (Crasborn, Van der Kooij, \& Ros, 2012; Sandler, 1999). This analysis showed that roughly a third of all PTs occurred with their own mouthing, not spreading from another sign. Since PTs in pronominal or demonstrative use only occasionally occur with their own mouthings (because their referents are usually present or become clear from the context; Sutton-Spence \& Day, 2001), we looked up all of these occurrences to see which mouthings were used with these PTs. We found that $28 \%$ of the tokens were false positives: PTs that seemed to occur with their own mouthing, but that were in fact overlapping with one or two frames of a mouth action from an adjacent sign. They were not annotated as 'spreading' because the overlaps were too short for that (i.e. less than $50 \%$ of the PT's duration).

Finally, one of the things we wanted to know was whether there is something in the mouthings that makes them spread. Are they longer than other mouthings? Or are the signs they occur with very short? Unfortunately, we do not have phonetic information of the signs (like path of movement, length of hold or sign-internal repetition). But we did transcribe mouthings as how they were pronounced, so we know how many syllables there are in a mouthing. We did this quick and dirty, and defined a syllable as any vowel or string of vowels that is preceded or followed by either a word boundary or a consonant. Section 3.4.1 will present the results for the length of mouthings. Section 3.4.2 will present the results on the length of the signs that are the source of the mouthings that spread over the next or previous sign. 


\subsection{Results on direction and scope}

At the time of data selection for the current study (i.e. after additional annotation work), there were 7,979 mouth actions in our sample, including 5,929 mouthings (74.3\%). Of all these mouthings, 810 (13.7\%) spread over one or more neighbouring signs. This percentage on spreading is based on the total number of spreadings in comparison to the total number of mouthings, not taking into account that some signers may contribute more to the average than others. The average of all individual percentages (mouthings compared to spreading) is $13.8 \%$, ranging from $0 \%$ to $22.7 \%$. We will report below on possible influences of region, age, gender or nativeness.

There are a few more annotations for female signers than for male signers, reflecting the larger number of females in the sample. The same applies to signers from the Groningen area compared to the Amsterdam area. Table 3.4 summarises the results, and also reports on the numbers of annotations per gender and nativeness. Further, frequencies for register (conversational or narrative) are given as well, although we did not investigate this further. Please note that the number of mouthings is a subset of the number of mouth actions, and that the number of spreadings is in turn a subset of the number of mouthings, as explained above.

Table 3.4. Number of annotations for mouth actions, mouthings and spreading: overall, per gender, per region, per age group, per having deaf parents or not, and per register.

\begin{tabular}{llll}
\hline \hline & Mouth actions & Mouthings & Spreadings \\
\hline Total number & 7979 & 5929 & 810 \\
Male / female & 3887 / 4092 & $2735 / 3194$ & $342 / 468$ \\
Amsterdam / Groningen & $2909 / 5070$ & $2182 / 3747$ & $331 / 479$ \\
Younger / middle / older & $1983 / 3125 / 2871$ & $1462 / 2214 / 2253$ & $194 / 324 / 292$ \\
Native / non-native & $2774 / 5205$ & $2063 / 3866$ & $289 / 521$ \\
Conversational / narrative & $6552 / 1427$ & $5223 / 706$ & $711 / 99$ \\
\hline \hline
\end{tabular}




\subsubsection{Direction of spreading}

Our sample contains 810 spreading mouthings. The majority of these mouthings (762, 94.1\%) spread rightwards (progressive), while only a small number spread leftwards (regressive) (42,5.2\%). The remaining six mouthings $(0.7 \%)$ spread from one of the middle ones of multiple signs. Although the 42 leftward spreadings take up only $5.2 \%$ of all spreadings, it is by no means an idiosyncratic process: 24 of the 46 signers do it sometimes. Table 3.5 summarises the spreading behaviour of all subjects.

Table 3.5. Frequencies and percentages for direction of spreading. Percentages on the right side are relative to spreading.

\begin{tabular}{lccc}
\hline \hline & Frequency & Percentage & \\
\hline Mouthings & 5929 & $100 \%$ & \\
Spreadings & 810 & $13.7 \%$ & $100 \%$ \\
Progressive spreading & 762 & & $94.1 \%$ \\
Regressive spreading & 42 & & $5.2 \%$ \\
Outward spreading & 6 & & $0.7 \%$ \\
\hline \hline
\end{tabular}

We performed a mixed model analysis on the sample data (random intercept model, with signers as a random effect) with a logit link function on the frequency of occurrence of spreadings and on the direction of these spreadings (SPSS 19, mixed models). We investigated whether age (in three age groups), region (Amsterdam, Groningen), gender and being a native signer (i.e. having at least one deaf parent, see section 3.2.2) had an effect on the frequency of occurrence, but none of these variables had a significant impact. Variation in frequency of regressive spreading seems to be bound primarily by individual differences. The number of regressive spreadings is moderately correlated to the number of spreadings at the signer level ( $r=.481, N=46, p=.001)$. This may indicate that other factors play a role, but it seems safer to conclude that regressive spreadings are not only rather infrequent, but that the variation in frequency is largely a signer-bound phenomenon. 


\subsubsection{Scope of spreading}

Most often, mouthings spread over only the immediately adjacent sign. This happened in 761 cases (94.0\% of all spreadings). But just as Crasborn, Van der Kooij et al. (2008) found, there is also spreading over more than one adjacent sign, although this does not happen frequently. There are 45 occurrences (5.6\%) of mouthings spreading over two signs, the pooled number of spreadings over three, four or five signs is four (0.5\%). Once again, spreading over more than one sign is not idiosyncratic: it is done by 23 signers. Table 3.6 summarises the results.

Table 3.6. Frequencies and percentages for spreading over multiple signs.

\begin{tabular}{lrc}
\hline \hline & Frequency & Percentage \\
\hline Mouthings & 5929 & \\
Spreadings & 810 & $100 \%$ \\
Spreading over 1 adjacent sign & 761 & $94.0 \%$ \\
Spreading over 2 adjacent signs & 45 & $5.6 \%$ \\
Spreading over 3/4/5 adjacent signs & 4 & $0.5 \%$ \\
\hline \hline
\end{tabular}

The number of multi-sign spreadings is highly correlated to the number of spreadings at the signer level $(r=.842, N=46, p=.000)$. More spreadings means more multi-sign spreadings, which applies to all signers. Given this strong relation, it is evident that no effects were found for age, region, gender or being a native signer on the occurrence of multiple-sign spreadings (mixed models analysis).

\subsubsection{Spreading over pointing signs}

The majority of spreading mouthings occur over PTs. These are typically pronouns, but can have various functions such as possessive, demonstrative or locative. Of all mouthings that spread, 472 (58.3\%) do so over a PT. Table 3.7 summarises the results. 
60 | Chapter 3: Alignment of two languages: The spreading of mouthings

Table 3.7. Frequencies and percentages for spreading over PTs. Percentages on the right side are relative to spreading over any PT.

\begin{tabular}{lrrc}
\hline \hline & Frequency & Percentage \\
\hline Mouthings & 5929 & & \\
Spreadings & 810 & $100 \%$ & \\
Spreading over any PT & 472 & $58.3 \%$ & $100 \%$ \\
Progressive spreading over 1 sign that is a PT & 403 & $85.4 \%$ \\
Regressive spreading over 1 sign that is a PT & 32 & $6.8 \%$ \\
Spreading over multiple signs, including any PTs & 37 & $7.8 \%$ \\
\hline \hline
\end{tabular}

The number of spreadings over any PT is very highly correlated to the number of spreadings at the signer level $(r=.932, N=46, p=.000)$. More spreadings means more spreadings over PTs, which applies to all signers. Given this strong relation, it is evident that no effects were found for age, region, gender or being a native signer on the occurrence of spreadings over PTs (mixed models analysis).

\subsubsection{Regressive spreading at the sign level}

Now that we have determined the basic spreading behaviour of mouthings, we will take a short closer look at regressive spreading. We examined the 42 left-spreading mouthings in our sample, to see whether there is anything they have in common. We found no distinctive pattern in regressive spreading. Source signs include verbs, nouns, adverbs and adjectives, as well as ordinals and interjections. Out of these 42 mouthings, 33 (79\%) spread over a PT (including THERE). Regressive spreading appears to occur mainly over PTs, but there are a few other cases that have to be accounted for. Lacking evidence for a more specific explanation, we keep open the possibility that leftward spreadings are merely errors in production planning. 


\subsection{Results on the relative length of spreadings}

Now that we have established that age, gender, region or having a deaf parent are not factors that can help us in understanding the spreading behaviour of mouthings, we want to test one specific hypothesis on the reason for spreading, namely that the length of the manual or mouthed units is somehow involved. First we will report on the length of the mouth part, both in milliseconds and in number of syllables (section 3.4.1), then we investigate the length of the sign part (section 3.4.2).

\subsubsection{Length of spreadings}

The average length of a mouthing that does not spread over an adjacent sign is $462 \mathrm{~ms}$, and contains 1.36 syllables (both milliseconds and syllables are averaged over the average lengths per speaker). Mouthings that do spread over adjacent signs have an average length of $673 \mathrm{~ms}$ and 1.64 syllables (again, averaged over the average lengths per speaker). A $t$-test for paired samples shows that this difference is significant both for milliseconds and number of syllables: $t(44)=15.075, p<.000$ with spreadings being longer in milliseconds, and $t(44)=6.056, p<.000$ with spreading mouthings having more syllables than non-spreading mouthings. (The $t$-tests were conducted excluding the one signer who did not spread any mouthings, and therefore had no average spreading.)

In the whole sample of 5,929 mouthings there are 3,901 (65.8\%) mouthings that consist of only one syllable, such as naam ('name') or doof ('deaf'). This includes mouthings that are inflected for number (such as vraag( $t$ ) ('ask') or weet ('know') for singular) and mouthings that have undergone temporal reduction, such as dok from dokter ('doctor') or groon from Groningen, a Dutch city. Temporal reduction is also found in longer spreadings, such as vresel from vreselijk ('terrible') or slechtho from slechthorend ('hard-of-hearing').

In the spreading part of the sample, 444 out of 810 mouthings (54.8\%) consist of one syllable. So while we do find that spreadings on average contain significantly more syllables, a small majority of spreadings still consist of only one syllable. The number of syllables, therefore, cannot explain why mouthings spread, because that would leave the large number of one-syllable spreading unexplained. Further, just as for spreading over multiple signs and regressive spreading, we did not find any salient differences between the set of Dutch words that occur as non-spreading mouthings and the set of words that occur as spreadings (i.e. there are no inherent features of the 
Dutch words that cause them to spread). In the following section we will consider another possible explanation for the occurrence of spreadings, namely the length of the source signs.

\subsubsection{Length of signs}

If mouthings are not particularly long when they spread, is it perhaps the case that the source sign of a mouthing is particularly short, causing the mouthing to overflow to the next manual item? To answer this question, it is necessary to establish the source sign of each mouthing. The Corpus NGT is organised so that annotations for each articulator are independently time-aligned with the video, on different tiers in the ELAN annotation file. Therefore, it is difficult to establish the mutual relations between articulators - in other words, there is no immediate and unequivocal way we can filter out the manual sign that is the source sign for a mouthing. We established the source signs of spreading mouthings by finding all left and right hand gloss annotations that overlapped spreading mouthing annotations, and then took the first gloss on the manual tier as the source sign (or the last gloss on the manual tier in case of regressive spreading). The average length of the signs that are the source of mouthings that spread is $275 \mathrm{~ms}$.

To compare this with the average length of a sign that comes with a mouthing is not a straightforward task. The easiest comparison to make is with the average length of a gloss annotation. In our sample this is $398 \mathrm{~ms}$, but this includes all glosses, also those of signs that do not co-occur with a mouthing or a mouth gesture. And as signs are sometimes held for many seconds, that number may be unrealistically high. Ideally, we would like to compare the average length of a source sign with the average length of a sign that co-occurs with a non-spreading mouthing. But when we extract the latter from our corpus, we get numerous false positives of mouthings that originate in neighbouring signs but overlap with signs that do not have their own mouthing. Since this overlap is only for one or two videoframes the mouthings do not actually spread over that sign (just as in section 3.2.3), rendering a false positive. The solution was to only include signs that occurred with a mouthing having the same annotation value as that sign. This way SCHOOL with the mouthing school was included, as was DEAF (annotated in our corpus as DOOF) with doof ('deaf'). By consequence, mismatching sign/mouthing combinations like THERE with the mouthing Amsterdam, or GROUP with the mouthing klas ('class'), were excluded. This leaves us with 2,643 signs: 1,966 signs with a non-spreading mouthing, and 677 
signs with a spreading mouthing. Averaged over average length per signer, the signs that occur with non-spreading mouthings are $347 \mathrm{~ms}$ in length, and the signs that occur with mouthings that spread over adjacent signs are $282 \mathrm{~ms}$ in length, significantly shorter: $t(44)=5.393, p<.000$ with source signs of spreadings being shorter than signs co-occurring with non-spreading mouthings.

\subsection{Discussion}

\subsubsection{Direction and scope of spreadings}

First, we will compare the numbers of mouthings we found to the total number of mouth actions in our corpus: we found 5,929 mouthings on a total of 7,979 mouth actions, or $74 \%$ of all mouth actions. This sharply contrasts with the NGT data in Crasborn, Van der Kooij et al. (2008), who found that only 39\% of their mouth actions consisted of mouthings. The most likely reason for this is that they only used a narrative register, whereas we used a mixture of narrative and conversational registers. Due to the history of our annotation work, there is an emphasis on the conversational register in our sample (88\%, against $12 \%$ in a narrative register). As Van de Sande and Crasborn (2009) suggested, register is a significant factor in the use of various types of mouth actions. Mouth gestures often have an expressive quality, and may more easily be yielded in storytelling (and thus a narrative register), whereas mouthings are often informative, and thus more suited for interaction (see also Ebbinghaus \& Heßmann, 2001).

The first question we posed was to what extent we find spreading of mouthings over neighbouring signs in NGT. We found that the general conclusion of Crasborn, Van der Kooij et al. (2008) holds for the much larger data set from the Corpus NGT we investigated: spreadings are produced by the large majority of the 46 signers in our sample, it is essentially a rightward phenomenon and usually spans one adjacent sign. The extent to which we found spreadings is similar to that in Crasborn, Van der Kooij et al. (2008). In percentages, they found between $9.8 \%$ and $14.4 \%$ spreadings (dependent on age and register) where we found $13.7 \%$. It is thus clear that spreading is fundamental to everyday sign language use in NGT, and not restricted to an occasional signer using a particular register. 
We then investigated the frequencies of occurrence, direction and scope of spreading, and the inclusion of PTs. We found that all phenomena are omnipresent in the data, also on the level of the individual signer. Leftward spreading or spreading over multiple signs is by no means an idiosyncratic phenomenon. No effects were found for age, region, gender or being a native signer. The variation between the individual signers is substantial: the percentage of spreadings varies between $0 \%$ (the one signer for whom only 19 mouth actions were annotated) and 22.7\%. Given this variability, it is possible that an effect for specific social and/or other variables will be found in a (much) larger sample of signers, but given our results it seems safe to conclude that these effects will not be substantial.

Summing up, we found no obvious sociolinguistic factors that can explain why mouthings spread as they do. All signers use mouthings, and all signers spread a portion of those over one or more adjacent signs, with a small proportion (5\%) spreading regressively so that a mouthing starts at a moment when the hands are still busy with another sign. The variation in frequency is largely a signer-bound phenomenon.

\subsubsection{Spreading over PT and relative length of spreadings}

The second main question related to the timing of spreadings. Is there a preference for mouthings to spread over prosodically light elements like clitics, or does it occur over heavier elements as well? We found that $58 \%$ of all spreadings do so over a PT, and that there is a very high correlation between spreading in general on the one hand, and spreading over PTs on the other.

PTs are an easy target for mouthings to spread over, because they do not have lexical content themselves. As Sutton-Spence (2007: 152) put it: "Deictic pronouns have no need for a mouthing to specify their meaning, because the referent is either present during the utterance or has been identified in the previous sign". On the other hand, it is possible that mouthings may put an unwanted emphasis on the meaning of a sign. A point towards a third person who is present could mean 'John over there'. Without a mouthing, a signer knows what it means, but adding a mouthing like 'John' or 'there' might emphasise a part of the meaning a bit and thus have the unintended effect of altering the communicative intent.

Finally, we wanted to know whether spreading would be merely an articulatory coincidence, a problem in aligning articulations of different lengths, where longer mouthings may push themselves over the end boundary of a short sign to spread 
over the next sign. Mouthings that spread over adjacent signs turned out to be significantly longer than mouthings that did not, both when measured in syllables as well as in milliseconds. But since single-syllable mouthings also frequently spread, syllable length cannot be the only explanation for the spreading behaviour. The length in milliseconds, however, can give us an indication to the possibility of it being one of the factors involved. We found a substantial correlation between the lengths of spreading and non-spreading mouthings on the signer level, meaning that there is a signer-independent relation between the length of non-spreading mouthings and the length of spreading mouthings. The important conclusion here is that, on average, spreading mouthings do take up more time than non-spreading mouthings. The question remains: does this happen because the signs are short, or because the mouthings are long? In other words: are mouthings altered to match the hands, making the hands the head of the mouth (Boyes Braem \& Sutton-Spence, 2001)? We have not looked in detail at the Dutch words that spread over adjacent signs, but we have no reason to believe that they are different from non-spreading mouthings. Although we do not have detailed numbers as yet, there are numerous examples in our data set of Dutch words that appear both in spreading mouthings as in non-spreading mouthings. Taken together, these findings suggest that spreading mouthings are not incidents of motor planning of different articulators. This leaves open the possibility that indeed spreading mouthings serve to demarcate short prosodic domains, tying together manual signs that morphosyntactically belong together, just as Sandler (1999) first proposed for ISL.

\subsection{Conclusion}

We replicated the results of Crasborn, Van der Kooij et al. (2008) with a much larger corpus of 46 participants in an everyday language use setting. We found that both the use of mouthings and the spreading of mouthings over adjacent signs are not idiosyncratic phenomena: all signers do it, in all sorts of combinations of signs and mouthings. Clearly, it is a fundamental characteristic of everyday sign language use in NGT. Spreading mouthings are not accidental. In a corpus study like the present one, one is dependent on the corpus metadata to be able to discover patterns that are related to properties such as age of acquisition. For the Corpus NGT, limited information was available on the details of language development for each signer, for both the signed 
and the spoken language. The available information on deafness in the immediate family (see section 3.2.2 above) is not necessarily very informative about the relative quantity of language input to the child in the signed and the spoken language. For the creation of new corpora, we recommend this as an important point of attention that may benefit all studies based on the corpus.

Now that we showed the omnipresence of spreading, further research is needed to seek explanations as for why spreading occurs. Sandler (1999) proposed the prosodic word as the outcome of a cliticisation process, and found supporting evidence in the spreading of mouthings. Does spreading match other prosodic cues such as spreading of the non-dominant hand? To what extent do the rhythmic structure of both signs and mouthings influence each other? A detailed analysis of the morphosyntactic and prosodic context is needed to provide answers to these questions. Further, psycholinguistic and neurolinguistic studies could involve experiments to clarify the mechanisms underpinning the spreading of mouthings: to what extent are they linked in the mental lexicon and to what extent can they be linked ad hoc in the production of new sentences? A final interesting question is why PTs are sometimes cliticised with the preceding sign and its spreading mouthing, but at other times are pronounced as independent signs, without a mouth action or sometimes even with their own mouthings. Answering this may further clarify the prosodic structure of signed languages and the way a sign language mixes with the surrounding spoken language. Our knowledge about this type of code-mixing will help us to better understand the language acquisition process of both the signed and the spoken language in bimodal bilinguals, whether in first or second language acquisition (see e.g. Baker \& Van den Bogaerde, 2008). 


\section{Chapter 4: The prominence of spoken language elements in a sign language}

Slightly adapted from: Bank, R., Crasborn, O., \& Van Hout, R. (under review). The prominence of spoken language elements in a sign language.

\subsection{Introduction}

ilingual speakers' language use has been characterized in terms of a variety of possible language contact phenomena, sometimes showing a dense integration of elements from different languages (see Muysken, 2013, for an overview). Deaf and hearing users of signed languages form a special case in the study of bilingualism and language contact, as both languages can be realized simultaneously: spoken language by the oral articulators (with voice or without) and sign language by other visible articulators. Although lexical content is mainly conveyed by the hands in all sign languages, other articulators play an essential role as well, such as eye gaze, position of head and torso, and mouth actions (see Crasborn, 2006, for an overview). These mouth actions may be divided into mouth gestures and mouthings, the former being sign language inherent, the latter originating from the spoken language of the hearing community in which a deaf community is embedded (see the various contributions to Boyes Braem \& Sutton-Spence, 2001). They are 
(usually silently) mouthed words from the surrounding spoken language, or parts thereof; typically, but not always, the semantics of the spoken word overlaps with that of the manual sign (Bank, Crasborn, \& Van Hout, 2011). In Sign Language of the Netherlands (Nederlandse Gebarentaal, NGT), virtually all signers use spoken language mouthings with their signing (Bank et al., 2011; Schermer, 1990). This is in fact the case in many signed languages studied to date (e.g. Boyes Braem, 2001, for Swiss-German Sign Language; Crasborn, Van der Kooij, Waters, Woll, \& Mesch, 2008, for British, Dutch, and Swedish Sign Languages; Ebbinghaus \& Heßmann, 1994, for German Sign Language; Mohr, 2012, for Irish Sign Language; Nadolske \& Rosenstock, 2007, for American Sign Language; Nyst, 2007, for Adamorobe Sign Language; Schuit, 2012, for Inuktitut Sign Language; Sutton-Spence, 2007, for British Sign Language; Sze, Woodward, Wijaya, Satryawan, Isma, \& Suwiryo, 2013, for Jakarta Sign language; Vogt-Svendsen, 2001, for Norwegian Sign Language). This long list makes clear that signers around the world use a combination of the language most accessible to them (i.e. a signed language) and the language used by most people in their surroundings (i.e. a spoken language). However, the effects of language contact in the case of signed and spoken languages remains largely unexplored. For most sign languages mentioned above, studies were conducted with only a few signers, in laboratory settings, or were focused on a general description of the language instead of studying language contact. Moreover, cross-language activation studies often focus on perception instead of production (e.g. Morford, Wilkinson, Villwock, Piñar \& Kroll, 2011) or only describe hearing bimodal bilinguals (Emmorey, Borinstein, Thompson, \& Gollan, 2008; Emmorey, Petrich, \& Gollan, 2012).

The question whether signed languages can exist without mouthings stemming from the surrounding spoken language is an academic one. Schermer (1985) suggested earlier that "the existence of a pure sign language, without the occurrence of any speech, among deaf adults, is more or less a theoretical construct" (Schermer, 1985: 288). Since there is no Deaf country or other place only inhabited with monolingual deaf signers, there is no way we could witness how a signed language would develop without any influences from outside. One may hypothesize that the occurrence of mouthings is related to amount of education in the spoken language. The only case of a sign language without mouthings that we are aware of is Kata Kolok in Indonesia, a rural sign language in which "there are virtually no mouthings" (De Vos \& Zeshan, 2012: 17). Indeed for the signers in this village deaf education is a very recent phenomenon, but at the same time the amount of schooling for deaf users 
of the languages listed above is highly variable and still they appear to incorporate mouthings in the sign language on a substantial scale. Kata Kolok remains an exception, it appears.

One of the questions sign language researchers are pursuing concerns the linguistic status of mouthings in sign language. Some consider mouthings to be an inextricable, inherent part of the sign language lexicon (e.g. Boyes Braem, 2001; Sutton-Spence \& Day, 2001), yet others argue that, while relevant for communication, mouthings should not be regarded as part of the lexicon (Ebbinghaus \& Heßmann, 2001). In support of the latter view, Vinson, Thompson, Skinner, Fox, and Vigliocco (2010) showed with picture-naming tasks and word-translation tasks that signs and mouthings are represented and accessed largely independently from each other.

Code-mixing by unimodal bilinguals (Muysken, 2000) is a sequential phenomenon by nature of the modality. To describe the bimodal code-mixing that occurs with hearing bimodal bilinguals (e.g. CODAs, children of deaf adults), Emmorey, Borinstein, and Thompson (2005; also Emmorey, Borinstein, Thompson, \& Gollan, 2008) proposed the term 'code-blending'. Van den Bogaerde and Baker (2005; also Baker \& Van den Bogaerde, 2008) adopted this term to also cover the bimodal bilingual input to children from their deaf mothers, and the output from both deaf and hearing children to their deaf mothers. Since virtually all deaf signers in developed countries are functionally bilingual - as a result of growing up in a hearing world and receiving education in (at least) spoken language (cf. Ann, 2001; Lucas \& Valli, 1992) - we follow Van den Bogaerde and Baker (2005; Baker \& Van den Bogaerde, 2008) and use the term code-blending for deaf signers using mouthings.

The present paper aims to answer the question as to the independence of sign language from spoken language: to what extent does spoken Dutch play a role in conversations between deaf people whose primary language is sign language, both in terms of order of acquisition and in terms of proficiency? Browsing the video recordings of the Corpus NGT (Crasborn \& Zwitserlood, 2008b; Crasborn, Zwitserlood, \& Ros, 2008), one gets the strong impression that there is a lot of variation between individual signers, both in their style of articulation of manual signs and in the style of using non-manual features such as mouthings.

Variation in signing between individual learners may be related to social distinctions. The body of research into the sociolinguistics of signed languages is steadily growing, most notably through the work of Ceil Lucas (e.g. Lucas, 1995, 2001; 
Lucas \& Valli, 1992). In Lucas, Bayley, and Valli (2001), the authors report that the social constraints that influence variation in American Sign Language (ASL) are the same as the ones that influence variation in spoken language: age, gender, ethnicity, social class and region. In the domain of manual phonology, for instance, they find regional differences for handshape, and age differences and social class differences in the use of a specific handshape variant of a sign. They further report on gender variation in the use of overt pronouns. Similar sociolinguistic studies have been conducted for other sign languages, like for example British Sign Language (BSL; Fenlon, Schembri, Rentelis, \& Cormier, 2013; Sutton-Spence \& Woll, 1999).

When the language of an age group is contrasted with that of an older or younger adult group, differences are expected to occur, thus reflecting diachronic language change (Kerswill, 1996). In the case of signed languages, educational reforms are expected to have a major impact on diachronic language change due to the fact that for many deaf children with hearing parents, most sign language input is available in deaf schools. In the course of the last century, the language policies of deaf schools have varied considerably, showing an overall development from oralist education to more inclusion of sign language in the curriculum. At the same time, language policies have varied from school to school. Both according to the literature and to informal accounts of deaf people, even in the most oralist schools, there was plenty of sign language use outside the classrooms in the breaks and after school. The impact of special education policies on a sign language is well documented for Ireland, where strict separation of boys and girls has led to many gender differences in the language (LeMaster, 1990, 2000; LeMaster \& Dwyer, 1991).

Studies on variation in the use of mouthings, however, are scarce. This may not be surprising, since most sign language research is done on ASL, a language that has a longstanding reputation of hardly featuring any mouthings - but see Nadolske and Rosenstock (2007), who show that "contrary to what has been claimed in the literature, mouthings contribute significantly to the formal and semantic aspects of ASL" (2007: 35). Preliminary work on BSL found no differences between groups of different backgrounds (Sutton-Spence \& Day, 2001). Regarding differences between age groups, Hoyer (2004) mentions in passing that mouthings are more frequently used by elderly signers in Finnish Sign Language. However, she does not refer to empirical evidence for this claim. Mohr (2012) finds both gender differences and age differences in a study on mouthings in Irish Sign Language (ISL), which may not be surprising in the light of the overall gender differences in ISL lexicon referred to above. 
In Mohr's study, women on average use considerably more mouthings than men. When split into age groups, the number of mouthings by women turns out to be quite constant, whereas the use of mouthings by men decreases over age groups. Younger male signers use more mouthings than older male signers. In the youngest age group, males and females are comparable in their use of mouthings. Mohr ascribes the Irish situation to the separate institutions for deaf boys and girls in Ireland: oralism was introduced at a later stage to the boys' school (1957) than to the girls' school (1946), thus accounting for the lower numbers of mouthings by elderly men. Regarding any recent changes in language policy away from oralism, Mohr mentions that "today, the schools' current language policy states that teachers should communicate with the children in whatever language is most suitable to their needs, be it Irish Sign Language, Signed English or spoken English" (Mohr, 2012: 51), a change in policy that started to take shape during the 1980s (Leeson \& Saeed, 2012).

In a study on NGT, Van de Sande and Crasborn (2009) looked at the proportions of mouthings and mouth gestures between registers (narrative vs. interactive) and between early and late learners, where they classify late learners as those who start sign language acquisition at around 3 years of age, with a mean of 4.5 years in their sample. They found a significant difference between registers, with more use of mouthing in interactive registers. Regarding early and late learners, they found a tendency towards more frequent use of mouthings by later learners, although this was not significant. It should be noted that their division of their sample into early and late learners largely coincides with the age groups that we will be distinguishing in the study reported below. In a study on ASL, Nadolske and Rosenstock (2007) found a similar register effect. Stadthaus (2010) used the same sample as Van de Sande and Crasborn (2009), consisting of signers from the Amsterdam and Groningen regions, as well as a few signers labelled 'Other Region'. She looked at jaw drop in mouthings in NGT, operationalising the hypothesis that it may not be the number of mouthings in relation to manual signs that varies but rather the way they are articulated that results in the impression that older signers use more mouthings than younger signers: speech and/or mouthings of older signers may be less sloppy because of stern speech therapy in oralist times. However, she did not find any differences between the age groups. Finally, Bank, Crasborn, and Van Hout (2013), looking at spreading of mouthings over adjacent signs, found no differences in spreading behaviour between older and younger signers. 
Mouth actions can vary between individuals or groups in several possible ways. First, there can be variation in the number of mouthings relative to the number of signs. Although Van de Sande and Crasborn (2009) found no differences between age groups, it may be that their sample was just too small. In the present study we therefore investigate frequency differences based on a much larger sample.

Second, there may be variation in articulation. Stadthaus (2010) came up with a system to score jaw drop, or amplitude of articulation (see above). While it seems like a good idea to annotate the entire corpus for properties like amplitude or precision of articulation, unfortunately time did not permit us to do so for a substantial data set, and we discarded this as a possible feature for the present study.

Third, it may be the case that older signers indeed do not use more or more pronounced mouthings, but that in cases when they do not know a sign or when they want to be efficient in terms of manual articulation, they simply point at a location in signing space while mouthing the intended spoken word. This would explain why previous research did not find differences in manual sign to mouth action ratios for different age groups, but would still add to the impression of older people using more mouthings. This effect did not show up in the Bank, Crasborn, and Van Hout (2011) study on variation in highly frequent signs, because they left pointing signs out of their study.

Finally, there may be variation between groups in their use of mouthings as compared to mouth gestures. Bank et al. (2011) found variation in the choice between mouthings and mouth gestures, but did not look into variation of that choice over groups. That is, older signers may be more consistent in their mouthings, while younger signers may show more variation in their choices.

In this paper, we will seek answers to the following research questions. To begin with, how prominent is the use of code-blends exactly in NGT in terms of frequency? Further, are mouthing frequencies different when comparing age, gender, region, level of education or having deaf or hearing parents? And finally, could it be that some groups more often combine mouthings with pointing signs than with content signs? 


\subsection{Methodology}

We analysed parts of the Corpus NGT (Crasborn \& Zwitserlood, 2008b; Crasborn, Zwitserlood, \& Ros, 2008). This corpus contains video data of 92 prelingually deaf signers, recorded in pairs, who retell video clips and picture stories, and discuss issues related to deafness, education and sign language. The signers in the Corpus NGT were selected on the base of signing skill, not their knowledge of the Dutch spoken language. Still, all participants have followed secondary education where they successfully completed exams in spoken Dutch.

Annotations in the corpus are made on a variety of tiers for the different articulators. For manual components, there are separate, independent tiers for the left and right hands, ensuring an accurate annotation of what each hand does. This benefits, for instance, research on handedness, or spreading of the non-dominant hand (e.g. Sáfár \& Crasborn, 2013), but in an automated analysis it is not immediately clear whether two co-occurring manual glosses actually comprise one two-handed sign. Since the Corpus NGT reflects everyday language use, signers may deviate from citation forms (by articulating one-handed signs as two-handed and vice versa), use their non-dominant hands as a buoy, or articulate two one-handed signs simultaneously. This makes determining the number of signs currently in the corpus a non-trivial task.

In the past, annotation work for manual components has concentrated on signers from the Amsterdam and Groningen regions, and therefore mouth annotations are concentrated in this part of the corpus as well. Further, for most signers only clips in the conversational register (discussions) are currently annotated, there are just a few signers for whom data are available in both conversational and narrative registers (retelling stories). Van de Sande and Crasborn (2009) showed that there is a significant effect for register in the use of mouthings, with more mouthings being used in a conversational register. With not much narrative data at our disposal we decided to concentrate on the conversational register only, reflecting everyday language use.

For some previous studies, mouth annotation work was only done relating to specific signs throughout the corpus, while other studies resulted in clips fully annotated for the mouth. For the current study, we annotated mouth actions for many clips that had been partially annotated before, thus resulting in a more coherently annotated corpus. There are 257 clips that contain conversational data and that are at least partly annotated for mouth actions, containing data from 75 signers from all five 
regions in the Netherlands. Five signers with less than ten mouth annotations each were excluded from our analyses. All results for this sample (Sample 1) are thus based on 70 signers from 252 clips. We divided the sample into three age groups: 17-26, 27-40 and 41-84. To see whether there would be an effect in highest form of education that participants had followed, we distributed the signers over five groups: primary and secondary education, and three types of vocational education common in the Netherlands: lower, middle and higher vocational education (LBO, MBO and HBO, respectively).

We will be looking at several sociolinguistic variables to see whether variation in mouthings occurs: region, gender, age, highest level of education and whether the child has deaf parents or not (which we will be calling nativeness). Signed languages are a special case in that respect because the L 1 of a child's parents will not necessarily become the L1 of the child (as most deaf children have hearing parents). Moreover, it is often hard to tell whether the signed language is the L1 or the L2, since they are often learned at the same time as the spoken language. There are very few native signers if you consider a native signer to be someone who learns sign language right from birth from fluently signing parents. Only a small minority of deaf born children (usually estimated between 5 and 10\%) have deaf parents (Mitchell \& Karchmer, 2004), of whom, in turn, only a small minority would have been real native signers themselves.

To investigate the question of mouthings co-occurring with pointing signs, we could only use clips that were fully annotated for mouth actions, because for every pointing sign we wanted to see what kind of mouthing co-occurred with it, including 'no mouth action on pointing signs'. If we would have been using partly annotated clips, we would not have been able to distinguish between 'no mouth action' or 'not annotated yet' in an automated analysis. This subset of Sample 1 (that we will dub Sample 2) consists of 38 clips fully annotated for hands and mouth, spanning 86 minutes. There are, coincidentally, 38 signers in this sample, all from the Amsterdam and Groningen areas.

To get an idea of how often mouth actions actually happen, we calculated the ratios between signs and mouth actions and between signs and mouthings, using Sample 2 (because, again, we had to be sure that all mouth actions in a clip would be annotated). Since the gloss annotations in the Corpus NGT do not easily allow for the count of lexical items (see above on the separate annotation of the left and right hand), we counted all right-handed and left-handed glosses, and assumed that, with one- 
handed signs, all right-handed signers are strictly right-handed, and all left-handed signers are strictly left-handed (thus disregarding dominance reversals, see Crasborn \& Sáfár, in preparation). Thus, for right-handed signers, the number of glosses for the left hand illustrates the number of two-handed signs, and the number of glosses for the right hand then illustrates both the number of two-handed signs and the number of one-handed signs. Ambidextrous signers were left aside (see Sáfár, in preparation, on how to determine handedness in an automated way). We realize this is an oversimplification, but it will give us a general idea about the number of oneand two-handed signs in the context of mouth actions.

\subsection{Results}

\subsubsection{Results on mouth action frequency as compared to manual signs}

There are 11,905 glosses for both hands for all 38 signers in Sample 2. The 32 righthanders account for 10,265 glosses: 7,665 for the right hand and 2,600 by the left hand, so by our rough estimates the right-handed signers make 7,665 signs (of which 2,600 (34\%) are two-handed signs as indicated by the number of glosses for the left hand, and therefore 5,056 (66\%) are one-handed signs). The four left-handers account for exactly 1000 glosses: 721 for the left hand and 279 for the right (so 721 signs, of which $39 \%$ two-handed and $61 \%$ one-handed). In sum, discarding the two ambidextrous signers for now, 36 signers make 8,386 signs (66\% one-handed and 34\% two-handed). This comes very close to what Sáfár (in preparation) found in a study of handedness in NGT. Using a different subset of the Corpus NGT and balancing right-handed, lefthanded and ambidextrous signers, she found $67.7 \%$ one-handed signs against $32.3 \%$ two-handed signs. Moreover, she remarks that there was considerable variation between participants in the use of different sign types, which likely contributes to the differences between her percentages and ours. Note, moreover, that these are figures that come from language use; in a phonological study on the NGT lexicon citation forms, Van der Kooij (2002) reports that 53.5\% of 3,084 signs are one-handed, and $46.5 \%$ two-handed. All in all, we can safely presume that our estimation is good enough to work with.

Zooming in on the use of mouthings, we found that our 36 left and righthanders with their 8,386 signs also produce 6,125 mouth actions, comprising 5,106 
(83.4\%) mouthings and $870(14.2 \%)$ mouth gestures. In the remaining 149 cases it was unclear what the type of mouth action was. This makes the ratio of mouth actions to manual signs 0.73 , and the ratio of mouthings to manual signs 0.61 . Please note that these figures only tell us something about the numbers of discrete mouth actions and mouthings compared to manual signs, not how long they last. To get the ratios for duration we again took only the right hand glosses of the right-handed signers and the left hand glosses of the left-handers, added up the durations of these glosses and also added up the durations of the signers' mouth actions and mouthings. The sum of all manual gloss durations is 2,994 seconds, the sum of all mouth action durations is 3,144 seconds, and the sum of all mouthing durations is 2,419 seconds. Thus, looking at length, the ratio of mouthing to sign duration is 0.81 , and the ratio of mouth action to sign duration is even 1.05 .

The ratios of durations of mouthing and mouth actions compared to manual signs show that the mouth hardly ever stands still, and that there is a continuous stream of linguistic information both on the hands and on the mouth. Moreover, in a given signing stream in NGT, more than $80 \%$ of the time Dutch words are mouthed, providing an almost continuous mix of two languages.

\subsubsection{Results on mouthing frequencies for various sociolinguistic variables}

Our main Sample 1 (70 signers, 252 clips at least partly annotated for mouth actions) contains 10,814 mouth actions. Of those, 9,120 (84.3\%) are mouthings and 1,463 (13.5\%) are mouth gestures. The remaining 231 were either not visible or undecided. Figure 4.1 shows the outcomes for the mean percentages of mouthings when the data are split up for the five sociolinguistic variables.

One of the most striking results that can be seen in Figure 4.1 is the large percentage of mouthings: there are no groups where the average number of mouthings per 100 mouth actions is lower than 80 . Further, it is evident that the differences between the subgroups are small, the biggest difference being between 80 en $100 \%$ for region.

It should be noted, however, that the few signers from the Voorburg, Rotterdam and Sint-Michielsgestel regions - i.e. the three highest bars in the graph are represented with only a small amount of annotated data: these 11 signers account for only 274 mouth actions, a mere $2.5 \%$ of the total amount. 


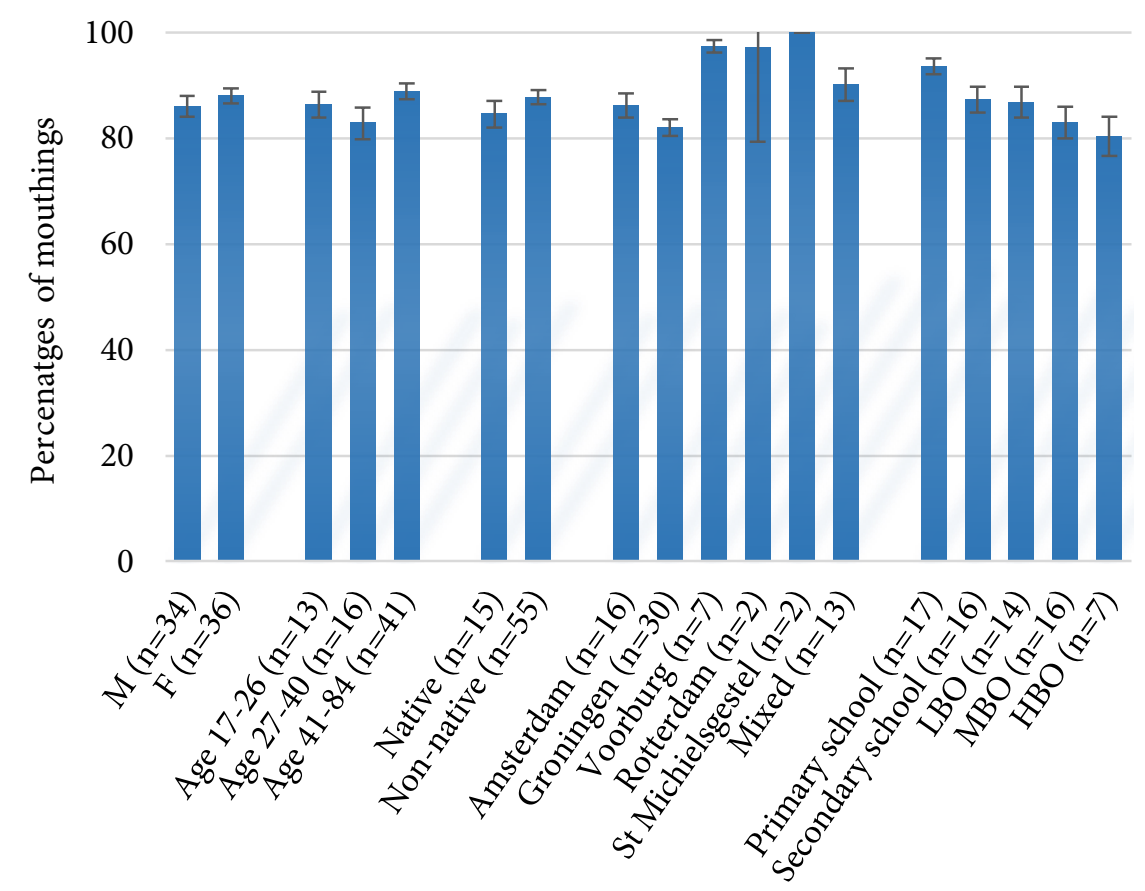

Figure 4.1. Percentages of mouthings, for gender, age, nativeness, region, and highest level of education. Error bars have the value of two standard errors.

Figure 4.1 effectively shows how small the differences are between groups, but does not reflect the differences within the groups. The scatter plot in Figure 4.2 visualizes how the proportion of mouthings to mouth actions is spread across age, gender and native signers, including the weight of the subject in terms of numbers of annotations.

Again, one of the most striking results is the high percentage of mouthings: the lowest percentage of mouthings is $64.6 \%$ of all mouth actions. There is a high score for mouthings, but the variation between participants is large. Further, it can be seen that no distinctive groups can be formed within gender, nativeness or age. There are a few signers with a mouthing to mouth action ratio of nearly 1.00 that contribute less than 100 mouth actions to the sample; while it is possible that this high score can be ascribed to the low amount of annotated data available, there are also signers that do contribute a high number of mouth actions and still score almost a $100 \%$ for mouthings (such as $98.4 \%$ for a 61 -year old non-native male, contributing 369 mouth actions to the sample). 


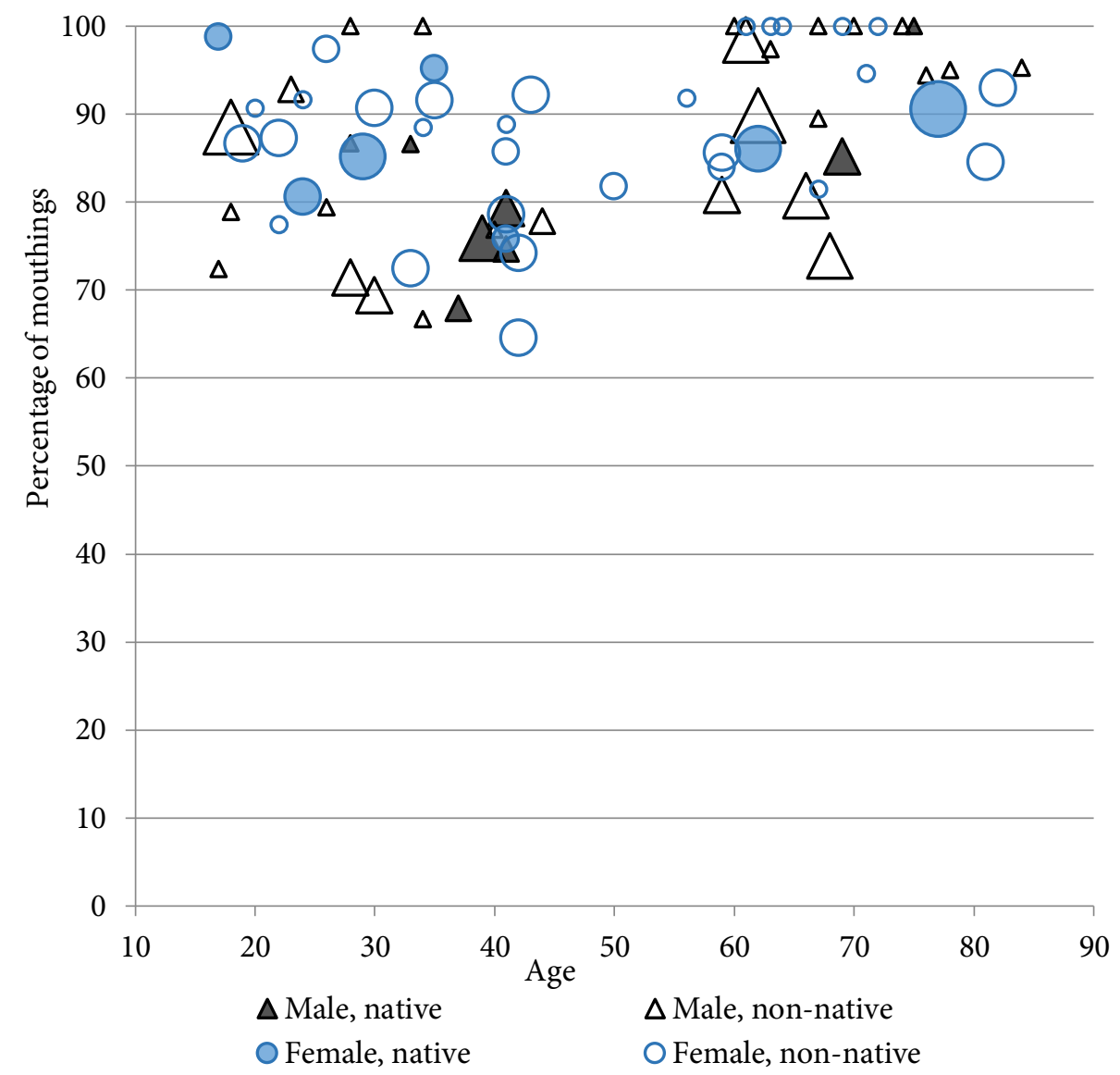

Figure 4.2. Scatter plot for percentage of mouthings as a function of age. Black triangles represent male signers, blue circles represent female signers; solid marks represent native signers, outlined marks represent non-native signers. The size of a mark represents the number of tokens for that signer, in five categories (10-100, 101-200, 201-300, 301-400 or more than 400 tokens).

We investigated the effect of the set of sociolinguistic variables by applying analysis of variance. No effects were found for gender $(\mathrm{F}(1,68)=.678, \mathrm{p}=.413)$, age $(\mathrm{F}(2,67)=$ $2.304, \mathrm{p}=.108)$, and nativeness $(\mathrm{F}(1,68)=1.201, \mathrm{p}=.277)$. Significant effects were found for region $(\mathrm{F}(5,64)=6.525, \mathrm{p}=.000)$ in that signers from Voorburg use more mouthings, and for education $(\mathrm{F}(4,65)=3.931, \mathrm{p}=.008)$ in that the use of mouthings decreases with better education (post-hoc analysis, excluding Rotterdam, Sint Michielsgestel and Mixed regions). 


\subsubsection{Results for combinations of mouth actions and pointings}

In the continuously annotated Sample 2 (38 signers, 38 clips, 86 minutes), there are 1,758 annotations for points, including 982 annotations (55.9\%) for PT (i.e. pointing somewhere in the signing space, not to a present referent), 703 annotations (40.0\%) for PT:1 (i.e. point to self) and 73 (4.2\%) other forms (such as PT:B for a downward point (in Dutch: beneden), or PT:W for a point towards the other index finger (in Dutch: wijsvinger), and a few others).

There are several observations to be made when it comes to mouth actions accompanying pointing signs. The total of 1,758 PT annotations includes 476 PT annotations (27.1\%) that co-occur with one mouth action; 336 (70.6\%) of those are mouthings and $140(29.4 \%)$ are mouth gestures. We thus find a much lower percentage of mouthings for this category than the $84.3 \%$ average for all signs as displayed in Figure 4.1. Further, there are 548 PT annotations (31.2\%) that co-occur with a mouth action that is also present with one of the adjacent signs (thus, a spreading mouth action); 433 of those are mouthings, 115 are mouth gestures. Then, there are 96 PT annotations (5.5\%) that co-occur with a mouth action that accompanies a sign on the other (dominant) hand; finally, there are 638 PT annotations (36.3\%) that do not co-occur with any mouth action. No significant differences in ratios of PT annotations with or without various types of mouth actions were found for age, region, gender, or having deaf parents or not. There was a significant effect for education level, but only for the combination of a PT co-occurring with one mouth action $(F(4,20)=3.503, p=.018)$. When we split this category into its subcategories mouthings and mouth gestures, the effect lies with mouthings $(\mathrm{F}(4,30)=4.048, p=.010)$. Table 4.1 summarizes and Figure 4.3 visualizes these findings. 
80 | Chapter 4: The prominence of spoken language elements in a sign language

Table 4.1. Combinations of co-occurrence of pointing signs (PT) and mouth actions.

\begin{tabular}{|c|c|c|c|}
\hline \multirow{3}{*}{$\begin{array}{l}\text { All PT annotations } \\
\text { PT co-occurs with only one } \\
\text { mouth action }\end{array}$} & \multicolumn{3}{|l|}{$1758(100 \%)$} \\
\hline & $476(27.1 \%)$ & & $(100 \%)$ \\
\hline & & Mouthing & $336(70.6 \%)$ \\
\hline \multirow{4}{*}{$\begin{array}{l}\text { PT co-occurs with mouth action } \\
\text { that spreads over multiple signs }\end{array}$} & & Mouth gesture & $140(29.4 \%)$ \\
\hline & $548(31.2 \%)$ & & $(100 \%)$ \\
\hline & & $\begin{array}{l}\text { Spread from } \\
\text { mouthing }\end{array}$ & $433(79.0 \%)$ \\
\hline & & $\begin{array}{l}\text { Spread with } \\
\text { mouth gesture }\end{array}$ & $115(21.0 \%)$ \\
\hline PT is on nondominant hand & $96(5.5 \%)$ & & \\
\hline No mouth action & $638(36.3 \%)$ & & \\
\hline
\end{tabular}

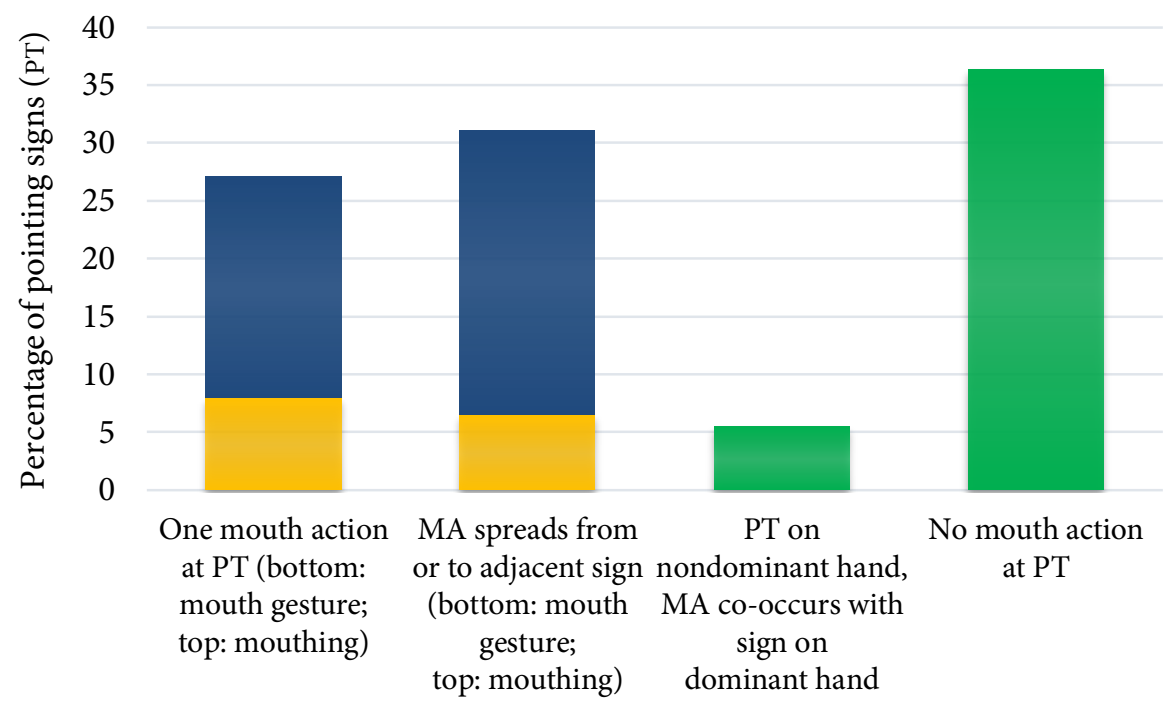

Figure 4.3. Distribution of types of mouth action (MA) occurring with pointing signs (PT).

There were $336 \mathrm{PT}$ annotations that co-occur with only one mouthing. The largest word class present in this segment is that of pronouns (72 tokens, $21.4 \%$ ), mainly consisting of ik ('I', 57 tokens). Other large groups are those of verbs (46 tokens 
(13.7\%), with 20 tokens from the zijn ('to be') paradigm), adverbs (13.1\%), prepositions (11.9\%) and possessives (9.8\%).

A small group of 18 tokens (5.4\%) consisted of constructions with multiple mouthed Dutch words:

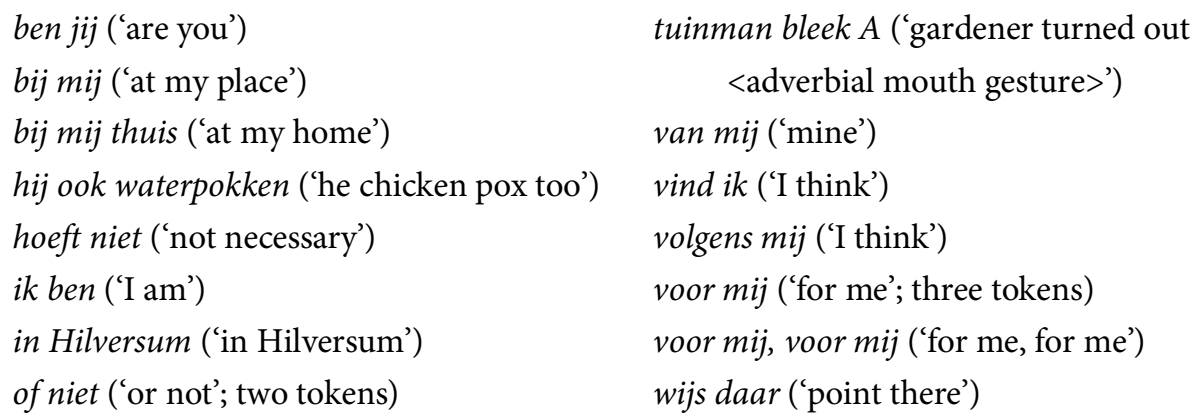

tuinman bleek $A$ ('gardener turned out <adverbial mouth gesture>')

van mij ('mine')

vind ik ('I think')

volgens mij ('I think')

voor mij ('for me'; three tokens)

voor mij, voor mij ('for me, for me')

wijs daar ('point there')

With very few exceptions (hoeft niet; of niet; tuinman bleek <mouth gesture $>$ ), these multi-word mouthings contain a pronoun $(i k, m i j, j i j)$ or a locative expression (thuis, daar).

\subsection{Discussion}

\subsubsection{Proportion of mouth actions and mouthings compared to signs}

We found high numbers of mouthings and mouth gestures compared to signs in our data. When comparing signs with mouthings, we found a mouthing to manual sign ratio of 0.61 for number of mouthings, and a ratio of 0.81 for duration of mouthings. When comparing signs with all mouth actions, we found a mouth action to manual sign ratio of 0.73 for number of mouth actions, and a ratio of 1.05 for duration of mouth actions. The latter ratio shows that in one respect, the mouth is actually more active than the hands. We should point out here that the way we got the number of signs was a kind of rough estimate. However, it is less than two percent point off from dedicated studies (Sáfár, in preparation), so we feel confident about the results we found. This high activity of the mouth as articulator can in part be explained by the fact that our manual sign annotations do not include the transitional movements from 
sign to sign, and between rest position and sign. Compared to the small and rapid movements of the mouth, these transition times take significant amounts of time to perform, not leading to the overall impression that the mouth is active more of the time than the hands.

Bank et al. (2011) looked at variation in mouthings for highly frequent signs, and found that variation lies not so much in the Dutch lexical items that are used, but more in the distribution between mouthings and mouth gestures. The little variation found in that study within the use of mouthings was almost always within the same semantic field (such as excellent instead of good accompanying the sign GOOD). Given the substantially larger number of mouthings in the data set of connected signing that we used in the current study, it is safe to say that there is great semantic redundancy in the use of mouthings in NGT as compared to manual signs. But redundant or not, mouthings do convey linguistic information, and it is therefore of vital importance to include the role of the mouth in sign language research if we want to understand the way deaf communication is organized.

\subsubsection{Mouthing frequencies related to various sociolinguistic variables}

In our analysis of mouthing frequencies we found that all signers have a mouthing to mouth action ratio between 0.65 and 1.0, irrespective of age, gender or nativeness or the number of mouth actions signers contributed to the sample (cf. Figures $4.1 \& 4.2$ ). We found no effect for age, nor for gender or nativeness. These findings contradict our initial thoughts about age differences in mouthing use: unlike in Finnish Sign Language (Hoyer, 2004) there are no age differences in NGT, and unlike in Irish Sign Language (Mohr, 2012) there are no gender differences in NGT. We did find an effect both for region and for highest level of education, with signers from Voorburg using more mouthings than signers from other regions, and with better educated signers using less mouthings. However, it is hard to say which of the two causes the effect, region or level of education: because of an unfortunate concurrence we only have lower educated signers for the Voorburg region in our annotated sample. Adding to that, as we have said above, although the Voorburg signers are represented with 7 participants (10\% of all participants), they account for only $1.9 \%$ of the data. We would like to hypothesize that the region effect is less prominent than the effect of level of education, since the latter is found for the great majority of participants. It should be emphasized that, although higher educated signers use significantly less mouthings than lower educated signers, the highest educated signers still have an 
average of $80 \%$ of mouthings to mouth actions, so we maintain the claim that the production of Dutch words in the form of mostly silent mouthings is a prominent feature of the language. We speculated a priori that the ratio of mouthings to mouth actions would be even higher with higher education, because with education comes a better language proficiency in Dutch, resulting in a larger vocabulary, while the NGT vocabulary may not keep up because there are not many signers in higher education settings. When discussing our results with two deaf informants who both received higher education, they suggested that higher educated signers are better able to separate the two languages, and that they are quicker and more creative to visualize concepts, playing with it to "put it into signs" without mouthings. It is then the creativity that comes with language proficiency that reduces the number of codeblends. If this is indeed the case, then these higher-educated signers do not do it very often, as even for them the mouthing to mouth gesture ratio is very high. Further research would be needed to seek out in what way level of education influences signing, and if there is any difference in the way mouthings and mouth gestures are used depending on proficiency in NGT and in Dutch. It may be the case, for instance, that sign/mouthing combinations are more variable for some signers than for others, or that there is a stronger tendency to combine signs with mouth gestures instead of mouthings for some signers.

\subsubsection{Combinations of mouth actions and pointing signs}

We looked in more detail at the type of mouth actions co-occurring with pointing signs (РT), this being the most frequent ID-gloss in the corpus. We found that there are three types of co-occurring actions that are roughly equally frequent: no mouth action, one mouth action, or a spreading mouth action that is shared with one or more adjacent signs. Both for the one mouth action and spreading mouth action categories we found that in $70.6 \%$ to $79.0 \%$ of those events the mouth action was a mouthing. This comes as no surprise given mouthing ratios we reported above, and is in line with an earlier study (Bank et al., 2013). Just as for the whole data set, for pointing signs we found no effects for age, gender, region or nativeness either; there was an effect for education level in the use of one mouth action per PT, this seems to reflect the effect found for education level that we found for all signs.

One methodological note is in order regarding the present annotation of pointing signs in the Corpus NGT. As we currently use a rather phonetic definition of pointing for the glossing of our pointing signs, lacking a clear analysis of the function 
of these signs, many PT glosses arise from short index finger extensions of which the linguistic function is dubitable at best (cf. Masakata, 2003, for pointing gestures in hearing children). As the index finger has a separate extensor muscle, and the other three fingers have a shared flexor muscle, there are many rest positions and transitional movements in which the index finger extends more than the other fingers (Ann, 1993, 2006). These pointing-like articulations are often hard to distinguish from explicit linguistic articulations on the basis of their phonetic form, as intentional pointing signs are also articulated with a variety of positions of the unselected fingers (Fenlon et al., 2013, for BSL). Also, the duration of linguistic pointing signs is highly variable and may be very brief. All in all, the number of pointing signs in our data set may be overestimated. Conducting an in-depth analysis of these cases would require a detailed morphosyntactic analysis of these cases, and will constitute a study in itself (see also the various studies in Kita, 2003). We surmise that this does not influence the general results of this part of our study, but that it may contribute to the large spread in individual differences that we found.

\subsection{Conclusion}

We showed that the majority of manual signs is accompanied by a mouth action in NGT dialogues, and that the majority of these mouth actions consist of (fragments of) words from spoken Dutch. We further showed that there are no age, gender, region or nativeness-related differences in the use of these mouthings in NGT. A small effect was found for level of education, higher educated signers using fewer mouthings. Future research should explore this effect further, for example by looking at the variation in sign/mouthing combinations or in sign/mouth gesture combinations related to educational level. Are higher educated signers perhaps more skilled in combining different semantic elements from the two languages? Further, perception and recognition studies of sign language need to take into account the overwhelming presence of mouth actions in signed interaction, something that is largely ignored in studies that do not focus exclusively on the mouth. What is the relative role of the two information streams in different stages of the comprehension process?

Our findings also impact our understanding of the cognitive representation of signs and the organization of mental lexicon. Although Vinson et al. (2010) showed that signs and mouthings are processed largely in separate channels, our findings 
show that the two are tightly linked in terms of frequency and co-occurrence. Since there is no necessity for inhibition of mouthings in the production of manual signs, the mental representations of the two languages may be even more linked for bimodal bilinguals than for unimodal bilinguals.

We can only speculate as to why we did not find any differences in the use of code-blends in different groups of signers. The explicit attention to spoken Dutch in deaf education may have been such a dominant part of primary education for all signers that any variations in educational approach by different schools or at different times was only seen in the amount of (positive or negative) attention for NGT. An alternative possibility is that we looked at the wrong dependent variables, namely the frequency of mouthing. Traditional sociolinguistic and more recently sociophonetic studies of spoken (and signed) languages look at details of the pronunciation, which is something we did not do. We know from personal experience that deaf speakers adopt regional accents in their speech even if they only had visual access to spoken language all their life. Thus, one can clearly distinguish Flemish versus northern Dutch deaf speakers after hearing only a few syllables. While the attention for visible speech is increasing (e.g. Jesse \& Massaro, 2010; Van der Zande, 2013), we know of no studies looking at dialectal variation in visible oral articulations. Perhaps investigating the fine articulatory detail of mouthings could bring to light group differences that did not appear using the present methodology. At the same time, in so doing one might well be investigating speech characteristics that differ in corresponding groups in the hearing language community, while not establishing differences in relative role of spoken language in the sign language for these groups. By looking at frequency of spoken language elements in relation to manual elements in the way we did, we obtain a more direct measure of the relative importance of mouthings.

We conclude that spoken Dutch provides an almost continuous stream of linguistic information in parallel with the manual sign stream, and that the codeblends identified by Emmorey for bilingual hearing native signers of ASL are a core feature in the language of all deaf users of NGT. It remains an open question which of the two information channels is more important in activating lexical items during language perception. Deaf people are known to be proficient lip readers (Bernstein, Tucker, \& Demorest, 2000; Mohammed, Campbell, MacSweeney, Barry, \& Coleman, 2006), and given the prominence of (parts of) Dutch words in deaf interaction as demonstrated in our study it would appear plausible that they use lipreading as a strategy in communicating with other deaf signers. Posing this question may appear 
86 | Chapter 4: The prominence of spoken language elements in a sign language

to touch on the sensitive question of sign languages being full-fledged linguistic systems independent from spoken languages, but the present study suggests that there is no sign language without Dutch mouthings for deaf people in the Netherlands. In other words, there are no monolingual users of NGT, even if their level of proficiency in spoken Dutch may be variable. While this does not contradict the overwhelming evidence that NGT like other sign languages has a lexicon and a grammar that is markedly different from the related spoken language, it is unlikely that processing of sign language input is fully independent from processing spoken language, opening up a new domain of research in bilingualism studies. 


\section{Chapter 5: Bimodal code-mixing: speech supported signing is the norm for deaf NGT signers}

Slightly adapted from: Bank, R., Crasborn, O., \& Van Hout, $R$. (submitted). Bimodal code-mixing: speech supported signing is the norm for deaf NGT signers.

\subsection{Introduction}

W hen speakers use multiple languages in ordinary conversation they may mix those languages effortlessly in everyday use, so-called intra-sentential code-mixing. However, speakers also manage to keep the two languages separate in ordinary production, even though there is ample evidence that several languages are activated at once (e.g. Van Heuven \& Dijkstra, 2010). This apparent paradox raises the following question: is it harder to keep your languages separate or to mix them? In spoken languages, there is often a clear sanction on mixing: sometimes it simply is not appropriate from the perspective of audience design, when for instance the expectations of the addressee may be monolingual.

These sanctions may be absent when one of the languages is a signed language, since there is no external need to inhibit either of the articulatory channels (Emmorey, Petrich, \& Gollan, 2012; Morford, Wilkinson, Villwock, Piñar, \& Kroll, 2011). Thus bimodal code-mixing may throw an interesting light on the ease of mixing question. 
Deaf communities in western societies, having been subjected to oral education for several generations while at the same time using signed language as their primary and preferred language, are de facto bimodal bilingual. The lack of needing to inhibit spoken language results in the occurrence of those spoken language elements while signing, called 'mouthings' (see the contributions to Boyes Braem \& Sutton-Spence, 2001). These mouthings are generally viewed as having a one-on-one relationship with the manual sign they co-occur with. This relationship comprises two components, a temporal one and a semantic one. A typical mouthing is roughly timealigned with the manual sign and it also carries approximately the same meaning as the manual sign. Thus, it conveys mainly redundant information (e.g. for NGT see Bank, Crasborn, \& Van Hout, 2011). The grammar of NGT sentences leads to sign order patterns that may be very different from word order patterns in spoken Dutch. The manual signs comprise the primary information stream, with mouth gestures and mouthings accompanying the signs as a secondary information stream, a bimodal form of code-mixing, or 'code-blending' (Emmorey, Borinstein, \& Thompson, 2005; Emmorey, Borinstein, Thompson, \& Gollan, 2008).

For unimodal, sequential code-mixing in spoken languages, Muysken (2013), in an update of his (2000) account, distinguishes four types of underlying processes: insertion, alternation, congruent lexicalization, and backflagging. Given that manual information in sign language is the primary information stream, insertion seems to match code-blending best, as it is defined as the occurrence of lexical material from one language into the matrix or base language (Muysken, 2013: 714). Bank et al. (2011) suggested the term 'lexical addition' to substitute 'lexical insertion', since there is no real switch from one language to another. Van den Bogaerde and Baker (2005), in a longitudinal study on code-mixing between deaf mothers and their deaf or hearing children, found that the "type of code-mixing process that primarily occurred is congruent lexicalization with just some lexical insertion" (2005: 172). Muysken's description of code-mixing, however, does not take into account that signed languages have the intrinsic capability of simultaneously expressing different languages in different modalities: signing on the hands, and spoken language on the mouth. Cognitive limitations aside, there is in principle nothing to stop a signer from expressing two distinct grammars simultaneously. However, also in cases of codeblended signed and spoken language, one of the two languages will serve as the base language or matrix language (Muysken, 2000; Myers-Scotton, 2006), embedding material from the other language in its structure. Thus, in the case of NGT combined 
with mouthings (the default manifestation of NGT in everyday use, see Bank, Crasborn and Van Hout, under review), NGT can be considered the matrix language; the elements from spoken Dutch (i.e. mouthings) being the embedded language or guest language.

Another type of mixing between NGT and Dutch is found in 'sign-supported speech' (Nederlands met Gebaren, NmG). Although there is no formal system of manually coded Dutch such as has been described for Signing Exact English in North America (Gustason, Pfetzing, \& Zawolkow, 1975), the use of spoken Dutch with supporting signs is very common in everyday life. It is characterised as a contact variant for specific situations, where the primary audience is hearing but manual lexical items from the sign language are mixed in to ensure that the deaf audience gets more perceptual input for the recognition of the spoken language sentences. Terpstra and Schermer (2006) describe NmG as a continuum between NGT and Dutch where three main forms can be distinguished. In each of these cases, Dutch grammar forms the basis, and can be combined with elements of NGT to various degrees. At the Dutch end of the continuum, spoken content words are combined with signs, while at the NGT end of the sign-supported speech continuum, both vocabulary and grammatical elements from NGT are combined with Dutch sentences, occasionally leading to grammatically incorrect Dutch sentences. The hypothesis we explore in this paper is that in communicating with each other, native and near-native signers mix Dutch and NGT in yet another way: NGT grammar forms the starting point, and Dutch is mixed in to various degrees. In a sense, we expect to find the mirror image of what Terpstra and Schermer have described for NmG, leading to a form of language contact that would merit a new abbreviation 'GmN', Gebaren met Nederlands or 'speechsupported sign'.

As indicated above, mouthings are usually roughly time-aligned with the manual sign and they are semantically congruent. However, there are exceptions to both the temporal alignment and semantic congruency. One such exception, in temporal alignment, is the spreading of mouthings over adjacent signs, so that one mouthing co-occurs with multiple signs. Bank, Crasborn, and Van Hout (2013) found this to be a frequent phenomenon in NGT, confirming the initial observations of Crasborn, Van der Kooij, Waters, Woll, and Mesch (2008) that most mouthings spread rightward (progressively) and encompass one adjacent sign. However, leftward (regressive) spreading occurs as well, and the scope of spreading was found to extend over up to five signs. 
Another such exception, in semantic overlap, is the variation in the Dutch lexical items that may accompany a manual sign (Schermer, 1990). Bank et al. (2011), looking at the highly frequent signs in the Corpus NGT, showed that almost all mouthings - both in reduced and in fully pronounced forms - either denote the same meaning as the manual signs they accompany, or denote something very close to that (like GOOD with prima ('excellent'), for instance). For signs that are fairly consistently accompanied by the same mouthing, the mouthing may form part of the lexical representation of the sign (Bank et al., 2011).

The current paper focuses on mouthings in NGT and aims to establish what happens when there is no neat semantic overlap between manual sign and mouthing (as above), or when Dutch lexical items are fit into the manual signing stream without any manual counterpart. An example of the latter is given in (5.1). To give an idea of the temporal alignment of the left and right hand glosses and mouth annotations, we will add screenshots of the annotations to the examples. Details on how to read the screenshots will be given at the end of the methodology section, below.

$\begin{array}{llll}\text { ID-gloss: } & \text { GROW-UP } & \text { ORAL } & \text { MORE ORAL } \\ \text { Mouth: } & \text { ouwer } & \text { ouwer } & \text { meer met mond }\end{array}$

Mouth gloss: old.COMP older.COMP more with mouth more lipread.INF Utterance translation: 'The older they are, the better they are at speechreading'

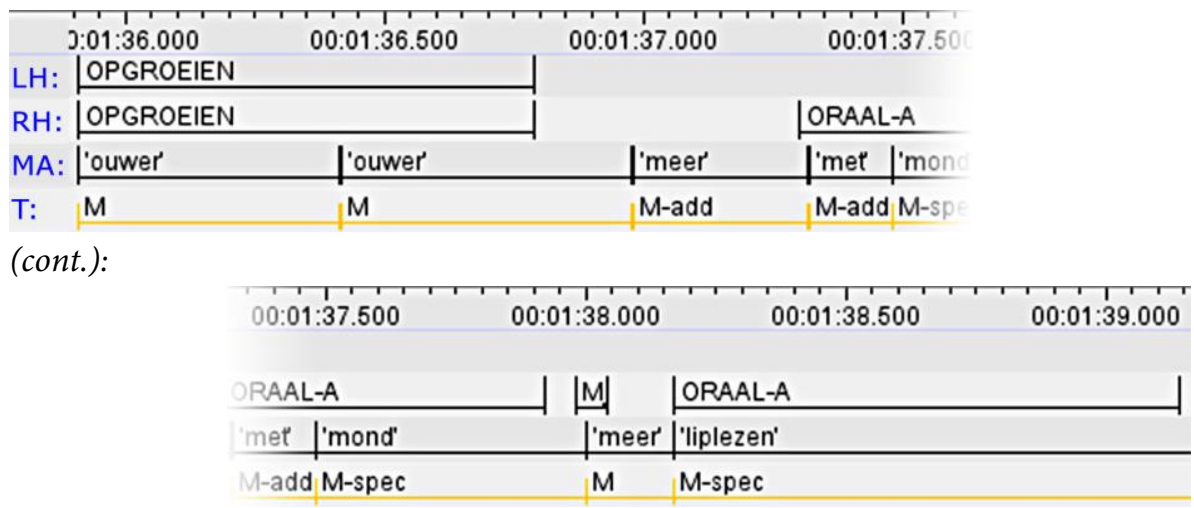

In (5.1), there are two extra mouthings added to an NGT-sentence, one just before and the other co-occurring with the onset of the first occurrence of the sign ORAAL ('ORAL'). The mouthing meer ('more') occurs during the transitional movement that 
the hands make between the sign OPGROEIEN ('GROW-UP') and 'ORAL', and the mouthing met ('with') is made while the hands have already begun the articulation of 'ORAL', that later is accompanied by its own mouthing, mond ('mouth'). 'The thing to note here is that during the transitional movement between the two signs, linguistic material on the mouth is being inserted into the information stream.

It would be hard to classify this construction as NmG, since we don't see a Dutch sentence here. On the other hand, the use of met ('with') is not common in NGT and is influenced by how Dutch prepositional phrases are constructed, although the phrase met mond 'with mouth' in Dutch would require an article (met de mond 'with the mouth') to be well-formed in spoken Dutch. This example raises the question whether all sentences with added or specifying mouthings are similar in nature, being a mix of NGT and Dutch that is more speech-supported sign than sign-supported speech.

The occurrence of these insertions of added mouthings suggests that the lexicons of NGT and Dutch may be combined in more complex ways than by directly linking individual signs and words, calling for a sentence-level analysis. We will explore to what extent and in which way NGT and Dutch can be combined to convey complex messages.

We will use the same corpus as the studies above (Bank et al., 2011, 2013, under review) and categorise the various occurrences of added mouthings and semantic mismatches. In addition to standard mouthings, we will distinguish three varieties of special mouthings in this paper:

1) Solo mouthings are isolated words or short phrases that occur while the hands are in rest position. Solo mouthings are commonplace in the Corpus NGT (Nedela, 2013). They are most often used as a backchannel, a short feedback cue where a signer mouths a short ja ('yes') or okee ('okay') to signal the interlocutor that he or she is following the flow of the conversation but does not claim the turn. Since we are interested in mouthings on the sentential level and how they relate to signing, we will only briefly take these backchannels into account in the current study, focussing for the most part on longer strings of

\footnotetext{
${ }^{9}$ It could be argued that the actual accompanying mouthing for the first occurrence of ORAL is met mond ('with mouth') instead of just mond, but in that case we still would have meer as an added mouthing.
} 
solo mouthings, and solo mouthings embedded in a sentence when the hands may temporarily move to a rest position.

2) Added mouthings occur in the stream of mouth actions that accompanies the manual signing stream, but an added mouthing does not accompany any specific sign; rather, they are squeezed in between two mouthings, occurring during transitional movements of the hands, or overlapping a manual sign that comes with its own mouth action. There is a continuum between two extremes on which an added mouthing can occur. On one end of that continuum, a mouthing occurs between two signs that each have their own mouth action, and the added mouthing occurs during the transitional movement between the signs, not overlapping either sign. On the other end, a mouthing completely overlaps with a sign that also is accompanied by its own mouth action, so that there are two mouth actions articulated during one sign.

3) Specifying mouthings are (roughly) time-aligned with a manual sign but do not denote the same semantic concept. Rather, they specify the meaning of the manual sign or they add a distinctive meaning to it.

For each of the three categories, we aim to establish what their semantic and/or morphosyntactic contribution is at the sentence level.

\subsection{Methodology}

We analysed parts of the Corpus NGT (Crasborn \& Zwitserlood, 2008b; Crasborn, Zwitserlood, \& Ros, 2008). This corpus contains video data of 92 prelingually deaf signers, recorded in pairs, who retell video clips and picture stories, and discuss issues related to deafness, education and sign language. The great majority of signers have been educated at minimally secondary school level, with Dutch as the primary language of instruction and NGT playing a secondary role at best. NGT was for none of the signers a subject language in school, but started to make its way into education for the younger signers as a language of instruction. In most cases, the language of instruction was spoken Dutch (for the older generations) and spoken Dutch alternated with sign-supported speech for children educated from 1980 onwards (people younger than 30 in our data set). The Corpus NGT is the best effort to date to record a representative sample of NGT use of the core of the deaf community, consisting of born-deaf or early-deafened signers, most of whom have not grown up 
in deaf families. All signers were recorded in dyads where people were matched in generation and regional background; the members of each dyad knew each other well and frequently interacted with each other in daily life.

Annotation of the corpus is ongoing; currently almost 137,000 glosses have been added for the hands, and over 13,000 for the mouth. ${ }^{10}$ At present, the corpus contains almost 320,000 annotations for different levels of transcription and analysis, from sentence translations to degree of thumb extension. For the current paper, we used a subset of 40 video clips from the corpus that were fully annotated for hands and mouth, spanning 94 minutes of signed conversation from 40 signers. All mouth annotations include a child annotation containing the type of mouth action: ' $\mathrm{M}$ ' for mouthings (further specified with 'M-add' for an added mouthing, ' $M$-spec' for a specifying mouthing, or 'M-solo' for a solo mouthing); ' $\mathrm{A}$ ' for adjectival/ adverbial mouth gestures, specifying the sign; ' $W$ ' for whole face movement, a global facial expression; '4' for mouth-for-mouth, where the mouth performs the action (like in KISS or CHEW); 'E' for semantically empty mouth gestures (see Crasborn, Van der Kooij, Waters, Woll, \& Mesch, 2008, for a more detailed description). We went back to our annotations to check whether all solo mouthings, added mouthings and specifying mouthings were correctly annotated, including their alignment with the manual glosses, and created new sentence-level annotations for sentences containing added or specifying mouthings. This resulted in 266 new annotations containing both the manual glosses and orthographic mouth transcriptions, allowing us to have a quick overview of all relevant sentences. Further, we asked two deaf native signers who are fluent in Dutch to translate these sentences, with the instruction to not only focus on the meaning of the manual signing, but to take all visible head and body movement into account. By looking at the semantics of the whole utterance, we were able to establish what the additional value is of mouthings in utterances. The information on the gloss, mouth, and translation tiers formed the basis for our analyses.

The examples that we will discuss below will contain screenshots of our annotations, to give an idea of the temporal alignment of the left- and right-hand glosses and the mouth annotations. In all screenshots, the top row is a timeline

\footnotetext{
${ }^{10}$ We use the ELAN annotation software (Wittenburg, Brugman, Russel, Klassmann, \& Sloetjes,
} 2006) for our annotation work, available at http://tla.mpi.nl/tools/tla-tools/elan/. 
indicating the length of the annotations and the position in the file, in the format hours:minutes:seconds.milliseconds. Below the timeline are four tiers: a tier with ID glosses (see below) for the Left Hand (LH; may be empty in case of only onehanded signs by a right-handed signer), a tier with ID-glosses for the Right Hand (RH), a tier for Mouth Annotations (MA) and a tier indicating the Type of mouth action ( $\mathrm{T})$. Any whitespace between sign glosses reflects a transitional movement between two signs. In the text of the examples, we collapse the two ID-gloss tiers into one line, as differences in handedness or the use of one- vs. two-handed signs are not relevant to the present study. We will give the English equivalent of the Dutch IDglosses used in annotating the corpus. It should be noted that ID-glosses are just labels; however, we chose the glosses so that, in general, the Dutch word (and the English translation) used for that gloss is semantically corresponding to the sign it represents. The glosses PT and PT:1 refer to a pointing sign in a general direction and a pointing sign to self, respectively. Sometimes, the screenshots show ID-glosses that end with an affix like -A or -B; this indicates the variety of the sign that is used and is not relevant for our discussion. Fingerspelling is indicated with a hash sign (\#), false starts are indicated with a tilde $(\sim)$.

The mouth annotations in the screenshots, the Dutch orthographic representations of the visible speech elements, are repeated in the text of the examples; mouth gestures are indicated by their type, 'A', 'E', '4', or 'W'. Although providing screenshots of the gloss alignments may be helpful and concise, they do not contain all relevant linguistic events present in the interaction (leaving out non-manual cues, for instance). The reader is therefore encouraged to follow the links provided in the appendix that point to the relevant sections of the online version of the Corpus NGT.

\subsection{Results}

There are an estimated 2,066 sentences in our 40-signer, 94-minute sample. ${ }^{11}$ This includes 266 utterances with a non-standard (i.e. added, specifying or solo) mouthing

\footnotetext{
${ }^{11}$ Because annotation work is very time consuming, we only have 57 minutes of clips that are fully annotated on the sentence level, $61 \%$ of our sample. These clips contain 1,254 utterances, including 161 utterances with a non-standard mouthing. Extrapolating to $100 \%$ (94 minutes)
} 
occurring in that utterance, sometimes with multiple occurrences per utterance. These 266 utterances, about $12 \%$ of the total of 2066 , are expressed by 36 signers and contain 359 added mouthings, 39 specifying mouthings and 38 solo mouthings.

We will first show examples of standard mouthings (section 5.3.1) and solo mouthings used in backchanneling (5.3.2). We then turn to multiple solo mouthings used in short strings (5.3.3), mouthings specifying semantic information in content words (5.3.4), the use of Dutch function words in added mouthings (5.3.5), and Dutch word order and Dutch idiomatic expressions (5.3.6).

\subsubsection{Standard mouthings}

As stated in the introduction of this paper, there is usually a semantic and temporal relationship between a mouthing and the manual sign it co-occurs with. This is neatly illustrated in (5.2), in which most signs are accompanied by a mouth action, mostly mouthings, and these mouthings in turn are time-aligned with and have the same meaning as the signs they accompany. Following Bank et al. (2011), we call these mouthings 'standard mouthings'.

\section{ID-gloss:}

Mouth:

Mouth gloss:

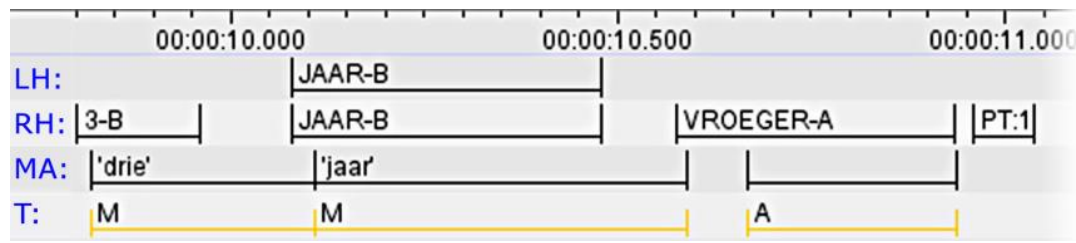

(cont.):

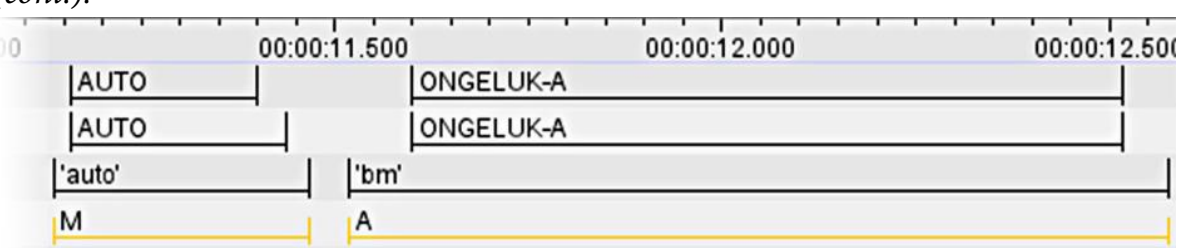

results in 2,066 utterances, including 266 with non-standard mouthings, which is exactly the number of annotations we indeed have made for this purpose. 
96 | Chapter 5: Bimodal code-mixing: speech supported signing is the norm for deaf NGT signers

The three mouthings in this phrase (indicated by $\mathrm{M}$ in the lowest tier in the screenshot) are all standard mouthings: the sign glossed as 3 is aligned with the mouthing drie ('three'), JAAR ('YEAR') is aligned with jaar ('year'), and AUTO ('CAR') is aligned with auto ('car'). There is no mouthing accompanying PT:1 (the pointing-toself between VROEGER ('PAST') and 'CAR'), and there are adjectival mouth gestures together with the signs glossed as 'PAST' and ONGELUK ('ACCIDENT').

\subsubsection{Solo mouthings used in backchanneling}

There are 211 annotations labelled as solo mouthing in our sample of 94 minutes, including the 38 that are contained in longer strings of mouth actions (to be discussed in section 5.3.3). The remaining 173 solo mouthings occur isolated from any other mouth actions or manual signs. In most cases the solo mouthings are used as backchannels, the short feedback cues from the interlocutor. Most often this is a simple ja ('yes', 32\%) or nee ('no', 12\%), to signal the interlocutor that the signer is paying attention. About $6 \%$ of the tokens were unintelligible, and the remaining $50 \%$ of isolated solo mouthings consists of single tokens of all kinds of words, often merely repeating what the interlocutor just signed. A few examples of these tokens are december ('December'), nodig ('necessary') and woon ('live-in'), to name just a few. This is quite like the backchanneling behaviour in spoken languages (Duncan, 1974; McCarthy, 2002; Wong \& Peters, 2007), and also not unlike manual backchanneling in sign languages (Mesch, Nilsson \& Wallin, 2011). We did no in-depth analysis of solo mouthings used in backchanneling, we focussed instead on solo mouthings occurring in the mouth action stream, to be discussed next. 


\subsubsection{Solo mouthings used in short strings}

The 38 solo mouthings are contained in 18 utterances. There are a few utterances made up from a string of solo mouthings only, for example in (5.3), where there is no manual activity at all.

(5.3) ID-gloss:

Mouth: ja klopt mooi weer

Mouth gloss: yes right nice weather

Translation: 'Yes, that's right, the weather was good'

\begin{tabular}{|c|c|c|}
\hline \multirow{2}{*}{\multicolumn{3}{|c|}{ LH: }} \\
\hline & & \\
\hline \multicolumn{3}{|l|}{$\mathrm{RH}$ : } \\
\hline MA:|'ja' & |'klopt & |'mooi' \\
\hline T: $\quad$ M-solo & M-solo & M-solo \\
\hline
\end{tabular}

The signer agrees here with what the other signer says, not as a backchannel but confirming that indeed that the weather was good in the period under discussion. One of the reasons why the signer decides to use mouthings only and no signs may be that she has her hands folded around her knee. There a few more cases in our sample where a signer's hands are not directly available for singing because of folded arms or hands. Sometimes, however, signers do have their hands available, but still choose to use only mouthings for short sentences. Occasionally, utterances start out with mouthings only, and signing starts only halfway the sentence. Example (5.4) illustrates this, where also the end of the utterance is only mouthed. Both at the start and at the end of the utterance, the signer has her hands in rest position, in her lap. 
98 | Chapter 5: Bimodal code-mixing: speech supported signing is the norm for deaf NGT signers

(5.4) ID-gloss:

Mouth:

maakt niet uit niet nodig alleen slechtho-

Mouth gloss:

make.3SG not out not necessary only hard_of-

(cont.):

ID-gloss:

EAR

NECESSARY SIGN

Mouth:

slechthorenden hoeft niet gebaren hoeft niet

Mouth gloss: hard_of_hearing need.3SG not sign.INF need.3SG not

Utterance translation: 'No that doesn't matter, it is not needed because the hard of hearing can talk and hear, signing is not necessary in that case'

\begin{tabular}{|c|c|c|c|c|c|}
\hline \multicolumn{4}{|c|}{$00: 03: 10.500$} & $00: 03: 11.000$ & $00: 03: 11.500$ \\
\hline \multicolumn{6}{|l|}{ LH: } \\
\hline \multicolumn{6}{|l|}{ RH: } \\
\hline MA: & |'maakt' & 'niet' & 'uit' & 'niet' & 'nodig' \\
\hline $\mathrm{T}:$ & M-solo & M-solc & $\mathrm{M}-\mathrm{sc}$ & M-sol & M-solo \\
\hline
\end{tabular}

(cont.):

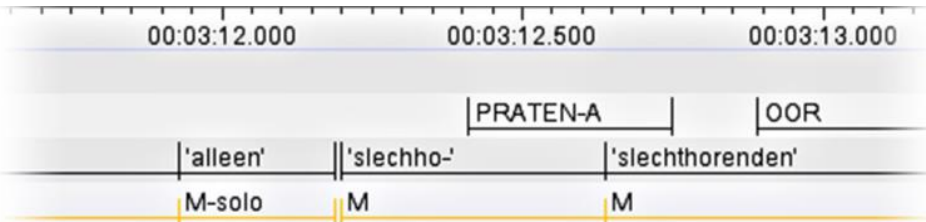

(cont. 2):

\begin{tabular}{|c|c|c|c|c|}
\hline \multicolumn{2}{|c|}{ 00:03:13.500 } & $00: 03: 14.000$ & & $00: 03: 14.500$ \\
\hline & GEBAREN-A & & & \\
\hline $\sim \mathrm{NO}$ & GEBAREN-A & & & \\
\hline 'hoeft^niet' & |'gebaren' & & |'hoeft & |'niet' \\
\hline$M$ & $M$ & & M-solo & M-solo \\
\hline
\end{tabular}

In the case of isolated strings of solo mouthings, the matrix language is clearly spoken Dutch (such as in 5.3) In (5.4), there is alternation between NGT and spoken Dutch. But mostly, the matrix language remains NGT, with a few mouthed words before or after a signed sentence. Occasionally, a solo mouthing occurs in the middle of a (compound) sentence, such as the conjunction in (5.5). The signer brings her hand to her lap, mouths maar ('but'), and then continues signing. 
$(5.5)$

ID-gloss: PT:1 FEEL DEAFCHILD PT:1 HEAR APPROX PRESENT Mouth: ik graag doof kind maarhorend ook bij Mouth gloss: I gladly deaf child but hearing also present Utterance translation: 'I would like to have a deaf child, but a hearing child would be welcome too'

\begin{tabular}{|c|c|c|c|c|c|c|}
\hline & $00: 04: 22.000$ & $00: 04: 22.500$ & $00: 04$ & 23.000 & 00 & \\
\hline \multicolumn{7}{|l|}{ LH: } \\
\hline $\mathrm{RH}:$ & PTI VOELEN-| & DOOF-A & KIND-B & PT:1 & & \\
\hline MA: 'ik & ||'graag' & |doof & 'kind' & & t & \\
\hline $\mathrm{T}: \mathrm{M}$ & M-spec & M & $M$ & & & \\
\hline \multicolumn{7}{|l|}{ (cont.): } \\
\hline & \multirow[t]{2}{*}{423.500} & $04: 24.000$ & \multicolumn{2}{|l|}{$00: 04: 24.500$} & $00: 04: 25.000$ & $00: C$ \\
\hline & & & HOREN-A| & \multicolumn{2}{|c|}{ ONGE) ERBIJ-A } & \\
\hline & 'maar' & 'horend' & & 'ook 'b & ij' & \\
\hline & M-solo & $M$ & & $M$ & & \\
\hline
\end{tabular}

There are fourteen different signers in our sample who make at least one solo mouthing. Solo mouthings thus clearly form a part of communication, and they give a sense of Dutchness to the message.

\subsubsection{Specifying semantic information in content words (specifying mouthings)}

There are 39 specifying mouthings in our sample, contained in 31 utterances. In (5.6), there is a mouthing that specifies the sign CONTACT ('CONTACT'), a two-handed sign that is made twice here (the second gloss of 'CONTACT' on the left hand is still part of the first occurrence of 'CONTACT'). During the first occurrence, there is initially no mouth activity; then, the left hand briefly interrupts signing 'CONTACT' in order to sign MAKKELIJK ('EASY') - aligned with a (reduced) standard mouthing makkelijk ('easy') spreading over the last part of 'CONTACT'. The second occurrence of 'CONTACT' has an accompanying mouthing, but it is not the standard mouthing contact. Instead, there is the specifying mouthing moeilijk ('difficult'), adding meaning to the sign. The repetition of the sign here, with a different mouthing than accompanying the first occurrence, makes it clear that the signer is contrasting two types of contact, avoiding the need to sign 'DIFFICULT' as well. 
(5.6)

\section{ID-gloss:}

Mouth:

Mouth gloss:

Utterance translation: 'Contact [with deaf children] is easy, but contact [with hearing children] is difficult.'

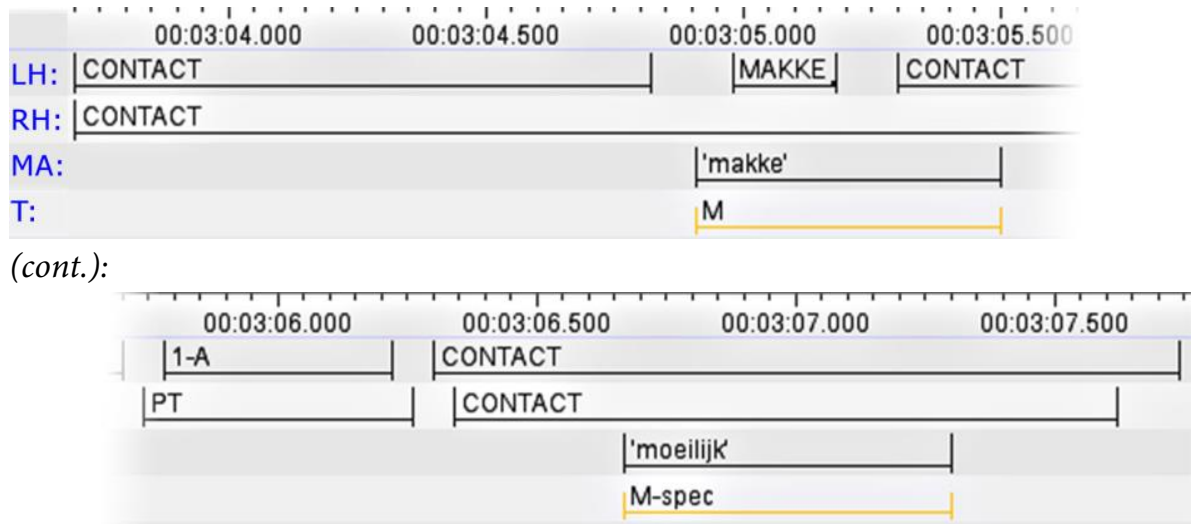

Example (5.7) shows a more condensed form of contrasting. The sign glossed as REGIO ('REGION') is a one-handed sign that is articulated two-handed here, thus contrasting two different locations in signing space, one with the right hand and one with the left hand. The mouthing verschil ('difference') further intensifies the contrasting of locations by the two hands. As explained in section 5.2 above, the $W$ on the mouth type tier stands for a mouth gesture where the whole face is involved.

$\begin{array}{llll}\text { ID-gloss: } & \text { REGION } & \text { EVEN-SO } & \text { PALM-UP PT } \\ \text { Mouth: } & \text { verschil } & W & \text { toch } \\ \text { Mouth gloss: } & \text { difference } & \mathrm{W} & \text { even_so }\end{array}$

Utterance translation: 'There are regional differences after all.'

\begin{tabular}{|c|c|c|c|c|}
\hline .00 & $00: 02: 25.500$ & $00: 02: 26.000$ & & $00: 02: 26.5$ \\
\hline LH: & |REGIO-B & $\mathrm{TOCH}$ & $\mathrm{PO} \mid$ & \\
\hline RH: & & $\mathrm{TOCH}$ & $\mathrm{PO}$ & PT \\
\hline \multicolumn{2}{|c|}{ MA: |'verschil' } & \multicolumn{3}{|c|}{ closed, st 'toch' } \\
\hline $\mathrm{T}:$ & & W? & & \\
\hline
\end{tabular}


In (5.8), there is nothing to contrast, but a sign is modified by its mouthing to indicate something is re-occurring. The sign glossed as DISCUSSIEREN ('DISPUTE') is combined with the mouthing altijd ('always'), to mean 'always having an argument'. Like in the previous examples, there is a lexical sign (in this case 'ALWAYS') for the specifying mouthing that is used here, but the signer rather chooses to combine a sign and a modifying mouthing instead of two signs.

(5.8) ID-gloss: Mouth: Mouth gloss:
SEE DISPUTE EVERY DISPUTE gezien altijd altijd see.PRF always always

Utterance translation: 'They saw we always had an argument, every day.'

\begin{tabular}{|c|c|c|c|}
\hline & .500 & $00: 05: 09.500$ & $00: 05: 10.000$ \\
\hline LH: & & \multicolumn{2}{|l|}{ DISCUSSIEREN } \\
\hline $\mathrm{RH}$ : & ZIEN-A & \multicolumn{2}{|l|}{ DISCUSSIEREN } \\
\hline MA: & 'gezien' & 'altijd' & |'altijd' \\
\hline T: & $M$ & M-spec & M-spec \\
\hline
\end{tabular}

(cont.):

|ELK)

Interestingly, the sign ZIEN ('SEE') is aligned with the mouthing gezien ('have_seen'); this is an instance of temporal inflection of a mouthed verb. Although there is no system for temporal inflection in NGT, use of perfect participles in mouthings is not common in our data set.

\subsubsection{Use of Dutch words in added mouthings}

As stated above, added mouthings are defined as mouthings that do not accompany a specific sign, but are squeezed in between two signs, or occur during transitional movements. They differ from solo mouthings in that the hands are not in rest position during the added mouthing, but active articulating another sign or in transition between two signs. We found 359 added mouthings in our set of 266 utterances. All 
word classes are present, but they vary a lot in how many different tokens are present per word type in that class, as is summarised in Table 5.1.

Table 5.1. Word classes occurring as added mouthings, their token and type counts and ratios.

\begin{tabular}{llrrlllc}
\hline \hline Word class & $\begin{array}{l}\text { Token } \\
\text { count }\end{array}$ & $\begin{array}{l}\text { Type } \\
\text { count }\end{array}$ & $\begin{array}{l}\text { Tokens/ } \\
\text { Type }\end{array}$ & Word class & $\begin{array}{l}\text { Token } \\
\text { count }\end{array}$ & $\begin{array}{l}\text { Type } \\
\text { count }\end{array}$ & $\begin{array}{l}\text { Tokens/ } \\
\text { Type }\end{array}$ \\
\hline Conjunction & 70 & 5 & 14.0 & Wh-question & 25 & 4 & 6.3 \\
Verb & 70 & 18 & 3.9 & Noun & 16 & 11 & 1.5 \\
$\quad$ Copular verb & 30 & 2 & 15.0 & Pronoun & 16 & 9 & 1.8 \\
$\quad$ Lexical verb & 22 & 10 & 2.2 & Adjective & 6 & 6 & 1.0 \\
$\quad$ Modal verb & 12 & 4 & 3.0 & Interjection & 4 & 2 & 2.0 \\
$\quad$ Aux. verb & 6 & 2 & 3.0 & Article & 3 & 3 & 1.0 \\
Adverb & 61 & 25 & 2.4 & Numeral & 3 & 2 & 1.5 \\
Preposition & 52 & 12 & 4.3 & Demonstrative & 1 & 1 & 1.0 \\
Negation & 32 & 2 & 16.0 & & & & \\
\hline \hline
\end{tabular}

The three words that are most frequently used as added mouthings are the conjunction maar ('but', 43 tokens), the copular verb zijn ('to be', 30 tokens) and the negation niet ('not', 26 tokens). We will discuss some examples from the five groups with the largest token/type ratio: conjunctions, prepositions, negations, copular verbs and wh-questions.

\subsubsection{Conjunctions}

The 70 conjunctions in our collection of added mouthings are predominantly coordinating ones. We counted 43 tokens of maar ('but'), 20 tokens of of ('or'), 4 times als ('if), twice en ('and'), and one token of omdat ('because'). Example (5.9) illustrates how a signer deploys two strategies for contrasting DEAF versus HEARING. One is dominance reversal (DEAF and the preceding signs are signed with the left hand, HEARING and the following signs are signed with the right hand), the other is the addition of the mouthing maar ('but'). 
(5.9)

$\begin{array}{lll}\text { ID-gloss: } & \text { PT:1 SELF DEAF } & \text { HEAR NOT } \\ \text { Mouth: } & \text { zelf doofmaar horend niet } \\ \text { Mouth gloss: } & \text { self deaf but hear.PTCP not }\end{array}$

Utterance translation: '[I accept that] I am deaf myself, but hearing people don't.'

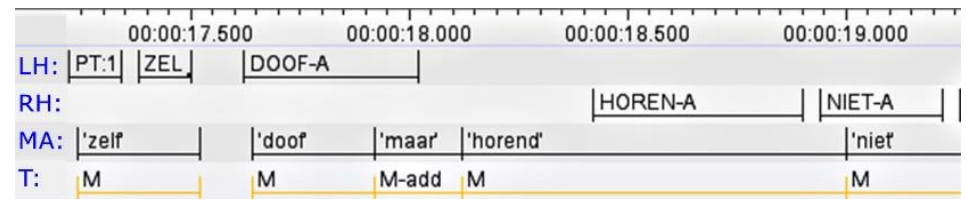

Another example of the use of a conjunction is given in (5.10). Here, all signs (except for the final palm-up) are made with only the right hand, and the signer wants to contrast being deaf with not being deaf. This contrast is made non-manually by first nodding and then shaking the head, and also by a combination of the added mouthing of ('or') and the sign NIET ('NOT'). Note that a manual negation is present here, which is not necessarily always the case (see section 5.3.5.3, below). For the duration of the annotation 'not visible', the signer had his hand between the camera and his face; it is likely that he pronounced the full verb ontdekken ('discover') or a reduced version of it.

(5.10) ID-gloss: Mouth: Mouth gloss:

Utterance translation: 'We want to find out for ourselves whether it is deaf or not'

\begin{tabular}{|c|c|c|c|c|c|}
\hline 00:01: & & 00:01:17.00 & & $01: 17.500$ & $00: 01: 18.000$ \\
\hline \multicolumn{6}{|l|}{ LH: } \\
\hline RH: |PT:1 & ZELF & ZIE & KIJKEN-A & ONTDEKKEN & \multirow{2}{*}{$\begin{array}{l}\text { DOOF-B } \\
\text { |'doof }\end{array}$} \\
\hline MA: & |'zelf' & & |'on & not visible & \\
\hline T: & M & & M & not visible & $M$ \\
\hline
\end{tabular}

(cont.):

00:01:18.500


104 | Chapter 5: Bimodal code-mixing: speech supported signing is the norm for deaf NGT signers

\subsubsection{Prepositions}

There are 52 added mouthings that classify as prepositions, in order of decreasing occurrence: voor ('in front of), om ('around' or 'in order to'), op ('on'), in ('in'), met ('with'), bij ('near'), van ('of'), te ('to'), door ('through'), naar ('to'), vanaf ('from'), and volgens ('according to'). In NGT, most spatial prepositions can be expressed lexically. Other spatial relations can be expressed using classifier constructions, or positioning objects in signing space. There are no lexical signs for non-spatial prepositions. In example (5.11), om means 'in order to'. A correct Dutch sentence, however, would include the adverb te to make om te leren ('in order to learn'). Also, a correct Dutch sentence would have a different word order and not repeat the wh-question, indicating that NGT is the matrix language here.

(5.11) ID-gloss:

$$
\text { WHY LEARN BETTER TALK }
$$

Mouth: $\quad$ waarom waarom om leren beter praten

Mouth gloss: why why to learn.INF better talk.INF Utterance translation: 'Why? To learn to speak better.'

\begin{tabular}{|c|c|c|c|c|c|c|}
\hline & $01: 00.500$ & 00 & $1: 01.000$ & 00:01:01.500 & 00:01:02. & \\
\hline LH: & WAARC & & & & & \\
\hline $\mathrm{RH}:$ & WAARC & & & LEREN & & \\
\hline MA: & |'waarom' & 'waarom' & 'om' & |'Ieren' & 'beti & \\
\hline $\mathrm{T}:$ & M & $M$ & M-add & $M$ & M & \\
\hline (cont & & & & & & \\
\hline & & & $00: 01: 02.00$ & $00: 01: 02.500$ & 00:01:03.000 & $00: 01$ \\
\hline & & & & TER & PRATEN-A & \\
\hline & & & 'beter' & & 'praten' & \\
\hline & & & $M$ & & $M$ & \\
\hline
\end{tabular}




\subsubsection{Negation}

There are 32 cases of mouthed negation without a manual negation being present: 26 tokens of niet ('not'), and 6 tokens of nee ('no'). In all cases except one, non-manual negation was also present in the form of shaking the head. In (5.12), the mouthed negation is combined with a shaking of the head and a general purpose PALM-UP sign that has no intrinsic meaning of negation (recall that the hash sign (\#) denotes fingerspelling).

(5.12) ID-gloss:

PAST PALM-UP \#NGT NOT-YET PALM-UP

Mouth:

vroegerniet NGT nog niet

Mouth gloss:

past not NGT yet not

Utterance translation: 'We didn't have NGT back then, it wasn't there yet'

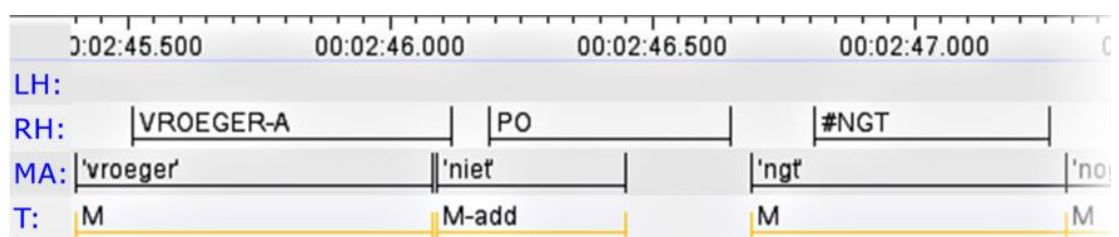

(cont.):

\begin{tabular}{|c|c|c|c|}
\hline $00: 02: 47.500$ & $00: 02: 48.000$ & $00: 02: 48.500$ & $00: 02: 49.000$ \\
\hline NOG-NIET & $\mid$ PO & & \\
\hline 'nog niet' & & & \\
\hline M & & & \\
\hline
\end{tabular}




\subsubsection{Copular verbs}

There are 31 copular verbs in our collection of added mouthings: 30 instances of zijn ('to be'), and one instance of worden ('to become'). There is no equivalent of a copular verb in NGT. In (5.13), the mouthing semantically associated with PT:1 is $i k$ ('I'), although that is articulated before the sign in both cases, so that the signs align with the copular verb ben ('am', first person singular of 'to be'). It can be argued that the signer associates the mouthed word pair $i k$ ben (' $\mathrm{I}$ am') with the sign PT:1, but the appearance of the copular verb here strongly suggests Dutch influence. However, the signer does not use a conjunction to connect the two parts of the sentence, and she uses an NGT word order in the second part (Dutch word order would be $i k$ ben 17 jaar oud 'I am 17 years old'). This indicates that NGT is the matrix language here.

(5.13) ID-gloss:

PT:1 DEAF PT:1 OLD 17 YEAR

Mouth: ikben doof ik ben oud zeventien jaar

Mouth gloss: I be.1SG deaf I be.1SG old seventeen year

Utterance translation: 'I am deaf and I'm seventeen years old.'

\begin{tabular}{|c|c|c|c|c|c|c|}
\hline \multicolumn{3}{|c|}{$00: 00: 59.500$} & $00: 01: 00.000$ & \multicolumn{2}{|c|}{$00: 01: 00.500$} & 00 \\
\hline \multicolumn{7}{|l|}{ LH: } \\
\hline $\mathrm{RH}:$ & PT & & DOOF-B & & PT & \\
\hline MA: 'ik & 'b & \multicolumn{2}{|c|}{ 'doof } & 'ik' & 'bel 'oud' & \\
\hline $\mathrm{T}: \quad \mathrm{M}$ & M. & \multicolumn{2}{|l|}{$M$} & M & $M-M$ & \\
\hline
\end{tabular}

(cont.):

\begin{tabular}{|c|c|c|c|}
\hline \multicolumn{2}{|c|}{ 00:01:01.000 } & $00: 01: 01.500$ & 00:01:0 \\
\hline & \multicolumn{3}{|l|}{ 17-C } \\
\hline & $17-C$ & \multicolumn{2}{|c|}{ JAAR-A } \\
\hline \multicolumn{2}{|c|}{ |'zeventien' } & |'jaar' & \\
\hline \multicolumn{2}{|c|}{ M } & M & \\
\hline
\end{tabular}

Just as with gezien ('have seen') in (5.8), there are occurrences of perfect participles in our sample, but these are rare cases. There is one instance of geweest ('have been') as an added mouthing, and one instance of geworden ('have become'). All other instances are either ben(t) ('am/are', first or second person singular, like in example (5.13); 17 cases) or is ('is', third person singular, nine cases). 


\subsubsection{Wh-question words}

Among the 25 wh-question words are 16 instances of hoe ('how'), 5 instances of wat ('what'), 3 instances of waar ('where') and one instance of waarom ('why'). All these words have signed equivalences in NGT. Example (5.14) shows a (short) occurrence of 'how', articulated while the hands are moving towards the start position of ouD ('OLD').

(5.14) ID-gloss: Mouth:

Mouth gloss:

Utterance translation: 'How old is your son now, eight?'

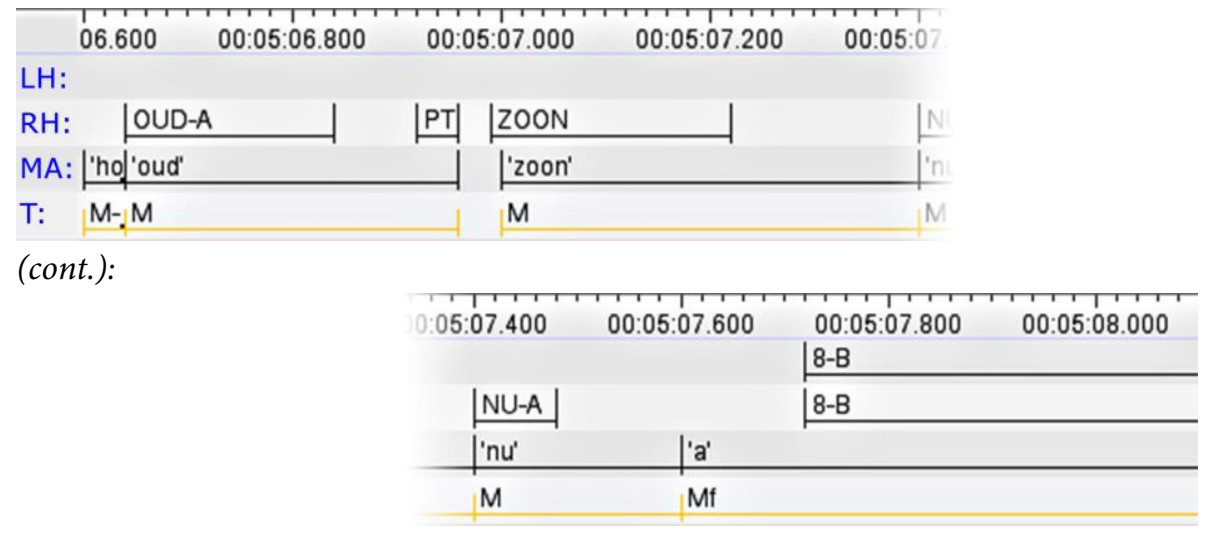

\subsubsection{Dutch word order and Dutch idiomatic expressions}

The examples shown so far, presenting solo, specifying and added mouthings, describe the multiple techniques that signers have available to blend some Dutch into their signing. We wanted to know to which extent this results in producing grammatically correct Dutch sentences together with signing. We found 18 such wellformed sentences on a total of 2,066, so it is the exception rather than the rule. Please note that the sentences we found are a by-product from our analysis of non-standard mouthings; there may be a few more in our 94-minute sample, if these mouthed sentences have the same grammatical structure as the NGT sentence they occur with. Given the observations on differences in grammar between Dutch and NGT (Bos, 
1990, 1993, 1995; Crasborn, Van der Kooij, Ros, \& De Hoop, 2009), we predict that there will not be many of such cases over 'accidental' overlap.

In (5.15), a copular verb and an adverb are added mouthings, and the sequence makes a well-formed Dutch sentence.

$\begin{array}{lll}\text { ID-gloss: } & \text { PT: } 1 \text { SISTER PT } & \text { DEAF } \\ \text { Mouth: } & \text { ja mijn zuster is ook doof } \\ \text { Mouth gloss: } & \text { yes I.POSS sister be.3SG also deaf }\end{array}$

Utterance translation: 'Yes, my sister is also deaf.'

\begin{tabular}{|c|c|c|c|c|c|c|}
\hline & $00: 00: 41.000$ & & $0: 41.500$ & $00: 00: 42.00$ & & $00: 00: 42.500$ \\
\hline \multicolumn{7}{|l|}{ LH: } \\
\hline \multicolumn{2}{|c|}{ RH: PT:1 } & \multicolumn{2}{|c|}{ ZUS-B } & \multirow[t]{2}{*}{ PT } & \multicolumn{2}{|l|}{ DOOF-A } \\
\hline MA: & 'ja' & 'mijn' & |'zuster' & & 'ook & |doof \\
\hline $\mathrm{T}:$ & M-add & $M$ & $M$ & $M-a$ & M-add & M \\
\hline
\end{tabular}

Finally, we found a few cases where Dutch idiom is used in a signed sentence. Example (5.16) is such a case, where the Dutch idiom sterk in je schoenen staan ('being confident, credible') is mouthed together with a quite literal signed translation. Multiword idiomatic expressions are very rare in signed languages (Johnston \& Ferrara, 2012), and NGT is no exception; this example clearly stands out as a Dutch expression.

(5.16) ID-gloss: PT BROTHER STAND STRONG PT SHOE PALM-UP Mouth: mijn broer stond sterk in $z^{\prime} n$ schoen-en Mouth gloss: I.POSS brother stand.PST strong in he.POSS shoe-PL

Utterance translation: 'My brother was very confident.'

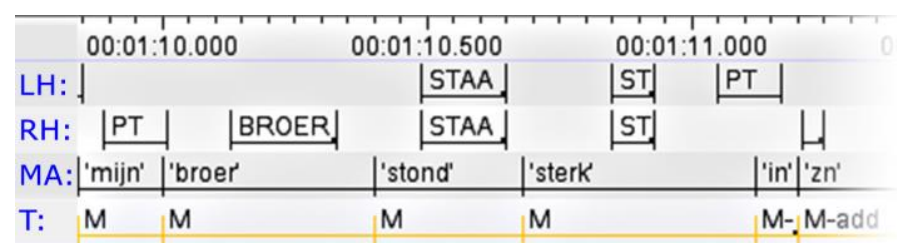

(cont.):

\begin{tabular}{|c|c|c|c|}
\hline 0 & 00:01:11.500 & $00: 01: 12.000$ & $00: 01: 12$ \\
\hline 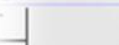 & SCHOEN- & PO & \\
\hline $\mathrm{L}$ & SCHOEN- & PO & \\
\hline 'in'|'zn' & 'schoenen' & & \\
\hline$M-M-a d o$ & M & & \\
\hline
\end{tabular}




\subsection{Discussion}

We first summarise our findings (5.4.1), discuss implications for psycholinguistic models of bimodal language processing (5.4.2), then discuss implications for models of code mixing (5.4.3), and conclude with a brief discussion of the limitations of the present study and suggestions for further research (5.4.4).

\subsubsection{Summary of our findings}

We have analysed the various forms in which non-standard mouthings occur in NGT dialogue by and between native signers with no hearing people around. We found that non-standard mouthings occur in roughly $12 \%$ of all utterances in our sample. Most of these non-standard mouthings are added mouthings: they do not have a manual correlate but occur during manual activity. All word classes are present; conjunctions, verbs, adverbs and prepositions form the largest classes. Solo mouthings, also without a manual correlate but with the hands in rest position, and specifying mouthings, that specify the meaning of a co-temporal manual sign, take up smaller parts in our sample. The use of non-standard mouthings is a pervasive communication strategy, used by the great majority of signers in the sample (36 out of our 40 signers). It is important to note that they are not speech errors: no communication problems were observed, and signers did not ask their interlocutors for clarification. In many cases (e.g. with the use of spatial prepositions, wh-question words, negation), there are lexical ways in NGT to convey the message using manual signs, but signers use added mouthings instead. Moreover, when we take all sources together, we find that manual, mouthed and other non-manual information leads to semantically coherent utterances where signers exploit the possibilities each language gives them.

We can speculate about why deaf signers use non-standard mouthings when signing among each other. All signers from deaf communities can be regarded as bilingual (Bank, Crasborn \& Van Hout, under review), and it is safe to assume, given the enormous number of mouthing present in NGT, that both spoken and signed languages are active in the signer's brain. We offer three non-competing hypotheses as to why signers do use mouthings:

Efficiency. With two manual articulators at their disposal, signers are already able to convey meaning in a condensed form through the use of simultaneous constructions (Vermeerbergen, Leeson, \& Crasborn, 2007). Adding the mouth as a 
third articulator would broaden the range of possibilities and deliver more information in a compact way, such as happens with the use of specifying mouthings.

Ease of articulation. Oral articulators are smaller and therefore easier to move than the rather large articulators like hands and arms (Crasborn, 2012). Once both languages are active in the brain of the signer, it is easy to slip in a word or two without hampering the information stream (Emmorey et al., 2012). This could lead to added and solo mouthings.

Creativity. While it does not happen all the time during everyday signing, signers do make creative use of the possibilities that having multiple articulators give them, in the sense of not only producing redundant information. Making use of a third articulator to produce code-blends (Emmorey, Borinstein et al., 2005, 2008), combining sign language with elements from spoken language, is another possibility for creative language use.

In section 5.3.6 we looked at the use of Dutch word order, and found only a small number of sentences that could be classified as structurally Dutch. However, we only looked at the sentence level; further research may investigate 'mouthings as Dutch sentences' more thoroughly - do they perhaps occur more often than we have been able to establish in the present study? Moreover, it may be that an investigation at the constituent level may reveal more Dutch structures in NGT constituents.

\subsubsection{Implications for bimodal bilingual processing}

From the point of view of bilingual processing, our findings suggest that there is more complex bilingual processing going on than simply the lexical co-activation of Dutch word forms with NGT signs, such as is likely taking place in the omnipresent use of standard mouthings (see also Bank et al., under review). The use of added mouthings, and especially the use of Dutch function words, suggests that Dutch morphosyntactic constructions or representations are activated in the production of NGT sentences. This leads to the production of a scale of Dutch words that sometimes fits nicely with the word order and morphology of NGT, and sometimes produces mixed utterances with elements such as copulas or non-spatial prepositions unknown in the grammar of NGT.

A large variety of signers use non-standard mouthings, suggesting that they expect their interlocutors to process it. A perceiving signer maintains a relatively steady gaze toward the producing signer (Siple, 1978). More precise, the perceiver gazes at the other signer's face, where beginning (L2) signers tend to fixate on (the 
area around) the mouth, and native signers tend to fixate on (the area around) the eyes (in ASL, Emmorey, Thompson, \& Colvin, 2009). However, ASL has a longstanding reputation of hardly featuring any mouthings (but see Nadolske \& Rosenstock, 2007, who argue otherwise). It would be interesting to see whether native NGT signers fix their gaze more upon the mouth than what Emmorey et al. found for ASL, as this could indicate a difference the way the two languages are processed. This has yet to be investigated by eye-tracking studies.

\subsubsection{Implications for models of code mixing}

If we compare the combinations of words and signs found in our data with Muysken's (2013) taxonomy of code-mixing, we see some similarities and some differences. We found a few instances that could be argued to be covered by Muysken's alternation, where NGT structure was temporarily replaced with spoken Dutch structure (e.g. examples 5.3 and 5.4). There were also only a few cases of congruent lexicalisation, where the grammatical structures of both languages are shared (e.g. example 5.15). We found no occurrences of backflagging in our data. The use of standard mouthings could be seen as insertion, although there is no replacement of items from the matrix language. A better fitting term may be 'addition' (see Bank et al., 2011), since there is no real switch from one language to another due to the bimodality of the utterances. Added mouthings and solo mouthings may be classified as insertion (or addition) as well, as they leave the structure of the matrix language intact. An interesting difference with insertion in spoken languages is that in the latter, the inserted elements are overwhelmingly nouns (Myers-Scotton, 2006), where we typically found copulas, conjunctions, prepositions, and negations, that is, functional elements.

Muysken $(2000,2013)$ uses code-mixing as a neutral term to cover concepts like code-switching and (nonce-)borrowing. Essentially, all categories described in his model (insertion, alternation, congruent lexicalisation, and backflagging) assume a single articulatory channel, resulting in a model where, in any given string, there is only one active language observable at a time. While the term code-blending partly resolves this issue by describing (bimodal) simultaneous utterances, it is too generic to classify bimodal code-mixing strategies. It seems that adding 'addition' as a fifth strategy to Muysken's model would serve to resolve this issue.

Terpstra and Schermer (2006) discuss sign-supported speech in terms of a number of variants on a continuum between NGT and NmG. These variants are not seen as three different language varieties, but as illustrations of types of variation that 
occur when NGT elements are integrated in Dutch spoken utterances. They are noncategorical, and overlapping. On the basis of the results of the present study, we propose that even the 'purest' form of sign language in the Netherlands has similar language contact characteristics. Dutch elements are integrated in various ways and to various extents into the matrix language NGT.

At one end of the continuum there are cases where standard mouthings are used in sentences that show no other influence of spoken Dutch. This would be the 'pure' NGT side of the continuum. Moving along the continuum, we find constructions where NGT word order is still maintained, but Dutch spoken elements are also inserted between manual signs (added and solo mouthings) and semantically non-congruent Dutch words (specifying mouthings) are used with manual signs. At the other end, we find constructions where Dutch word order influences the NGT word order, or where strings of Dutch words are articulated forming Dutch phrases or sentences, without any manual signs (sequences of multiple solo mouthings). Just as for the NmG continuum, these variants have no special ideolectal or variant status, but are meant to illustrate the types of code-blending, code-mixing, and codeswitching that occur along the continuum.

Although the two continua appear to be mirror images of each other, together forming one single continuum, there is one key property that differentiates the two: the nearly omnipresent use of voice in the NmG continuum, and the near-absent use of voice (or ideolectal variation in this regard) in the NGT continuum. For this reason, we suggest that the two are indeed better seen as two separate scales. In the first, Dutch is the matrix language, in the second, NGT is the matrix language.

\subsection{Conclusion}

There are many ways to get a message across, even within a single language. For most if not all of the examples we found, the use of non-standard Dutch mouthings is just an alternative way of expressing something that could also be expressed by manual signs and non-manual expressions alone. As we found in earlier studies, Dutch mouthings form an omnipresent complement to more 'native' manual-visual forms. In a substantial minority of sentences in our data set, Dutch is used in a way that cannot be seen as strictly linked to individual manual signs. Dutch lexical elements, therefore, both standard and non-standard, are a paramount feature of everyday sign 
language use and understanding. When it is not sanctioned, frequent mixing is indeed a quite viable mode of communication. 
114 | Chapter 5: Bimodal code-mixing: speech supported signing is the norm for deaf NGT signers

\section{Appendix}

Here follows a list of the locations where the examples can be found. The numbers correspond to those of the examples in the paper; the links point to videoclips where the pictures of both signers are combined.

(5.1) https://corpus1.mpi.nl/qfs1/media-archive/NGT/Public/Media/CNGT1792.mpg (at time point 01:35.900, signer on the right)

(5.2) https://corpus1.mpi.nl/qfs1/media-archive/NGT/Public/Media/CNGT0250.mpg (at time point 00:09.800, signer on the right)

(5.3) https://corpus1.mpi.nl/qfs1/media-archive/NGT/Public/Media/CNGT0170.mpg (at time point 02:34.100, signer on the left)

(5.4) https://corpus1.mpi.nl/qfs1/media-archive/NGT/Public/Media/CNGT0295.mpg (at time point 03:10.200, signer on the left)

(5.5) https://corpus1.mpi.nl/qfs1/media-archive/NGT/Public/Media/CNGT0098.mpg (at time point 04:21.650, signer on the left)

(5.6) https://corpus1.mpi.nl/qfs1/media-archive/NGT/Public/Media/CNGT0098.mpg (at time point 03:03.700, signer on the right)

(5.7) https://corpus1.mpi.nl/qfs1/media-archive/NGT/Public/Media/CNGT0137.mpg (at time point 02:25.050, signer on the left)

(5.8) not publicly available

(5.9) https://corpus1.mpi.nl/qfs1/media-archive/NGT/Public/Media/CNGT0098.mpg (at time point 00:17.220, signer on the right)

(5.10) https://corpus1.mpi.nl/qfs1/media-archive/NGT/Public/Media/CNGT0531.mpg (at time point 01:16.300, signer on the left)

(5.11) https://corpus1.mpi.nl/qfs1/media-archive/NGT/Public/Media/CNGT1791.mpg (at time point 01:00.440, signer on the right)

(5.12) https://corpus1.mpi.nl/qfs1/media-archive/NGT/Public/Media/CNGT0335.mpg (at time point 02:45.400, signer on the right)

(5.13) not publicly available

(5.14) https://corpus1.mpi.nl/qfs1/media-archive/NGT/Public/Media/CNGT0170.mpg (at time point 05:06.600, signer on the left)

(5.15) https://corpus1.mpi.nl/qfs1/media-archive/NGT/Public/Media/CNGT0008.mpg (at time point 00:40.820, signer on the right)

(5.16) https://corpus1.mpi.nl/qfs1/media-archive/NGT/Public/Media/CNGT0432.mpg (at time point 01:09.860, signer on the left) 


\section{Chapter 6: Discussion}

T

he findings of the previous four studies lead us to conclude that all NGT signers use mouthings to communicate or to support their communicative efforts. What does this mean for the sign language system? Traditional grammars of spoken languages do not include gesturing as a relevant part of grammar. The corresponding conclusion for sign languages appears to be attractive: mouthings are not a constructive or essential part of their grammars. However, just as gesture studies in the past twenty years have emphasised the close link between nonverbal behaviour and the linguistic system of hearing speakers, we would like to emphasise that sign and spoken elements are inextricably linked in deaf communication.

Our conclusions on how signs and mouthings intertwine are based on spontaneous, concrete language behaviour, available in the form of corpus data. The corpus studies we conducted and which were reported in the previous chapters show how omnipresent their interaction is, in complex structures of adding and spreading where they may be complementary but are more typically redundant. 
In this final chapter, we will first summarise what we found in the previous chapters (6.1) and discuss the implications of our findings (6.2). We will then suggest possible directions for further research (6.3) and offer some methodological observations (6.4), before wrapping up (6.5).

\subsection{What we found}

The general aim of this thesis was to gain more insight into how Dutch spoken language - in the form of mouthings - combines with NGT. We conducted four studies on the Corpus NGT. First, we looked at variation in the use of Dutch lexical items occurring as mouthings. In investigating 20 highly frequent signs in the Corpus NGT, we found that the majority of these signs co-occur with a semantically corresponding mouthing: $60 \%$ of the signs occur with a standard mouthing with over $75 \%$ of their tokens. On average, the percentage of standard mouthings co-occurring with signs is $73 \%$; only five signs have percentages lower than $50 \%$. There seems to be no word class specific pattern, as high percentages for standard mouthings are found for verbs, nouns and adverbs/adjectives alike. Consequently we can conclude that variation in Dutch lexical items for these highly frequent signs is mainly confined to the same semantic field as the standard mouthing. For two signs (SCHOOL and UNDERSTAND) this was the only combination we found, suggesting that for these signs the mouthing is firmly established and may have become part of the lexical specification of the sign. Semantically non-corresponding mouthings did occur, albeit in marginal numbers. Temporal reduction of mouthings appears to be more common; the stressed syllable remains visible at all times, while realisation of unstressed syllables of the Dutch words is variable. We argued that this variability is in support of the idea of code-blending signed and spoken language. A different kind of variation, one that frequently occurred within signs, was the possibility for a sign to either cooccur with a mouthing or with a mouth gesture. This type of variation occurred in all word classes in our sample: nouns, verbs, adverbs and adjectives. On average, signs co-occur with mouth gestures in $17 \%$ of their tokens.

The second study concerned the spreading of mouthings from a source sign to neighbouring signs. We replicated the results of Crasborn, Van der Kooij, Waters, Woll, \& Mesch (2008) using a much larger and more natural data set, and confirmed that the spreading of mouthings over one or more adjacent signs is common among 
deaf native signers. In a sample of 5929 mouthings, we found that $14 \%$ spread over one or more adjacent signs. Spreading usually occurs progressively over one adjacent sign (94\%), such as SCHOOL GO with the single accompanying mouthing school, meaning 'to go to school'. However, regressive spreading was also reported (5\%), as well as spreading over multiple signs (6\%), and both types of spreading are by no means idiosyncratic phenomena. We also found that the majority of spreading mouthings (58\%) extend over a pointing sign, thereby possibly demarcating short prosodic domains, such as the prosodic word (Sandler, 1999). We found no effects for age, region, gender or nativeness. We also looked at the length of spreading mouthings in relation to non-spreading mouthings, finding that the former are significantly longer, both in number of syllables and in milliseconds. The question remains, however: does spreading happen because the signs they co-occur with are so short, or because the mouthings themselves are so long?

Next, we looked at the prominence of mouthings. We started from the assumption that older signers use more mouthings than younger signers, an idea based on the changes in deaf education in recent decades, from oralism to more sign oriented instruction. We investigated the extent to which mouthings co-occur in NGT, exploring whether any differences could be explained with sociolinguistic variation such as age, gender, nativeness, region, and highest level of education. We analysed over 10,000 mouth actions and found that around $83 \%$ of them were mouthings - mouthings are truly ubiquitous. Although the individual variation was large, even for the lower end of the signer range more than $65 \%$ of their mouth actions are mouthings. The high individual variation may contribute to the fact that we did not find sociolinguistic differences for variables like gender, age, nativeness and region. This is a notable result in itself: the frequency of the use of mouthings does not provide any indication of a signer's social background, and neither does it serve to distinguish native signers from non-native signers. The only significant difference we found was between the highest educated groups and the lowest educated groups - but the effect was small, and none of the groups in between differed significantly from any other. Even the highest educated signers, the group with the lowest proportion of mouthings, had an average of $80 \%$ mouthings out of all mouth actions. This leads to the conclusion that deaf native signers of NGT are effectively bimodal bilingual in any signed interaction, even in a maximally deaf context.

In the final study, we looked beyond the sign or word level, to investigate to what extent and in what ways additional Dutch words trickle into signed sentences. 
We discerned three types of mouthings, each adding information to an utterance without a manual equivalent being present: solo mouthings that occur while the hands are in rest position, added mouthings that occur during transitional movement of the hands or during signs that already have their own mouth action, and specifying mouthings accompanying a sign and modifying a its meaning. We showed that these added mouthings are not limited to function words, but include content words as well. Most added mouthings convey concepts or functions that could be expressed manually instead, but signers use the possibilities of their bimodal bilingual linguistic competence to their advance. Although added mouthings rarely lead to fully grammatical Dutch sentences, it is evidently a form of code-blending that is more complex than the lexical co-activation of Dutch words with NGT signs. We suggest that there exists no NGT without these forms of language contact; rather, NGT is a continuum that has NGT as its matrix language, with various degrees of Dutch blending in. There is no side of the spectrum where spoken Dutch is absent.

\subsection{What we learned: implications of our findings}

\subsubsection{Linguistic status of mouthings}

We have shown that mouthings form an integral part of deaf communication in the Netherlands. The ubiquity of mouthings in our dataset - a dataset containing deaf native signers in natural conversation, no hearing people present - suggests that deaf signers cannot do without them. But mouthings as a part of communication may be something different than mouthings as a part of language. We do not have a definitive answer to the overarching question we posed about the linguistic (let alone psycholinguistic) status of mouthings in chapter 1: are they a form of code-mixing where signers may freely and creatively combine signs and mouthings, or have they become a part of the sign language lexicon as a phonological specification of a manual sign? Still, the most striking finding is that there is no deaf communication in the Netherlands without mouthings. There may be occasional NGT sentences that do not manifest any presence of Dutch mouthings, but it is clearly the exception rather than the rule.

The findings from our study on variation (chapter 2) suggested that for some signs it may be the case that mouthings are indeed specified in the lexicon. We also 
found that the articulation of mouthings was variable because of temporal reduction. We considered this as support for the idea of code-blending signed and spoken language, because there appears to be no fixed production form of these mouthings. However, some models of processing reduced speech in spoken language assume that multiple forms of pronunciation can be stored in the mental lexicon (for an overview, see Ernestus \& Baayen, 2011; Ernestus \& Warner, 2011).

The findings from our study on added mouthings clearly show that there is no strict linking between the hands and the mouth, in that words from the spoken language are added to the signing stream. The variability could be taken as an argument for non-specification at the level of lexical representation; hence, they could be considered as online code-blending. If, alternatively, there were to be a lexical representation of mouthings alongside the manual information, two distinct types of mouthings would have to be contrasted: one specified in the sign language lexicon, the other code-blended from spoken language (with deletion of the mouthing part of the lexical form). We would like to propose, therefore, that mouthings are not specified in the NGT lexicon, but are always instances of code-blends. The mechanisms behind this kind of mixing are yet to be fully understood.

We thus agree with Hohenberger and Happ (2001), who argued that mouthings are not part of the lexical description of a sign. Hohenberger and Happ, however, also reasoned that mouthings are not linguistically relevant and "may as well be absent" (2001: 157); this latter suggestion is unsupported by cross-linguistic evidence (but see De Vos \& Zeshan, 2012, on Kata Kolok), and just like Mohr (2011) argued for Irish Sign Language, we find it hard to believe that this could be the case for NGT.

There is something to be said for the idea put forward by Ebbinghaus and Heßmann (2001), who reject both lexical specification (because of the variation in mouthings, or 'unpredictability' in their words) and code-mixing (because that implies a mouthingsless state of the sign language where mouthings could be mixed in) as explanations for mouthings. Instead, they believe that sign language is a multidimensional form of communication. This has likewise been argued for hearing interaction, combining speech and non-verbal behaviour: Enfield (2009) and Kendon $(2011,2013)$ speak of hearing interaction in terms of 'composite utterances' that encompass all communicatively relevant (visible, audible, or tactile) behaviour. Thus, there is common ground between the idea of composite utterances and the idea of 'speech-supported sign' put forward in chapter 5. 


\subsubsection{Code-mixing and code-blending}

Muysken (2013), reformulating and expanding the taxonomy of code-mixing presented in Muysken (2000), describes four strategies of code-mixing observed in spoken language users. The first three strategies are insertion of lexical material from one language into the matrix or base language (the L1); alternation between structures from languages, combining fragments from different languages independently of the grammars involved; and congruent lexicalization of material from different lexical inventories into a grammatical structure that shares properties of both L1 and L2. A fourth possible strategy is backflagging, where the matrix language is not the L1 but L2, and the grammatical and lexical properties of the L2 are marked with flagging elements from the L1.

How do our findings on mouthings fit with this typology? One of the problems of fitting in code-mixed signed and spoken language is that it is difficult to establish which language exactly is the L1 and which the L2, given that they will often be acquired simultaneously (cf. Plaza-Pust, 2012). Sign language may be the most readily accessible, but for most deaf children sign language input is less than optimal and certainly not as omnipresent as the spoken language. Having said that, we can try to place our findings in Muysken's (2013) model. We found in chapter 5 that the base language is overwhelmingly NGT, with abundant insertion of lexical elements and also occasional congruent lexicalisation and alternation with Dutch. We argue, therefore, that NGT signers mostly use the insertion strategy. We also argue that the term 'addition' would fit better for this strategy of code-mixing than 'insertion', because the element that is inserted into the matrix language does not replace it (as is the case in spoken language), but appears simultaneously. This would seem a useful addition to Muysken's model.

Throughout this thesis, we have used the term code-blending to refer to the simultaneous occurrence of codes in two modalities, spoken and signed. The term was coined by Emmorey, Borinstein, and Thompson (2005), to describe the kind of codemixing found with hearing signers, who were mixing spoken English and ASL. We adopted the term to also cover the mixing of silently mouthed spoken language and signed language. Code-blending as a general term sidesteps the need for a distinction between a matrix and a guest language, or between L1 and L2. Because the blended codes reside in separate modalities, either of the two can take the role of the matrix language, depending on the skills of the signer and on the needs of his or her interlocutor. Code-blending is a useful term to distinguish bimodal from unimodal 
code-mixing. Just like code-mixing, it serves as an umbrella term for a range of mixing (blending) strategies that signers have at their disposal.

\subsection{What we suggest for further research}

\subsubsection{Mouthing research}

We have established frequencies of mouthings in their various forms, and split certain forms into smaller groups (such as the added mouthing category). We looked at ratios between mouthings and mouth actions and between different groups of signers. After having done all that, we concluded that it is likely that mouthings are not specified in the NGT lexicon, but instead that they are instances of code-blends, creating composite utterances that in the general sense of using all available resources are not unlike composite utterances in spoken language where manual gestures, other nonverbal behaviour, and speech are combined.

What does this mean for mouthing research? From a sign language perspective, the absence of a lexical specification in the sign lexicon may render mouthings less interesting, because they do not seem to be part of the language proper. However, we think that the interaction between the hands and the mouth provides many interesting points of departure for further research. It is still relevant to study the semantic and morphosyntactic function of mouthings in the composite utterances, things that have been underexposed in our studies. It seems clear that some mouthings, the specifying kind, have a disambiguating function such as the mouthing 'brother' and 'sister' with the sign SIBLING, or the mouthing 'group', 'family' or 'class' with the sign GROUP. The latter sign was the only one in our variation study with a clear disambiguating function. A replication of this study with more signs, including function words and less frequent signs, may reveal more about the specifics of disambiguation.

One aspect of the interaction between the signers in the corpus that we did not study is whether variation, spreading, or the number of mouthings change in the course of a recording session. Although the signer dyads were already good acquaintances and should have felt comfortable in signing with each other, it is possible that they were initially a bit uneasy in the recording set-up (at the university, with cameras and light) but grew more confident in the course of the session. 
Although at odds with our general conclusion that mouthings are omnipresent, it could be that details of the amount of code blending still is influenced by nonlinguistic factors such as emotional state. Currently, the number of annotations in the corpus is not sufficient to systematically compare between the beginning and the end of the sessions for the way signers use mouthings, but future research could look into this.

Now we have established how omnipresent mouthings are, we can look in greater detail to the functions of mouthings as already outlined by Schermer (1990). A corpus study, however, is not the most obvious method for this kind of research, which is better achieved with psycho- and neurolinguistic tests. One possible research direction is to study the influence of salience of information: will there be fewer mouthings when there is repeated reference to a referent? It has been argued for both speech, gesture, and sign that they are likely to appear in reduced form when repeated again later in the same discourse (Hoetjes, Koolen, Goudbeek, Krahmer, \& Swerts, 2011; Hoetjes, Krahmer, \& Swerts, 2012). And what about mouthings in for example family situations, where there is lots of context for a meaning to be derived from?

A final issue concerns the mixing of Dutch morphosyntactic structures into the communication stream. We have seen that mouthed verbs and nouns can sometimes be inflected for time, person and number. Under what circumstances does this insertion of syntactic information appear, and how do NGT and Dutch interact in this process? Are there types of inflection that appear to be easier to mix than others? Are there specific grammatical contexts in NGT that promote or elicit inflected mouthings? These questions, too, have never been investigated (for any sign language), to our knowledge.

\subsubsection{Individual differences}

One thing we found in every study we conducted, was that there were always large differences between signers: in variation of the use of mouthings or mouth gestures, the use of spreadings, mouthing to mouth action ratio, and so forth. This was most prominent in chapter 3, where we looked at social parameters like gender, age, nativeness, region, and education. There were virtually no between-group differences, but the within-group variation was quite large, so that all groups performed in comparable ways. Other researchers have also observed large amounts of variability in their data on mouthings (e.g. Hohenberger \& Happ, 2001; Keller, 2001; Zeshan, 2001), and the study of individual differences is currently a trendy topic in 
psycholinguistics (see contributions to Roberts \& Meyer, 2012). Is the variation between users similar to, for instance, individual differences in word pronunciation in spoken language? Does this have consequences for the way signers have to accommodate to their interlocutors? It would also be interesting to see if variation in mouthings is on a par with other between-signer variation like in signing speed or in sign vocabulary size, for instance. To date, there has been no large-scale sociolinguistic study on NGT that such a study could build on, unfortunately.

\subsubsection{Mouth gestures}

In chapter 2, we found that signers (whether or not subconsciously) choose between a mouthing and a mouth gesture while articulating a sign. It has been suggested that mouth gestures take precedence over mouthings, because mouth gestures convey obligatory syntactic and semantic information. (e.g. Sutton-Spence \& Woll, 1999; Hohenberger \& Happ, 2001). This seems certainly the case for adverbial or adjective (type A) mouth gestures, as these add meaning to the sign they accompany, such as indicating the manner in which an action is performed, or the size of an object. Therefore, they may receive precedence over redundant mouthings. Similar arguments could be made for the other types of mouth actions (E, 4, and W; see Crasborn, Van der Kooij et al., 2008).

Although we did not further analyse mouth gestures beyond labelling them for the appropriate categories proposed by Crasborn, Van der Kooij et al. (2008), we found that the labelling itself was sometimes problematic. There are many mouth actions that are not easily placed in one of the AE4W categories - they do not seem to have an independent meaning nor do they seem to be lexically associated. These properties indicate them as being $\mathrm{W}$-type mouth gestures, but they don't fit the description of W-type as in "Mouth activity is seen in the context of whole-face activity" (Crasborn, Van der Kooij et al., 2008: 50). Future research could look into these mouth gestures and determine their linguistic status. Are they perhaps part of the linguistic system after all, rather than part of an affective facial expression? Or are these actually co-sign mouth gestures, not unlike co-speech gesture with spoken language? Finding the answers to these questions could shed some light on another question arising from our findings. As we said, we found it hard at times to correctly label mouth gestures with non-salient meanings. Yet these mouth gestures do occur and seem to take precedence over mouthings. Is spoken language periodically less 
activated during these instances? What circumstances affect that choice, whether subconsciously made or not?

\subsubsection{Sociolinguistic variation}

Studies on the sociolinguistics of sign languages have shown that standard sociolinguistic variables like age, gender, region, and social class manifest themselves in different aspects of sign languages like ASL, BSL, Auslan, and NGT. Regional variation has been reported for, for instance, lexical differences in BSL (Sutton-Spence \& Woll, 1999) and NGT (Schermer, 2003), or for fingerspelling in Auslan (Schembri \& Johnston, 2007). Variation according to age and social class was reported for handshape in ASL by Lucas, Bayley, and Valli (2001); gender differences were found for turn-taking patterns in BSL (Coates \& Sutton-Spence, 2001).

We did not find a lot of sociolinguistic variation in our data. It is possible that our corpus was too small, or too diverse. A replication of our studies with the same number of signers who differ in only one sociolinguistic variable could possibly result in a different outcome. And as we said above, it may be that individual differences simply outnumber group differences. Moreover, a quite different explanation for the lack of variation could be that we did not tap into the right dependent variables in our studies. Looking in greater phonetic detail at the articulatory form, using methods such as ultrasound (Scobbie, Punnoose, \& Khattab, 2013) or electropalatography (Baltazani \& Nicolaidis, 2013), might help us in distinguishing generations of deaf people or different social groups.

\subsubsection{Perception}

Since the source of our data is a corpus, we have taken a production perspective in all of our studies. But what is the role of mouthings in perception and processing? To what extent do mouthings improve or contribute to recognition of (composite) lexical items? Eye gaze studies have shown that the mouth falls in the centre of the visual field for sign language interlocutors: they tend to look at the face and not at the hands (McCullough \& Emmorey, 1996; Siple, 1978). Therefore, the visual information of the mouthings is accessible in great detail. Although there is little research (but see Capek et al., 2008; Woll, 2012), it is a common experience that many deaf signers are excellent lipreaders. Future research could explore whether lexical recognition is improved by mouthings or not, and to which extent added mouthings are perceived by signers and play a role in the interpretation of utterances. 
Aside from lexical content in the form of phonemic distinctions, the speech signal of hearing speakers also carries many prosodic and paralinguistic distinctions (Gussenhoven, 2004; Lehiste, 1970; Scherer, 1986, 2003). We do not know at present whether such detail is actually present in the mouthings of deaf signers: mouthings have always been approached as individual lexical items, their phonetic variation studied in terms of phonetic reduction but not in terms of other phonetic variables. Given the fluency of signers in the spoken language, it is not unlikely that some prosodic and paralinguistic features are also present in the (visual) signal. These phonetic properties also merit investigation from a perception perspective.

\subsection{Some methodological notes}

The Corpus NGT is the best effort to date to compile a representative set of natural sign language data in the Netherlands. It is among the first and the largest sign language corpora in the world, and it is available online for any researcher. It can be searched using the ANNEX tool (tla.mpi.nl/tools/tla tools/annex/; see also Berck \& Russel, 2006), which has similar search capabilities as the offline ELAN software. However, the corpus inevitably has its flaws, in part because of its ground-breaking position. Some of these flaws have affected our studies. The first sets of gloss annotations were not firmly based on a lexicon, and only part of the material was validated by a second annotator. The lack of adequate guidelines for many aspects of the glossing, combined with insufficient monitoring, resulted in annotations that were of variable quality (Crasborn \& De Meijer, 2012; Crasborn \& Zwitserlood, 2008a). In the course of the last few years, a lexicon of ID-glosses has been constructed (Crasborn \& De Meijer, 2012), which has been integrated into the ELAN annotation documents in the form of an External Controlled Vocabulary (Crasborn, Hulsbosch, \& Sloetjes, 2012). This in turn has been used for the revision of the glosses in the present project.

A relatively recent addition to ELAN (introduced in the release of version 4.1.0 in April 2011) is the possibility to create annotations aligned with video frames. The frame rate of the corpus videos is 25 frames per second, or one frame per $40 \mathrm{~ms}$. Annotations are created by clicking and dragging the mouse over the desired part of the timeline; release 4.1.0 made it possible to automatically align such a selection with the video frames by rounding the time values for the new annotation to the start/end 
of the video frames involved. Before version 4.1.0, all annotations were created as they were selected, accurate to the millisecond. This resulted (unwillingly and unknowingly) in the creation of sometimes meaningless annotation durations of less than $40 \mathrm{~ms}$, sometimes also crossing frame boundaries. We suggest that efforts should be made to clean up misalignments to make annotation boundaries meaningful.

A partially related issue is that of temporal resolution. The current recordings in the Corpus NGT were made at a frame rate of 25 frames per second. While this is in principle enough to comfortably discern all manual activity, the mouth is faster than the hands are (Bellugi \& Fisher, 1972). In normal speech, speaking rate is almost 6 syllables per second (e.g. Cucchiarini, Strik, \& Boves, 2002), or $167 \mathrm{~ms}$ per syllable, roughly 4 video frames per syllable. This means that onset, nucleus and coda are each displayed for about one frame. For speechreading at normal speed this appears to be enough, but when a closer look is required, uncertainty about mouth articulation increases rapidly. If future recordings for the corpus were made in a higher temporal resolution, future work on mouth actions would benefit enormously, for instance to study the alignment of mouth and hand articulations in more detail to see how the syllable structures of signs line up with mouth movements.

A final, quite different, suggestion for the creation of future sign language corpora would be the inclusion of recordings where signers interact with a variety of interlocutors. One of the limiting factors of the current corpus is that all participants were recorded in fixed dyads, the interlocutors being good acquaintances. This setup ensured that the signers felt comfortable with each other and thus didn't feel inhibited (which should result in more natural signing). However, it does have the drawback that we cannot study a signer's adaptation to their interlocutors. It would be relevant to study how a signer's mouthing behaviour varies depending on the interlocutor, as it could reveal the adaptive mechanisms in signer interaction.

\subsection{Conclusion}

We have shown that Dutch spoken language has a major influence on NGT. Mouthings from spoken Dutch are ubiquitous. Given this influence, we would like to argue that mouthings deserve a more prominent place in sign language research, and that they should be included in any description of signed communication, however elementary. We have no reason to believe that this will be different in other countries 
or regions that have comparable educational systems and history of deaf education, and a comparable social status of deaf people. We would therefore like to put forward the claim that our findings are transferable to other spoken/signed language combinations. At the same time, it is not to be excluded that deaf communities differ in the extent to which mouthings are combined with manual signs. That is, both the extent to which and the ways in which mouthings are combined with manual signing may well be under linguistic control. The types of composite utterances we observe in sign languages could 'grammaticalise' in different directions, given enough time.

We would like to conclude with a data related remark. The annotations that were made for this project are included in the second release of the Corpus NGT annotations, published in the second half of 2014. This will give researchers worldwide the ability to verify or falsify the claims we reported on mouthings in NGT, to compare their observations of other sign languages with ours, and to move forward our understanding of the interaction between manual signing and mouthing. 
128 


\section{References}

Ajello, R., Mazzoni, L., \& Nicolai, F. (2001). Linguistic gestures: Mouthing in Italian Sign Language. In P. Boyes Braem \& R. Sutton-Spence (Eds.), The hands are the head of the mouth. The mouth as articulator in sign languages (pp. 231-246). Hamburg: Signum Press.

Ann, J. (1993). A linguistic investigation into the relation between physiology and handshape. $\mathrm{PhD}$ dissertation. University of Arizona.

Ann, J. (2001). Bilingualism and language contact. In C. Lucas (Ed.), The sociolinguistics of sign languages (pp. 33-60). Cambridge: Cambridge University Press.

Ann, J. (2006). Frequency of occurrence and ease of articulation of sign language handshapes: The Taiwanese example. Washington, D.C.: Gallaudet University Press.

Armstrong, D. F. (1999). Original signs: Gesture, sign, and the sources of language. Washington, D.C.: Gallaudet University Press.

Armstrong, D. F., \& Wilcox, S. E. (2007). The gestural origin of language. New York: Oxford University Press.

Auer, E. T., \& Bernstein, L. E. (2007). Enhanced visual speech perception in individuals with early-onset hearing impairment. Journal of Speech, Language, and Hearing Research, 50, 1157-1165. doi:10.1044/1092-4388(2007/080)

Baker, A. E., \& Van den Bogaerde, B. (2008). Codemixing in signs and words in input to and output from children. In C. Plaza-Pust \& E. Morales Lopez (Eds.), Sign Bilingualism: language development, interaction and maintenance in sign language contact situations (pp. 1-28). Amsterdam: John Benjamins.

Baker-Shenk, C. (1983). A microanalysis of the nonmanual components of questions in American Sign Language. $\mathrm{PhD}$ dissertation. University of California. 
Baltazani, M., \& Nicolaidis, K. (2013). The many faces of /r/. In L. Spreafico \& A. Vietti (Eds.), Rhotics: new data and perspectives. Bozen-Bolzano: Bozen-Bolzano University Press.

Bank, R., Crasborn, O., \& Van Hout, R. (2011). Variation in mouth actions with manual signs in Sign Language of the Netherlands (NGT). Sign Language \& Linguistics, 14(2), 248-270. doi:10.1075/sll.14.2.02ban

Bank, R., Crasborn, O., \& Van Hout, R. (2013). Alignment of two languages: The spreading of mouthings in Sign Language of the Netherlands. International Journal of Bilingualism. Published online by Sage, May 3, 2013. doi:10.1177/1367006913484991

Bank, R., Crasborn, O., \& Van Hout, R. (under review). The prominence of spoken language elements in a sign language.

Bellugi, U., \& Fischer, S. (1972). A comparison of sign language and spoken language. Cognition, 1(2-3), 173-200. doi:10.1016/0010-0277(72)90018-2

Berck, P., \& Russel, A. (2006). ANNEX - a web-based Framework for Exploiting Annotated Media Resources. In Proceedings of the 5th International Conference on Language Resources and Evaluation (LREC) (pp. 1552-1555). Genoa. Retrieved from http://www.lrec-conf.org/proceedings/lrec2006/pdf/139_pdf.pdf

Bergman, B., \& Wallin, L. (2001). A preliminary analysis of visual mouth segments in Swedish Sign Language. In P. Boyes Braem \& R. Sutton-Spence (Eds.), The hands are the head of the mouth. The mouth as articulator in sign languages (pp. 51-68). Hamburg: Signum Press.

Bernstein, L. E., Auer, E. T., \& Tucker, P. E. (2001). Enhanced Speechreading in Deaf Adults: Can Short-Term Training/Practice Close the Gap for Hearing Adults? Journal of Speech, Language, and Hearing Research, 44, 5-18. doi:10.1044/1092-4388(2001/001)

Bernstein, L. E., Tucker, P. E., \& Demorest, M. E. (2000). Speech perception without hearing. Perception \& Psychophysics, 62(2), 233-252. doi:10.3758/BF03205546

Bos, H. (1990). Person and location marking in SLN. Some implications of a spatially expressed syntactic system. In S. Prillwitz \& T. Vollhaber (Eds.), Current Trends in European Sign Language Research. Proceedings of the 3rd European Congress on Sign Language Research, Hamburg, July 26-29, 1989 (pp. 231-248). Hamburg: Signum Press.

Bos, H. (1993). Agreement and pro-drop in Sign Language of the Netherlands. Linguistics in the Netherlands, 10, 37-47. doi:10.1075/avt.10.06bos

Bos, H. (1995). Pronoun copy in Sign Language of the Netherlands. In H. Bos \& T. Schermer (Eds.), Sign language research 1994. Proceedings of the Fourth European Congress on Sign Language Research (pp. 121-147). Hamburg: Signum.

Boyes Braem, P. (2001). Functions of the mouthings in the signing of Deaf early and late learners of Swiss German Sign Language. In P. Boyes Braem \& R. Sutton-Spence (Eds.), The hands are the head of the mouth. The mouth as articulator in sign languages (pp. 99-132). Hamburg: Signum Press.

Boyes Braem, P., \& Sutton-Spence, R. (Eds.). (2001). The hands are the head of the mouth. The mouth as articulator in sign languages. Hamburg: Signum Press.

Capek, C. M., MacSweeney, M., Woll, B., Waters, D., McGuire, P. K., David, A. S., Brammer, M. J., \& Campbell, R. (2008). Cortical circuits for silent speechreading in deaf and hearing people. Neuropsychologia, 46(5), 1233-1241.

doi:10.1016/j.neuropsychologia.2007.11.026 
Cappelletta, L., \& Harte, N. (2012). Phoneme-to-Viseme Mapping for Visual Speech Recognition. In Proceedings of the International Conference on Pattern Recognition, Applications and Methods (ICPRAM) (pp. 322-329).

Coates, J., \& Sutton-Spence, R. (2001). Turn-taking patterns in Deaf conversation. Journal of Sociolinguistics, 5(4), 507-529. doi:10.1111/1467-9481.00162

Crasborn, O. (2001). Phonetic implementation of phonological categories in Sign Language of the Netherlands. Utrecht: LOT.

Crasborn, O. (2006). Nonmanual structures in sign language. In K. Brown (Ed.), Encyclopedia of language and linguistics. 2nd edition (pp. 668-672). Oxford: Elsevier.

Crasborn, O. (2012). Phonetics. In R. Pfau, M. Steinbach, \& B. Woll (Eds.), Sign Language. An international handbook (HSK 37, pp. 4-20). Berlin/Boston: De Gruyter Mouton.

Crasborn, O., \& De Meijer, A. (2012). From corpus to lexicon: the creation of ID-glosses for the Corpus NGT. In O. Crasborn, E. Efthimiou, E. Fotinea, T. Hanke, J. Kristoffersen, \& J. Mesch (Eds.), Interactions between Corpus and Lexicon. Proceedings of the 5th Workshop on the Representation and Processing of Sign Languages. Language Resources and Evaluation Conference (LREC) (pp. 13-18). Retrieved from http://www.lrecconf.org/proceedings/lrec2012/workshops/24.Proceedings_SignLanguage.pdf

Crasborn, O., Hulsbosch, M., \& Sloetjes, H. (2012). Linking Corpus NGT annotations to a lexical database using open source tools ELAN and LEXUS. In O. Crasborn, E. Efthimiou, E. Fotinea, T. Hanke, J. Kristoffersen, \& J. Mesch (Eds.), Interactions between Corpus and Lexicon. Proceedings of the 5th Workshop on the Representation and Processing of Sign Languages. Language Resources and Evaluation Conference (LREC) (pp. 19-22). Retrieved from http://www.lrec-conf.org/proceedings/lrec2012 /workshops/24.Proceedings_SignLanguage.pdf

Crasborn, O., Mesch, J., Waters, D., Nonhebel, A., Van der Kooij, E., Woll, B., \& Bergman, B. (2007). Sharing sign language data online: Experiences from the $\mathrm{ECHO}$ project. International Journal of Corpus Linguistics, 12(4), 535-562. doi:10.1075/ijcl.12.4.06cra

Crasborn, O., \& Sáfár, A. (in preparation). From dominant to right. The phonetics and phonology of hand choice in Sign Language of the Netherlands. Ms. Radboud University Nijmegen.

Crasborn, O., Van der Kooij, E., \& Ros, J. (2012). On the weight of phrase-final prosodic words in a sign language. Sign Language \& Linguistics, 15(1), 11-38. doi:10.1075/sll.15.1.02cra

Crasborn, O., Van der Kooij, E., Ros, J., \& De Hoop, H. (2009). Topic agreement in NGT (Sign Language of the Netherlands). Linguistic Review, 26(2/3), 355-370. doi:10.1515/tlir.2009.013

Crasborn, O., Van der Kooij, E., Waters, D., Woll, B., \& Mesch, J. (2008). Frequency distribution and spreading behavior of different types of mouth actions in three sign languages. Sign Language \& Linguistics, 11(1), 45-67. doi:10.1075/s1\&l.11.1.04cra

Crasborn, O., \& Zwitserlood, I. (2008a). Annotation of the video data in the Corpus NGT. Retrieved from http://www.ru.nl/publish/pages/527859/corpusngt_annotationconventions.pdf 
Crasborn, O., \& Zwitserlood, I. (2008b). The Corpus NGT: an online corpus for professionals and laymen. In O. Crasborn, T. Hanke, E. Efthimiou, I. Zwitserlood, \& E. Thoutenhoofd (Eds.), Construction and exploitation of sign language corpora. Proceedings of the $3 \mathrm{rd}$ Workshop on the Representation and Processing of Sign Languages (LREC) (pp. 44-49). Paris: ELRA. Retrieved from http://www.lrec-conf.org/proceedings/lrec2008/workshops/W25_Proceedings.pdf

Crasborn, O., Zwitserlood, I., \& Ros, J. (2008). Corpus NGT. An open access digital corpus of movies with annotations of Sign Language of the Netherlands. Video corpus. Centre for Language Studies, Radboud University Nijmegen.

Cucchiarini, C., Strik, H., \& Boves, L. (2002). Quantitative assessment of second language learners' fluency: Comparisons between read and spontaneous speech. The Journal of the Acoustical Society of America, 111(6), 2862-2873. doi:10.1121/1.1471894

Cutler, A. (1998). The recognition of spoken words with variable representations. In Proceedings of the ESCA workshop on sound patterns of spontaneous speech (pp. 83-92). La Baume-les-Aix, France.

Danby, H. (1933). The Mishnah. Oxford: Oxford University Press.

De Raeve, L., Spaai, G., Uilenburg, N., Wiefferink, K., Vermeij, B., Bammens, M., Pans, R., Koppers, E., Jans, J., \& Vangeel, K. (2009). Invloed van het taalaanbod op de ontwikkeling van jonge dove kinderen met een cochleair implantaat [Influence of the language input on the development of young deaf children with a cochlear implant]. Logopedie En Foniatrie, 1, 16-23.

De Swaan, A. (2001). Words of the world: The global language system. Cambridge: Cambridge University Press.

De Vos, C. (2012). Sign-spatiality in Kata Kolok: how a village sign laguage of Bali inscribes its signing space. Nijmegen: Max Planck Institute for Psycholinguistics.

De Vos, C., \& Zeshan, U. (2012). Demographic, sociocultural and linguistic variation across rural signing communities. In U. Zeshan \& C. De Vos (Eds.), Sign Languages in Village Communities: Anthropological and Linguistic Insights (pp. 2-23). Boston/Berlin: Walter De Gruyter.

Derks, S. (2004). Doorbroken stilte. 175 jaar zorg en onderwijs voor mensen met beperkingen in horen, zien en communicatie [Broken silence. 175 years of care and education for people with disabilities in hearing, vision and communication]. Sint-Michielsgestel: Viataal.

Duncan Jr., S. (1974). On the Structure of Speaker-Auditor Interaction during Speaking Turns. Language in Society, 3(4), 161-180. doi:10.1017/S0047404500004322

Ebbinghaus, H., \& Heßmann, J. (1994). German words in German Sign Language: Do they tell us something new about sign languages? In C. J. Erting, R. C. Johnson, D. L. Smith, \& B. D. Snider (Eds.), The deaf way (pp. 399-409). Washington D.C.: Gallaudet University Press.

Ebbinghaus, H., \& Heßmann, J. (2001). Sign language as multidimensional communication: why manual signs, mouthings, and mouth gestures are three different things. In P. Boyes Braem \& R. Sutton-Spence (Eds.), The hands are the head of the mouth. The mouth as articulator in sign languages (pp. 133-151). Hamburg: Signum. 
Emmorey, K., Borinstein, H. B., \& Thompson, R. (2005). Bimodal bilingualism: Code-blending between spoken English and American Sign Language. In J. Cohen, K. T. McAlister, K. Rolstad, \& J. MacSwan (Eds.), ISB4: Proceedings of the 4th International Symposium on Bilingualism (pp. 663-673). Somerville, MA: Cascadilla Press. Retrieved from http://www.lingref.com/isb/4/051ISB4.PDF

Emmorey, K., Borinstein, H. B., Thompson, R., \& Gollan, T. H. (2008). Bimodal bilingualism. Bilingualism: Language and Cognition, 11(1), 43-61. doi:10.1017/S1366728907003203

Emmorey, K., Luk, G., Pyers, J. E., \& Bialystok, E. (2008). The source of enhanced cognitive control in bilinguals. Psychological Science, 19, 1201-1206. doi:10.1111/j.1467-9280.2008.02224.x

Emmorey, K., Petrich, J. A. F., \& Gollan, T. H. (2012). Bilingual processing of ASL-English code-blends: The consequences of accessing two lexical representations simultaneously. Journal of Memory and Language, 67, 199-210. doi:10.1016/j.jml.2012.04.005

Emmorey, K., Thompson, R., \& Colvin, R. (2009). Eye Gaze During Comprehension of American Sign Language by Native and Beginning Signers. Journal of Deaf Studies and Deaf Education, 14(2), 237-243. doi:10.1093/deafed/enn037

Enfield, N. J. (2009). The Anatomy of Meaning. Speech, Gesture, and Composite Utterances. Cambridge: Cambridge University Pres.

Ernestus, M. (2000). Voice assimilation and segment reduction in casual Dutch: a corpus-based study of the phonology-phonetics interface. Utrecht: LOT.

Ernestus, M., \& Baayen, H. (2011). Corpora and exemplars in phonology. In J. Goldsmith, J. Riggle, \& A. C. L. Yu (Eds.), The Handbook of Phonological Theory: Second edition (pp. 374-400). Oxford: Wiley-Blackwell. doi:10.1111/b.9781405157681.2011.00013.x

Ernestus, M., Baayen, H., \& Schreuder, R. (2002). The recognition of reduced word forms. Brain and Language, 81, 162-173. doi:10.1006/brln.2001.2514

Ernestus, M., \& Warner, N. (2011). An introduction to reduced pronunciation variants. Journal of Phonetics, 39(3), 253-260. doi:10.1016/S0095-4470(11)00055-6

Fenlon, J., Schembri, A., Rentelis, R., \& Cormier, K. (2013). Variation in handshape and orientation in British Sign Language: The case of the ' 1 ' hand configuration. Language \& Communication, 33(1), 69-91. doi:10.1016/j.langcom.2012.09.001

Fontana, S. (2008). Mouth actions as gesture in sign language. Gesture, 8(1), 104-123. doi:10.1075/gest.8.1.08fon

Frishberg, N., Hoiting, N., \& Slobin, D. I. (2012). Transcription. In R. Pfau, M. Steinbach, \& B. Woll (Eds.), Sign Language. An international handbook (HSK 37, pp. 1045-1075). Berlin/Boston: De Gruyter Mouton.

Groce, N. E. (1985). Everyone here spoke sign language - Hereditary deafness in Martha's Vineyard. Cambridge: Harvard University Press.

Grosjean, F. (2010). Bilingual. Life and reality. Cambridge: Harvard University Press.

Gussenhoven, C. (2004). The Phonology of Tone and Intonation. Cambridge: Cambridge University Press.

Gustason, G., Pfetzing, D., \& Zawolkow, E. (1975). Signing Exact English. Silver Spring, Maryland: Modern Sign Press.

Hermans, D., Ormel, E., \& Knoors, H. (2010). On the relation between the signing and reading skills of deaf bilinguals. International Journal of Bilingual Education and Bilingualism, 13(2), 187-199. doi:10.1080/13670050903474093 
Hiddinga, A., \& Crasborn, O. (2011). Signed languages and globalization. Language in Society, 40(04), 483-505. doi:10.1017/S0047404511000480

Higgins, D. D. (1923). How to talk to the Deaf. The language of gestures, expression, impersonation, pantomime or acting used by all peoples in all ages and everywhere. St. Louis: privately printed by the Catholic church at 1118 N. Grand Blvd. Reprinted in 1959 by Mount Carmel Guild, Archdiocese of Newark.

Hoetjes, M., Koolen, R., Goudbeek, M., Krahmer, E., \& Swerts, M. (2011). GREEBLES Greeble greeb: On Reduction in Speech and Gesture in Repeated References. In Proceedings of the 33rd Annual Conference of the Cognitive Science Society (CogSci 2011) (pp. 3250-3255). Boston. Retrieved from http://mindmodeling.org/cogsci2011/papers/0748/paper0748.pdf

Hoetjes, M., Krahmer, E., \& Swerts, M. (2012). Do repeated references result in sign reduction? In Proceedings of the 34th annual meeting of the Cognitive Science Society (CogSci 2012) (pp. 461-466). Sapporo, Japan. Retrieved from http://mindmodeling.org/cogsci2012/papers/0091/paper0091.pdf

Hohenberger, A., \& Happ, D. (2001). The linguistics primacy of signs and mouth gestures over mouthing: evidence from language production in German Sign Language. In P. Boyes Braem \& R. Sutton-Spence (Eds.), The hands are the head of the mouth. The mouth as articulator in sign languages (pp. 153-190). Hamburg, Germany: Signum Press.

Hoyer, K. (2004). The sociolinguistic situation of Finland-Swedish deaf people and their language, Finland-Swedish sign language. In M. van Herreweghe \& M. Vermeerbergen (Eds.), To the Lexicon and Beyond: Sociolinguistics in European Deaf Communities (pp. 3-23). Washington D.C.: Gallaudet University Press.

Jesse, A., \& Massaro, D. W. (2010). The temporal distribution of information in audiovisual spoken-word identification. Attention, Perception, \& Psychophysics, 72(1), 209-225. doi:10.3758/APP.72.1.209

Johnston, T. (2008). Corpus linguistics and signed languages: No lemmata, no corpus. In O. Crasborn, T. Hanke, E. D. Thoutenhoofd, I. Zwitserlood, \& E. Efthimiou (Eds.), Construction and exploitation of sign language corpora. Proceedings of the $3 r d$ Workshop on the Representation and Processing of Sign Languages (LREC) (pp. 82-87). Paris: ELRA. Retrieved from

http://www.lrec-conf.org/proceedings/lrec2008/workshops/W25_Proceedings.pdf

Johnston, T. (2010). From archive to corpus: Transcription and annotation in the creation of signed language corpora. International Journal of Corpus Linguistics, 15(1), 106-131. doi:10.1075/ijcl.15.1.05joh

Johnston, T. (2013). Auslan Corpus Annotation Guidelines. Macquairie University. Retrieved from http://media.auslan.org.au/attachments /AuslanCorpusAnnotationGuidelines_Johnston.pdf

Johnston, T., \& Ferrara, L. (2012). Lexicalization in Signed Languages: When is an Idiom not an Idiom? In C. Hart (Ed.), Selected Papers from the 3rd UK Cognitive Linguistics Conference (Vol. 1, pp. 229-248). Retrieved from

http://uk-cla.org.uk/files/proceedings/Johnston\%20and\%20Ferrara.pdf 
Kegl, J., Senghas, A., \& Coppola, M. (1999). Creation through contact: Sign language emergence and sign language change in Nicaragua. In M. DeGraff (Ed.), Language creation and language change: Creolization, diachrony, and development (pp. 179-237). Massachusetts: MIT Press.

Keller, J. (2001). Multimodal representations and the linguistic status of mouthings in German Sign language (DGS). In P. Boyes Braem \& R. Sutton-Spence (Eds.), The hands are the head of the mouth. The mouth as articulator in sign languages (pp. 191-230). Hamburg, Germany: Signum Press.

Kendon, A. (2011). Vocalisation, speech, gesture, and the language origins debate: An essay review on recent contributions. Gesture, 11(3), 349-370. doi:10.1075/gest.11.3.05ken

Kendon, A. (2013). Exploring the utterance roles of visible bodily action: A personal account. In C. Müller, A. Cienki, E. Fricke, S. H. Ladewig, D. McNeill, \& S. Teßendorf (Eds.), Body - Language - Communication (HSK 38.1, pp. 7-28). Berlin/Boston: De Gruyter Mouton.

Kerswill, P. (1996). Children, Adolescents, and Language Change. Language Variation and Change, 8, 177-202. doi:10.1017/S0954394500001137

Kita, S. (Ed.). (2003). Pointing: Where Language, Culture and Cognition Meet. New Jersey: Lawrence Erlbaum Associates, Inc.

Klima, E. S., \& Bellugi, U. (1979). The Signs of Language. Cambridge: Harvard University Press.

Knoors, H. (1992). Exploratie van de gebarenruimte: een onderzoek naar de verwerving van ruimtelijke morfosyntactische gebarentaalstructuren door dove kinderen met horende ouders [Exploration of signing space: an investigation into the acquisition of spatial morphosyntactic sign language structures by deaf children with hearing parents]. Delft: Eburon.

Knoors, H., \& Marschark, M. (2012). Language Planning for the 21st Century: Revisiting Bilingual Language Policy for Deaf Children. Journal of Deaf Studies and Deaf Education, 17(3), 291-305. doi:10.1093/deafed/ens018

Kolen, E. (2009). De tweetalige ontwikkeling van dove kinderen in de Nederlandse Gebarentaal en het Nederlands [The bilingual development of deaf children in Sign Language of the Netherlands and Dutch]. PhD dissertation. Radboud University Nijmegen.

KOMVA. (1988). Notatiesysteem voor Nederlandse gebaren [Notation system for Dutch signs]. Amsterdam: NSDSK.

KOMVA. (1989). Basis-gebarenschat: een beknopt gebarenwoordenboek [Basic sign language vocabulary: a concise sign dictionary]. Amsterdam: NSDSK.

Lane, H. (1984). When the mind hears: a history of the deaf. New York: Random House.

Leeson, L., \& Saeed, J. I. (2012). Irish Sign Language. A Cognitive Linguistic Account. Edinburgh: Edinburgh University Press.

Lehiste, I. (1970). Suprasegmentals. Cambridge, MA: MIT Press.

LeMaster, B. (1990). The Maintenance and Loss of Female and Male Signs in the Dublin Deaf Community. Michigan: UMI Dissertation Services.

LeMaster, B. (2000). Reappropriation of Gendered Irish Sign Language in One Family. Visual Anthropology Review, 15(2), 69-83. doi:10.1525/var.2000.15.2.69

LeMaster, B., \& Dwyer, J. P. (1991). Knowing \& using female \& male signs in Dublin. Sign Language Studies, 73, 361-396. 
Lewin, D., \& Schembri, A. (2011). Mouth gestures in British Sign Language: A case study of tongue protrusion in BSL narratives. Sign Language \& Linguistics, 14(1), 94-114. doi:10.1075/sll.14.1.06lew

Liddell, S. (1980). American Sign Language syntax. The Hague: Mouton.

Lucas, C. (1995). Sociolinguistics in deaf communities. Washington, D.C.: Gallaudet University Press.

Lucas, C. (Ed.). (2001). The sociolinguistics of sign languages. Cambridge: Cambridge University Press.

Lucas, C., Bayley, R., \& Valli, C. (2001). Sociolinguistic variation in American Sign Language (p. 237). Washington D.C.: Gallaudet University Press.

Lucas, C., \& Valli, C. (1992). Language contact in the American deaf community (p. 161). San Diego: Academic Press.

MacWhinney, B. (2000). The CHILDES Project: Tools for Analyzing Talk. Mahwah, NJ: Lawrence Erlbaum Associates.

Makoni, S., \& Pennycook, A. (2005). Disinventing and (Re)Constituting Languages. Critical Inquiry in Language Studies, 2(3), 137-156. doi:10.1207/s15427595cils0203_1

Makoni, S., \& Pennycook, A. (Eds.). (2006). Disinventing and reconstituting languages. Clevedon, UK: Multilingual Matters.

Masakata, N. (2003). From index-finger extension to index-finger pointing: Ontogenesis of pointing in preverbal infants. In S. Kita (Ed.), Pointing: Where Language, Culture and Cognition Meet (pp. 69-84). New Jersey: Lawrence Erlbaum Associates, Inc.

Massaro, D. W. (1998). Perceiving talking faces: From speech perception to a behavioral principle. Massachusetts: MIT Press.

McBurney, S. (2012). History of sign languages and sign language linguistics. In R. Pfau, M. Steinbach, \& B. Woll (Eds.), Sign Language. An international handbook (HSK 37, pp. 909-948). Berlin/Boston: De Gruyter Mouton.

McCarthy, M. (2002). Good listenership made plain: British and American non-minimal response tokens in everyday conversation. In R. Reppen, S. M. Fitzmaurice, \& D. Biber (Eds.), Using corpora to explore linguistic variation (pp. 49-71). Amsterdam/Philadelphia: John Benjamins.

McCarthy, M., \& O'Keeffe, A. (2010). What are corpora and how have they evolved? In A. O'Keeffe \& M. McCarthy (Eds.), The Routledge Handbook of Corpus Linguistics (pp. 3-13). New York: Routledge.

McCullough, S., \& Emmorey, K. (1996). Face Processing by Deaf ASL Signers: Evidence for Expertise in Distinguishing Local Features. Journal of Deaf Studies and Deaf Education, 2(4), 212-222.

Meier, R. P. (2000). Diminishing diversity of signed languages. Science, 288(5473), 1965-1965. doi:10.1126/science.288.5473.1965b

Mesch, J., Nilsson, A.-L., \& Wallin, L. (2011). Backchannel signals in signers' conversations. Gebärdensprachliche Textlinguistik: Stand Der Forschung Und Perspektiven. Göttingen.

Mitchell, R. E., \& Karchmer, M. A. (2004). Chasing the mythical ten percent: Parental hearing status of deaf and hard of hearing students in the United States. Sign Language Studies, 4(2), 138-163. doi:10.1353/sls.2004.0005 
Mitterer, H., \& McQueen, J. M. (2009). Processing reduced word-forms in speech perception using probabilistic knowledge about speech production. Journal of Experimental Psychology. Human Perception and Performance, 35(1), 244-63. doi:10.1037/a0012730

Mohammed, T., Campbell, R., MacSweeney, M., Barry, F., \& Coleman, M. (2006). Speechreading and its association with reading among deaf, hearing and dyslexic individuals. Clinical Linguistics \& Phonetics, 20(7-8), 621-630. doi:10.1080/02699200500266745

Mohr, S. (2011). Mouth Actions in Irish Sign Language - Their System and Functions. $\mathrm{PhD}$ dissertation. Universität zu Köln.

Mohr, S. (2012). The visual-gestural modality and beyond: Mouthings as a language contact phenomenon in Irish Sign Language. Sign Language \& Linguistics, 15(2), 185-211. doi: 10.1075/sll.15.2.01moh

Morford, J. P., Wilkinson, E., Villwock, A., Piñar, P., \& Kroll, J. F. (2011). When deaf signers read English: Do written words activate their sign translations? Cognition, 8, 286-292. doi:10.1016/j.cognition.2010.11.006

Muysken, P. (2000). Bilingual speech: A typology of code mixing. Cambridge: Cambridge University Press.

Muysken, P. (2013). Language contact outcomes as the result of bilingual optimization strategies. Bilingualism: Language and Cognition, 16, 709-730. doi:10.1017/S1366728912000727

Myers-Scotton, C. (2006). Multiple Voices. An Introduction to Bilingualism. Oxford: Blackwell publishing.

Mylander, C., \& Goldin-Meadow, S. (1991). Home sign systems in deaf children: The development of morphology without a conventional language model. In P. Siple \& S. D. Fischer (Eds.), Theoretical Issues in Sign Language Research, Volume 2: Psychology (pp. 41-63). Chicago: University of Chicago Press.

Nadolske, M. A., \& Rosenstock, R. (2007). Occurrence of mouthings in American Sign Language: A preliminary study. In P. Perniss, R. Pfau, \& M. Steinbach (Eds.), Visible Variation: Comparative Studies on Sign Language Structure (pp. 35-62). Berlin: Mouton De Gruyter.

Nedela, F. (2013). Solo Mouthings in Sign Language of the Netherlands. MA thesis. Radboud University Nijmegen.

Nespor, M., \& Sandler, W. (1999). Prosody in Israeli Sign Language. Language and Speech, 42(2-3), 143-176. doi:10.1177/00238309990420020201

Nonhebel, A., Crasborn, O., \& Van der Kooij, E. (2004). Sign language transcription conventions for the ECHO project. Retrieved from http://sign-lang.ruhosting.nl/echo/docs/ECHO_transcr_mouth.pdf

Nyst, V. A. S. (2007). A Descriptive Analysis of Adamorobe Sign Language (Ghana). Utrecht: LOT.

Nyst, V. A. S. (2012). Shared sign languages. In R. Pfau, M. Steinbach, \& B. Woll (Eds.), Sign Language. An international handbook (HSK 37, pp. 552-574). Berlin/Boston: De Gruyter Mouton.

Ormel, E., \& Crasborn, O. (2012). Prosodic Correlates of Sentences in Signed Languages: A Literature Review and Suggestions for New Types of Studies. Sign Language Studies, 12(2), 279-315. doi:10.1353/sls.2011.0019 
Ormel, E., Crasborn, O., \& De Meijer, A. (2013). Coarticulation of thumb position in Sign Language of the Netherlands. Paper presented at Theoretical Issues of Sign language Research, TISLR11, 10-13 July, London, UK. Retrieved from http://www.ucl.ac.uk/dcal/tislr/tislr-programme

Pfau, R. (2012). Manual communication systems: evolution and variation. In R. Pfau, M. Steinbach, \& B. Woll (Eds.), Sign Language. An international handbook (HSK 37, pp. 513-551). Berlin/Boston: De Gruyter Mouton.

Pfau, R., Steinbach, M., \& Woll, B. (Eds.). (2012). Sign Language. An international handbook (HSK 37). Berlin/Boston: De Gruyter Mouton.

Plaza-Pust, C. (2012). Deaf education and bilingualism. In R. Pfau, M. Steinbach, \& B. Woll (Eds.), Sign Language. An international handbook (HSK 37, pp. 949-979). Berlin/Boston: De Gruyter Mouton.

Rainò, P. (2001). Mouthings and mouth gestures in Finnish Sign Language (FinSL). In P. Boyes Braem \& R. Sutton-Spence (Eds.), The hands are the head of the mouth. The mouth as articulator in sign languages (pp. 41-50). Hamburg: Signum Press.

Rietveld-Van Wingerden, M., \& Tijsseling, C. (2010). Ontplooiing door communicatie. Geschiedenis van het onderwijs aan doven en slechthorenden in Nederland [Development through communication. History of the education of the deaf and hard of hearing in the Netherlands]. Antwerpen/Apeldoorn: Garant.

Roberts, L., \& Meyer, A. S. (2012). Individual Differences in Second Language Learning [Special Issue]. Language Learning, 62(Supplement S2). doi:10.1111/j.1467-9922.2012.00702.x

Sáfár, A. (in preparation). Handedness in deaf signers: the Corpus NGT and beyond. $\mathrm{PhD}$ dissertation. Radboud University Nijmegen.

Sáfár, A., \& Crasborn, O. (2013). A corpus-based approach to manual simultaneity. In L. Meurant, A. Sinte, M. Van Herreweghe, \& M. Vermeerbergen (Eds.), Sign language research, uses and practices. Crossing views on theoretical and applied sign language linguistics (pp. 179-203). Berlin/Boston: De Gruyter Mouton USA.

Sandler, W. (1999). Cliticization and prosodic words in a sign language. In T. A. Hall \& U. Kleinhenz (Eds.), Studies on the phonological word (Current Studies in Linguistic Theory) (pp. 223-254). Amsterdam: Benjamins.

Schembri, A. (2008). British Sign Language corpus project: Open access archives and the observer's paradox. In O. Crasborn, T. Hanke, E. Efthimiou, I. Zwitserlood, \& E. Thoutenhoofd (Eds.), Construction and exploitation of sign language corpora. Proceedings of the 3rd Workshop on the Representation and Processing of Sign Languages (LREC) (pp. 165-169). Paris: ELRA. Retrieved from http://www.lrec-conf.org/proceedings/lrec2008/workshops/W25_Proceedings.pdf

Schembri, A., \& Crasborn, O. (2010). Issues in creating annotation standards for sign language description. In Corpora and Sign Language Technologies. Proceedings of the 4th Workshop on the Representation and Processing of Sign Languages. Language Resources and Evaluation Conference (LREC) (pp. 212-216). Retrieved from http://www.sign-lang.uni-hamburg.de/lrec2010/lrec_cslt_01.pdf

Schembri, A., Fenlon, J., Rentelis, R., Reynolds, S., \& Cormier, K. (2013). Building the British Sign Language Corpus. Language Documentation and Conservation, 7, 136-154. 
Schembri, A., \& Johnston, T. (2007). Sociolinguistic Variation in the Use of Fingerspelling in Australian Sign Language: A Pilot Study. Sign Language Studies, 7(3), 319-347. doi:10.1353/sls.2007.0019

Scherer, K. R. (1986). Vocal affect expression: a review and a model for future research. Psychological Bulletin, 99(2), 143-165.

Scherer, K. R. (2003). Vocal communication of emotion: A review of research paradigms. Speech Communication, 40(1/2), 227-256. doi:10.1016/S0167-6393(02)00084-5

Schermer, T. (1985). Analysis of natural discourse of deaf adults in the Netherlands: Observations on Dutch Sign Language. In W. C. Stokoe \& V. Volterra (Eds.), SLR'83: Proceedings of the 3rd International Symposium on Sign Language Research (pp. 281-288). Silver Spring, MD: Linstok Press.

Schermer, T. (1990). In search of a language. Influences from spoken Dutch on Sign Language of the Netherlands. Delft: Eburon.

Schermer, T. (2003). From Variant to Standard: An Overview of the Standardization Process of the Lexicon of Sign Language of the Netherlands over Two Decades. Sign Language Studies, 3(4), 469-486. doi:10.1353/sls.2003.0017

Schermer, T., Koolhof, C., Muller, S., Geuze, J., \& Vink, M. (Eds.). (2009). Van Dale basiswoordenboek Nederlandse Gebarentaal [Basic dictionary of Sign Language of the Netherlands]. Utrecht and Antwerp: Van Dale.

Schuit, J. (2012). Signing in the Arctic: External influences on Inuit Sign Language. In U. Zeshan \& C. de Vos (Eds.), Sign Languages in Village Communities: Anthropological and Linguistic Insights (pp. 181-208). Boston/Berlin: Walter de Gruyter.

Scobbie, J. M., Punnoose, R., \& Khattab, G. (2013). Articulating five liquids: A single speaker ultrasound study of Malayalam. In L. Spreafico \& A. Vietti (Eds.), Rhotics: new data and perspectives (pp. 99-124). Bozen-Bolzano: Bozen-Bolzano University Press.

Senghas, A. (1995). Children's contribution to the birth of Nicaraguan Sign Language. $\mathrm{PhD}$ dissertation. Massachusetts Institute of Technology.

Siple, P. (1978). Visual constraints for sign language communication. Sign Language Studies, $19,95-110$.

Stadthaus, M. (2010). De mate van mondopening bij dove Nederlandse gebaarders [The degree of mouth opening in Dutch deaf signers]. MA thesis. Radboud University Nijmegen.

Stokoe, W. C. (1960). Sign language structure: an outline of the visual communication systems of the American deaf. Studies in Linguistics Occasional Paper 8. Re-issued 2005, Journal of Deaf Studies and Deaf Education, 10(1), 3-37. doi:10.1093/deafed/eni001

Sutton-Spence, R. (2007). Mouthings and simultaneity in British Sign Language. In M. Vermeerbergen, L. Leeson, \& O. Crasborn (Eds.), Simultaneity in signed languages (pp. 147-162). Amsterdam: John Benjamins.

Sutton-Spence, R., \& Day, L. (2001). Mouthings and mouth gestures in British Sign Language (BSL). In P. Boyes Braem \& R. Sutton-Spence (Eds.), The hands are the head of the mouth. The mouth as articulator in sign languages (pp. 69-86). Hamburg: Signum Press.

Sutton-Spence, R., \& Woll, B. (1999). The linguistics of British Sign language. An Introduction. New York: Cambridge University Press. 
Sze, F., Woodward, J., Wijaya, L. L., Satryawan, I., Isma, S., \& Suwiryo, A. I. (2013). Sign Language Use and Variations in Jakarta Sign Language. Poster presented at the Theoretical Issues in Sign Language Research Conference TISLR11, University College London, 10-13 July. London, U.K.

Terpstra, A., \& Schermer, T. (2006). Wat is NmG en waarom gebruik je het? [What is NmG and why use it?] Van Horen Zeggen, February 2, 10-17.

Tervoort, B. T. M. (1953). Structurele analyse van visueel taalgebruik binnen een groep dove kinderen [Structural analysis of visual language use within a group of deaf children]. Amsterdam: Noord-Hollandsche Uitgevers Maatschappij.

Van de Sande, I. (2009). Lexically bound mouth actions in Sign Language of the Netherlands: A comparison between two registers and two age groups. MA thesis. Radboud University Nijmegen.

Van de Sande, I., \& Crasborn, O. (2009). Lexically bound mouth actions in Sign Language of the Netherlands. A comparison between different registers and age groups. Linguistics in the Netherlands, 26, 78-90. doi:10.1075/avt.26.08san

Van den Bogaerde, B. (2000). Input and interaction in deaffamilies. Utrecht: LOT.

Van den Bogaerde, B., \& Baker, A. E. (2005). Code mixing in mother-child interaction in deaf families. Sign Language \& Linguistics, 8(1/2), 151-174. doi:10.1075/sll.8.1.08bog

Van der Kooij, E. (2002). Phonological Categories in Sign Language of the Netherlands: The Role of Phonetic Implementation and Iconicity. Utrecht: LOT.

Van der Zande, P. (2013). Hearing and seeing speech: Perceptual adjustments in auditory-visual processing. Nijmegen: Max Planck Institute for Psycholinguistics.

Van Heuven, W. J. B., \& Dijkstra, T. (2010). Language comprehension in the bilingual brain: fMRI and ERP support for psycholinguistic models. Brain Research Reviews, 64(1), 104-122. doi:10.1016/j.brainresrev.2010.03.002.

Van Veen, P. (2012). Verhaal halen bij doven. Een geschiedenis [Retrieving stories from the deaf. A history]. Deventer: Van Tricht uitgeverij.

Vermeerbergen, M., Leeson, L., \& Crasborn, O. (Eds.). (2007). Simultaneity in Signed Languages: Form and function. Amsterdam: John Benjamins.

Vinson, D. P., Thompson, R., Skinner, R., Fox, N., \& Vigliocco, G. (2010). The hands and mouth do not always slip together in British Sign Language: Dissociating articulatory channels in the lexicon. Psychological Science, 21(8), 1158-1167. doi:10.1177/0956797610377340

Vogt-Svendsen, M. (1981). Mouth Position \& Mouth Movement in Norwegian Sign Language. Sign Language Studies, 33(1), 363-376. doi:10.1353/sls.1981.0004

Vogt-Svendsen, M. (1983). Positions and movements of the mouth in Norwegian Sign Language (NSL). In J. Kyle \& B. Woll (Eds.), Language in sign: an international perspective on sign language (pp. 85-96). London: Croom Helm.

Vogt-Svendsen, M. (1984). Wordpictures in Norwegian Sign Language - a preliminary analysis. University of Trondheim Working Papers in Linguistics, 2, 112-141.

Vogt-Svendsen, M. (2001). A comparison of mouth gestures and mouthings in Norwegian Sign Language (NSL). In P. Boyes Braem \& R. Sutton-Spence (Eds.), The hands are the head of the mouth. The mouth as articulator in sign languages (pp. 9-40). Hamburg, Germany: Signum Press.

Wheatley, M., \& Pabsch, A. (2010). Sign Language Legislation in the European Union. Brussels: European Union of the Deaf. 
Wheatley, M., \& Pabsch, A. (2012). Sign Language Legislation in the European Union (2nd ed.). Brussels: European Union of the Deaf.

Wittenburg, P., Brugman, H., Russel, A., Klassmann, A., \& Sloetjes, H. (2006). ELAN: a professional framework for multimodality research. In Proceedings of the 5th International Conference on Language Resources and Evaluation (LREC 2006) (pp. 1556-1559). Genoa: Retrieved from http://www.lrec-conf.org/proceedings/lrec2006/pdf/153_pdf.pdf.

Woll, B. (2001). The sign that dares to speak its name: Echo phonology in British Sign Language (BSL). In P. Boyes Braem \& R. Sutton-Spence (Eds.), The hands are the head of the mouth. The mouth as articulator in sign languages (pp. 87-98). Hamburg: Signum Press.

Woll, B. (2012). Speechreading Revisited. Deafness \& Education International, 14(1), 16-21. doi:10.1179/1557069X12Y.0000000001

Woll, B., Sutton-Spence, R., \& Elton, F. (2001). Multilingualism and sign languages. In C. Lucas (Ed.), The sociolinguistics of sign languages (pp. 8-32). Cambridge: Cambridge University Press.

Wong, D., \& Peters, P. (2007). A study of backchannels in regional varieties of English, using corpus mark-up as the means of identification. International Journal of Corpus Linguistics, 12(4), 479-509. doi:10.1075/ijcl.12.4.03won

Zeshan, U. (2001). Mouthing in Indo-Pakistani Sign Language (IPSL): Regularities and variations. In P. Boyes Braem \& R. Sutton-Spence (Eds.), The hands are the head of the mouth. The mouth as articulator in sign languages (pp. 247-271). Hamburg: Signum Verlag.

Zeshan, U., \& De Vos, C. (Eds.). (2012). Sign languages in village communities. Anthropological and linguistic insights. Berlin: Mouton De Gruyter.

Zwitserlood, I. (2010). Sign language lexicography in the early 21st century and a recently published dictionary of Sign Language of the Netherlands. International Journal of Lexicography, 23(4), 443-476. doi:10.1093/ijl/ecq031 
142 | 


\section{Samenvatting in het Nederlands}

$\Phi$ it proefschrift gaat over de vraag hoe gesproken Nederlands en Nederlandse Gebarentaal (NGT) gecombineerd worden in het alledaags taalgebruik van Nederlandse doven. Hoewel vaak wordt gezegd dat doven met hun handen praten is er een belangrijke rol weggelegd voor andere manieren om de boodschap over te brengen. Zo kunnen opgetrokken wenkbrauwen een vraagintonatie aan de zin geven, speelt blikrichting een rol bij beurtwisseling, en is de mond op verschillende manieren actief. Mondbewegingen (mouth actions) kunnen worden verdeeld in twee groepen: mouth gestures (taaleigen mondgebaren, ook wel 'orale componenten' genoemd) en mouthings (uit de gesproken taal geleende woordbeelden, ook wel 'gesproken componenten' genoemd). Een voorbeeld van die eerste categorie, mouth gestures, is het bijvoeglijk gebruik van bolle wangen bij het gebaar BOEK, om zo aan te geven dat het een groot boek is, of het gebruik van een uitgestoken tongpunt bij een klein boek; een ander voorbeeld is het maken van getuite lippen bij het gebaar AANWEZIG, dat geen eigen betekenis heeft maar wel nodig is om het gebaar grammaticaal te maken. Ook kunnen mouth gestures deel uitmaken van complete 
gezichtsuitdrukkingen, zoals een open mond en grote ogen bij het gebaar VERBAZING. Dit proefschrift gaat echter niet over mouth gestures maar over mouthings, de (doorgaans geluidloos) uitgesproken Nederlandse woorden die dove gebaarders articuleren tijdens het gebaren. De titel van dit proefschrift ('de alomtegenwoordigheid van gesproken componenten in Nederlandse Gebarentaal') suggereert al dat mouthings (gesproken componenten) vaak voorkomen. Daar is al eerder onderzoek naar gedaan, maar meestal op basis van slechts enkele proefpersonen of informanten. Wat er gebeurt bij natuurlijk taalgebruik, wanneer doven vrijelijk met elkaar discussiëren, dat was nog niet eerder onderzocht aan de hand van grote hoeveelheden data. Hoe vaak worden mouthings nu eigenlijk gebruikt? Zit er variatie in het gebruik van de Nederlandse woorden die bij de gebaren worden gearticuleerd? Hoe verhouden mouthings zich tot de gebaren waarmee ze voorkomen? Dat wil zeggen, is er een één op één relatie tussen het gebaar en het woord, of zijn er andere combinaties mogelijk? Door het uitvoeren van vier verschillende onderzoeken in de relatief grote dataset van het Corpus NGT hebben we geprobeerd meer te weten te komen over de interactie tussen NGT en gesproken Nederlands. Deze onderzoeken en de resultaten ervan worden in de zes hoofdstukken van dit proefschrift beschreven. Samenvattingen in NGT zijn beschikbaar op www.gebareninzicht.nl. Hieronder volgt een Nederlandse samenvatting.

In hoofdstuk 1 wordt eerst een algemene inleiding gegeven over de geschiedenis van gebarentaal, het dovenonderwijs en gebarentaalonderzoek. Hoewel gebarentaal de meest toegankelijke taal is voor doven, heeft het gebruik ervan lange tijd onder druk gestaan. Op het dovenonderwijscongres van Milaan in 1880 werd besloten dat onderwijs aan doven erop gericht moest zijn om doven te leren spreken en te leren liplezen. Op veel dovenscholen werd het gebruik van gebarentaal helemaal uitgebannen, tot de slaapzalen aan toe. Mede onder invloed van het eerste gebarentaalwetenschappelijk onderzoek in de jaren vijftig en zestig van de vorige eeuw ontstond het besef dat het gebruik van gebarentaal helemaal nog niet zo'n gek idee was, en vanaf de jaren tachtig begint NGT langzamerhand weer zijn intrede te doen in het Nederlandse dovenonderwijs. De lange oralistische onderwijstraditie waar doven mee te maken hebben gehad heeft er waarschijnlijk mede voor gezorgd dat het gebruik van gesproken Nederlands zijn sporen heeft nagelaten in NGT, onder meer in de vorm van het gebruik van mouthings tijdens het gebaren (iets wat in hoofdstuk 4 verder aan bod komt). 
Over wat nu de linguïstische status is van deze mouthings wordt nog steeds discussie gevoerd. Hoewel vrijwel iedereen het er over eens is dat mouthings hun basis hebben in de omringende gesproken talen, verschillen onderzoekers met elkaar van mening over hoe mouthings geanalyseerd moeten worden. Sommigen zien mouthings als onderdeel van de gebarentaalstructuur (oftewel als onderdeel van het lexicon en van de morfosyntactische structuur van de gebarentaal), terwijl anderen het puur als een taalcontactfenomeen zien waarbij mouthings toevallig tegelijkertijd met gebaren voorkomen. Doordat gebarentalen nu eenmaal altijd in contact zijn met gesproken talen leidt dat tot het gebruik van elementen uit de gesproken taal in de gebarentaal, een proces dat bij twee gesproken talen code-mixing wordt genoemd; voor het mengen van gesproken en gebarentaal wordt wel de term code-blending gebruikt. Weer anderen verwerpen het idee van code-blending, omdat die term een pure vorm van gebarentaal veronderstelt die niet door gesproken taal beïnvloed is - iets wat in de praktijk niet voorkomt. In plaats daarvan worden mouthings als een van de componenten gezien van zogeheten composite utterances, samengestelde uitingen waarbij de mond slechts een van de gebruikte componenten is. De onderzoeken die wij hebben uitgevoerd en waarover we hieronder rapporteren lijken vooral voor codeblending te pleiten, hoewel er zeker iets te zeggen valt voor het idee van composite utterances.

Voor al het onderzoek dat we hier beschrijven is videomateriaal gebruikt uit het Corpus NGT. Dit corpus bestaat uit video-opnames van 92 dove moedertaalgebaarders die in paren zijn opgenomen en elkaar verhalen (na)vertellen en discussiëren over van tevoren vastgestelde onderwerpen, zoals het gebruik van gebarentaal, onderwijs en hoe het vroeger was om doof te zijn in vergelijking met tegenwoordig. Behalve dit videomateriaal zijn de annotatiebestanden een belangrijk bestanddeel van het corpus: tekstbestanden waarin per filmpje de gebruikte gebaren worden beschreven (betekenis, handvorm, beweging en dergelijke), evenals nietmanuele (maar wel linguïstische) activiteit zoals lichaamshouding, blikrichting, en bewegingen met de wenkbrauwen, neus en mond. Het annoteren van het corpus is een tijdrovend karwei dat handmatig moet gebeuren. Zes jaar na het afronden van de opnames van het corpus is minder dan een kwart door annotaties ontsloten. Automatische gebarenherkenning en -annotatie is weliswaar in ontwikkeling, maar bruikbare toepassing daarvan zal nog lange tijd op zich laten wachten.

In het Corpus NGT worden annotaties voor de mond op verschillende tiers (regels) gemaakt. Op de belangrijkste tier worden mouthings beschreven zoals ze 
worden waargenomen, dus met inachtneming van eventuele vervoeging of uitspraakreductie. Mouth gestures kunnen hier beschreven worden met behulp van bepaalde coderingen, maar dat is voor de onderzoeken in dit proefschrift niet gebeurd. In het geval van vervoeging of uitspraakreductie op de hoofdtier wordt op een aparte tier een annotatie gemaakt van de onvervoegde of ongereduceerde vorm. Op weer een andere tier wordt aangegeven of de hoofdannotatie een mouthing of een mouth gesture betreft. Ten slotte zijn er tiers voor het beschrijven van spreidende mouthings en toegevoegde mouthings, zoals gebruikt voor de studies beschreven in hoofdstukken 3 en 5 (zie hieronder). Voor iedere studie zijn weer nieuwe mondannotaties gemaakt, zodat elke volgende studie op een grotere set data gebaseerd kon worden en het corpus verder ontsloten werd. Het Corpus NGT is een van de uitgebreidste gebarentaalcorpora ter wereld, en is online te raadplegen; zie www.ru.nl/corpusngt/ voor meer informatie.

De eerste studie, beschreven in hoofdstuk 2, onderzoekt de mogelijke variatie binnen de Nederlandse woorden die als mouthing gebruikt worden. Zulke variatie kan een indicatie zijn voor de linguïstische status van mouthings: wanneer bij een gebaar altijd dezelfde mouthing voorkomt (wanneer bijvoorbeeld bij het gebaar KOFFIE altijd de mouthing 'koffie' gemaakt zou worden) kan dat duiden op een verankering van het mondbeeld aan het gebaar in het lexicon, en is de mouthing onderdeel van de fonologische specificatie van het gebaar geworden. Wanneer daarentegen veel variatie gevonden wordt (bijvoorbeeld als we bij KOFFIE de ene keer 'koffie' zouden vinden, maar de andere keer 'zwart' of 'lekker') suggereert dat juist dat er geen vaste relatie is tussen het gebaar en de mouthing, en zijn gebaarders creatief in het gebruik van twee talen tegelijkertijd, oftewel code-blending. Verder hebben we gekeken naar de frequentie en vorm van temporele reductie oftewel het inslikken van lettergrepen.

Om de mogelijke variatie te onderzoeken hebben we 20 van de meest voorkomende gebaren uit het Corpus NGT genomen en van ieder gebaar tussen de 20 en 100 tokens bekeken, van zoveel mogelijk verschillende personen. Bij twee gebaren, SCHOOL en BEGRIJPEN, vonden we geen variatie, maar alleen maar de mouthings 'school' en 'begrijp(en)', respectievelijk. Bij andere gebaren vonden we dat er weliswaar in de meeste gevallen dezelfde mouthing werd gebruikt, maar soms in plaats daarvan een mouth gesture. Er waren enkele gebaren, waaronder GROEP, waar we zowel variatie binnen mouthings vonden als variatie tussen mouthings en mouth gestures. In vrijwel alle gevallen van variatie binnen mouthings betrof het variatie 
binnen hetzelfde semantische veld, zoals 'goed', 'prima' of 'best' bij het gebaar GOED. Over het algemeen vonden we dat de meeste gebaren steeds met dezelfde mouthing voorkomen, ongeacht de woordklasse. Temporele reductie van mouthings komt veel voor; de lettergreep die in gesproken Nederlands beklemtoond is blijft altijd nog zichtbaar, maar er is veel variatie in de realisering van de overige lettergrepen. Deze twee vormen van variatie (die tussen mouthings en mouth gestures, en de verschillen in reductie) ondersteunen het idee dat mouthings een vorm van code-blending met gesproken taal zijn en dat ze niet zijn gespecificeerd in het lexicon.

De tweede studie, beschreven in hoofdstuk 3, beschrijft de spreiding van mouthings over naastliggende gebaren. Over het algemeen lijken gebaren en mouthings zo ongeveer tegelijkertijd te beginnen en te eindigen. Het komt echter regelmatig voor (en is ook op kleine schaal in ander onderzoek beschreven) dat er soms meerdere gebaren gemaakt worden in de tijd dat de mond één woord articuleert. Dit noemen we spreiding, omdat de mouthings zich uitbreiden (spreiden) vanuit een brongebaar. Onderzoek hiernaar is van belang omdat het ons iets kan vertellen over de interne structuur van gebarentaal: het laat ons zien op welke manier groepen van gebaren met elkaar verbonden kunnen worden.

Het sample dat we onderzocht hebben omvat 5929 mouthings. Het blijkt dat $14 \%$ daarvan spreidt over een of meer naastliggende gebaren. In de meeste gevallen (94\%) spreidt zo'n mouthing over het volgende gebaar; dan wordt bijvoorbeeld de mouthing 'school' gemaakt in de tijd dat de twee gebaren SCHOOL GAAN gearticuleerd worden. In dit geval heeft dat laatste gebaar (GAAN) dus geen eigen mouth action. In enkele gevallen (5\%) spreidt de mouthing over het voorafgaande gebaar, bijvoorbeeld wanneer de mouthing 'school' gemaakt wordt in de tijd dat de twee gebaren NAAR SCHOOL gearticuleerd worden. Ook kan een mouthing over een reeks van naastliggende gebaren spreiden (6\%). Spreiding over het voorafgaande gebaar of over meerdere gebaren komt dus niet heel veel voor, maar wordt wel door veel verschillende gebaarders gedaan - het zijn dus geen idiosyncratische verschijnselen. De meeste gevallen (58\%) van spreiding doen zich voor over wijsgebaren, in het Corpus NGT geannoteerd als PT (point). Mogelijk bakent de mouthing hiermee een kort prosodisch domein af en is dit een anwijzing dat wijsgebaren in sommige gevallen feitelijk clitics zijn geworden waardoor ze deel zijn gaan uitmaken van het naastliggende gebaar. 
De lengte van spreidende mouthings blijkt significant langer te zijn dan de lengte van niet-spreidende mouthings, zowel in millisecondes als in aantal lettergrepen. De vraag blijft echter of mouthings spreiden omdat de gebaren waar ze bij horen zo kort zijn of omdat de mouthings zelf zo lang zijn.

In de derde studie, beschreven in hoofdstuk 4, zijn we gaan kijken hoe vaak mouthings nu eigenlijk voorkomen in NGT, en of er verschillen te vinden zijn tussen mannen en vrouwen, Amsterdammers en Groningers (en Rotterdammers, Voorburgers en Sint-Michielsgestelaren - de plaatsen waar van oudsher dovenscholen gevestigd zijn), jongeren en ouderen, mensen met dove of horende ouders, hoog opgeleiden en laag opgeleiden. In vrijwel elke gesproken taal zijn verschillen te vinden langs een of meer van deze sociolinguïstische scheidslijnen. Voor gebarentaal geldt dat een verandering in het gebruik van mouthings te verwachten is, vanwege een verschuiving van zuiver spraakgericht onderwijs naar gebruik van meer gebarentaal op school. Voor Finse gebarentaal is dit eerder geopperd, en van Ierse gebarentaal is bekend dat er een verschil is in aantallen mouthings door mannen en vrouwen dat toegeschreven kan worden aan het feit dat het oralisme op de jongensinstituten op een later tijdstip is ingevoerd dan op de meisjesinstituten. Wanneer je het Corpus NGT doorbladert ontstaat de indruk dat er in NGT meer mouthings gemaakt worden door oudere gebaarders dan door jongere, hoewel in eerder (kleinschalig) onderzoek geen verschillen werden gevonden.

Ten tijde van deze studie was het aantal mondannotaties gegroeid tot ruim 10.000. Na een analyse van al deze annotaties bleek dat ruim $83 \%$ van alle mouth actions mouthings zijn: ze zijn werkelijk alomtegenwoordig. Hoewel er grote individuele verschillen waren, geldt voor alle gebaarders dat meer dan $65 \%$ van alle mouth actions tot de groep van mouthings behoren. Diezelfde grote individuele verschillen zouden er de oorzaak van kunnen zijn dat we geen verschillen hebben gevonden op basis van geslacht, regio, leeftijd, of het hebben van dove of horende ouders. Oftewel: frequentie van mouthings geeft geen indicatie van iemands sociale achtergrond. De enige uitzondering die we daarop vonden was het gebruik van mouthings bij de hoogst en de laagst opgeleiden: de hoogst opgeleide gebaarders gebruiken significant minder mouthings dan de laagst opgeleide. De verschillen zijn echter klein, en zelfs bij de hoogst opgeleiden (de groep met het kleinste aantal mouthings) bestaat $80 \%$ van de mouth actions nog altijd uit mouthings. We kunnen 
concluderen dat dove gebaarders van NGT bimodaal tweetalig zijn in elke gebareninteractie, ook wanneer er alleen maar doven aanwezig zijn.

De vierde studie, beschreven in hoofdstuk 5, beschrijft op welke manier losse mouthings voorkomen die niet met een gebaar samenvallen en analyseert hoe deze gebruikt worden om extra informatie aan een NGT-zin toe te voegen. We hebben hierbij drie verschillende typen mouthings onderscheiden die elk betekenis aan de uiting bijdragen zonder dat er een manueel equivalent gebruikt wordt: specificerende (specifying) mouthings, solo mouthings, en toegevoegde (added) mouthings.

Specificerende mouthings zijn mouthings die een betekenis aan het gebaar toevoegen of de betekenis van dat gebaar veranderen. Dat gebeurt bijvoorbeeld wanneer de mouthing 'moeilijk' het gebaar CONTACT vergezelt, zodat de betekenis 'moeilijk contact' wordt. Een ander voorbeeld is wanneer de mouthing 'altijd' tegelijk gearticuleerd wordt met het gebaar DISCUSSIEREN.

Solo mouthings zijn mouthings die gemaakt worden zonder dat de handen actief zijn, bijvoorbeeld wanneer deze in de schoot van de gebaarder liggen of wanneer de gebaarder met zijn of haar armen over elkaar zit. Veel van deze solo mouthings worden gemaakt als backchannel: een korte uiting als indicatie dat de geadresseerde het verhaal van de gesprekspartner volgt, zonder dat hij of zij de beurt wil overnemen, vaak door een kort ja of nee, of door de herhaling van een gebaar van de gebaarder door middel van een mouthing. Solo mouthings komen ook voor aan het begin of eind van een gebaarde zin: de gebaarder begint met een aantal mouthings zonder gebaren, en pas na een paar woorden komen de handen erbij.

Toegevoegde mouthings ten slotte zijn mouthings die kunnen voorkomen tijdens de transitie tussen twee gebaren (met elk hun eigen mouth action) of tijdens een gebaar dat al zijn eigen mouth action heeft. De meest voorkomende woordsoorten die als toegevoegde mouthing gebruikt worden zijn voegwoorden en werkwoorden, maar ook bijwoorden, voorzetsels en vraagwoorden komen veel voor, en het bijzondere is dat er geen woordklasse is die niet voorkomt.

Voor alle drie de types van mouthing geldt dat ze informatie overbrengen die ook met gebaren kunnen worden overgebracht. De gebaarders kiezen er in deze gevallen echter voor om gebruik te maken van de mogelijkheden die hun bimodaal tweetalige competentie hen biedt. We hebben hiermee laten zien dat mouthings niet louter één op één met een gebaar voorkomen, maar dat de grammaticale eigenschappen en betekenis van de twee talen gecombineerd kunnen worden tot 
samengestelde uitingen, die deels uit gebaren (met hun eigen mouthings en mouth gestures) en deels uit losse mouthings bestaan. Dat lijkt niet volgens een vast patroon te gebeuren; er is een continuüm van vermenging van NGT met gesproken Nederlands. Aan het ene uiteinde van dat continuüm vinden we NGT met standaard mouthings; langs het continuüm wordt de Nederlandse invloed steeds groter door middel van toegevoegde en solo mouthings, en nog verder wordt NGT afgewisseld met korte Nederlandse frases. NGT is hierbij de basistaal. Dit model vertoont grote overeenkomsten met een door andere onderzoekers voorgesteld model van NmG (Nederlands met Gebaren, een met gebaren ondersteunde vorm van gesproken Nederlands), dat beschrijft hoe NGT en gesproken Nederlands op verschillende manieren gecombineerd kunnen worden, maar dan met Nederlands als basistaal.

Hoofdstuk 6, ten slotte, vat de onderzoeken samen en bespreekt de waarde ervan voor taalkunding onderzoek in het algemeen en de gebarentaalwetenschap in het bijzonder. De algemene conclusie is dat mouthings een integraal onderdeel zijn van de dagelijkse communicatie tussen doven. Wat betreft de talige status van mouthings lijken al onze studies erop te wijzen dat er geen directe en vastliggende link is tussen de handen en de mond. Hoewel mouthing en gebaar weliswaar vaak dezelfde betekenis hebben vormen ze geen vaste combinatie. In hoofdstuk 2 vonden we variatie in het gebruik van mouthings tegenover mouth gestures en variatie in temporele reductie, in hoofdstuk 3 vonden we dat mouthings niet aan één enkel gebaar gekoppeld zijn, en in hoofdstuk 5 zagen we dat mouthings ook hun weg vinden in een gebarenzin zonder dat daar equivalente manuele activiteit tegenover hoeft te staan. We suggereren daarom dat mouthings niet gespecificeerd zijn in het NGT-lexicon, maar dat het gevallen van code-blending zijn waarvoor taalvaardigheid in twee talen benodigd is. Hoe het precieze mechanisme of de onderliggende processen achter deze vorm van code-mixing in elkaar zitten zal onderwerp moeten zijn van verder onderzoek.

In dit proefschrift hebben we laten zien dat de Nederlandse gesproken taal een grote invloed heeft op NGT. Mouthings uit het gesproken Nederlands zijn alomtegenwoordig. Vanwege deze invloed stellen we dat mouthings een prominentere plaats verdienen in gebarentaalonderzoek en dat ze zouden moeten worden opgenomen in iedere beschrijving van gebarencommunicatie. Hoewel we niet naar andere talen hebben gekeken, hebben we geen redenen om aan te nemen dat onze resultaten alleen voor de combinatie Nederlands/NGT gelden. Voor landen of 
gebieden met een vergelijkbare ontwikkeling van het dovenonderwijs en een vergelijkbare sociale positie van doven voorspellen we dat er vergelijkbare resultaten volgen uit een onderzoek als dit. Wij voorspellen dan ook dat onze bevindingen overdraagbaar zijn naar andere paren van gesproken taal en gebarentaal.

Tot besluit willen we er graag op wijzen dat in het najaar van 2014 de tweede uitgave van het Corpus NGT beschikbaar is gekomen (zie www.ru.nl/corpusngt/). In deze uitgave zijn ook de annotaties opgenomen die in het kader van dit project zijn gemaakt. Dit biedt onderzoekers over de hele wereld de mogelijkheid om ons onderzoek, onze data en onze conclusies te verifiëren dan wel te falsifiëren, om hun eigen observaties van andere gebarentalen te vergelijken met die van ons, en om de algemene kennis over de complexe interactie tussen gebaren en mouthings te vergroten. 
152 | 


\section{Curriculum vitae}

Richard Bank was born in 1969 in Amsterdam, the Netherlands. He started studying Sign Language Linguistics part-time at the University of Amsterdam in 2003, and obtained his Master's degree in General Linguistics in 2009, with specialisations in Phonetics and Sign Language Linguistics. In August 2009, he started his PhD research at the Centre for Language Studies at Radboud University Nijmegen.

As of June 2014, he works as a post-doctoral researcher in the NWO funded project Digging Into Signs, in collaboration with University College London. This project is aimed at creating standard annotation guidelines for sign language corpora annotation. 